\title{
Present Status of Precise Information on the Universal Physical Constants. Has the Time Arrived for Their Adoption to Replace Our Present Arbitrary Conventional Standards?*
}

\author{
JESSE W. M. DuMOND $\dagger$
}

\section{INTRODUCTION}

$\mathrm{T}$ THREE years ago Dr. E. R. Cohen and I prepared and published our latest (1955) least-squares adjustment of all the most reliable data then available bearing on the universal constants of physics and chemistry. Since then new data and information have been accumulating so that a year or two from now the time may perhaps be propitious for us to prepare a new adjustment taking the newly-gained knowledge into account. At present it is too early to attempt such a re-evaluation since many of the investigations and redeterminations now under way are still far from completed. I shall be obliged, therefore, to content myself in this talk with a description of the sources of information upon which our 1955 evaluation was based, mentioning however, the weak points where these are now either well established as errors or at least considered to be under strong suspicion of systematic error. I shall also tell you a little of some of the new re-evaluations now under way.

The experimental data upon which Cohen and I have based our (1955) evaluation for the universal physical constants fall into two groups. In the group I shall discuss first, seven precisely-measured experimental data are so interrelated that they overdetermine four primary unknowns. This situation requires us to arrive at the output results by a least-squares adjustment. On the other hand, the auxiliary constants, which constitute the second group, are quantities so precisely known relative to the accuracy of the measurements on the first group, and are involved with the latter in the least-squares adjustment in such a fashion, that nothing is gained by treating them as adjustable unknowns. Their measured values are, therefore, not subject to adjustment and are treated as though they were exactly known constants.

The four primary unknowns which we selected for the least-squares adjustment are the Sommerfeld fine structure constant, $\alpha$, the electronic charge, $e$, the Avogadro number, $N$, and the conversion constant $\lambda_{g} / \lambda_{s}$ (which we shall abbreviate as capital $\Lambda$ ) relating the two scales of wavelength, in angstroms on the one hand and in kilo $\mathrm{X}$ units (Siegbahn) on the other. It is characteristic of

* Manuscript received by the PGI, August 1, 1958.

$\uparrow$ California Institute of Technology, Pasadena, Calif. this subject that the experimentally measured data of ten do not give the desired unknowns directly but instead give functions of the unknowns.

Seven different functions of these above four unknowns have been measured by experimental methods which we feel are sufficiently precise and reliable to qualify them as input data in a least-squares adjustment. These seven experimentally determined numerical values are not only functions of the unknowns, $\alpha$, $e, N$, and $\Lambda$, but also of the above-mentioned experimentally determined auxiliary constants, of which five different kinds are listed in Table I. Another of these auxiliary constants I find it expedient to recall to your attention at the very beginning to avoid any possibility of confusion. This is the conversion factor, $r$, between the "physical" and "chemical" scales of atomic weight.

\section{The Chemical and Physical Scales of Atomic Weights}

The older of these two, the chemical scale, had its origin before the existence of isotopes was known. On the chemical scale the mean atomic weight of a mixture of the three isotopes of oxygen, $\mathrm{O}^{16}, \mathrm{O}^{17}$, and $\mathrm{O}^{18}$, "in their naturally occurring abundance ratios," is defined as having atomic weight 16.0000 . On the physical scale the atomic weight of the $\mathrm{O}^{16}$ isotope has, by definition, the atomic weight exactly 16 . Atomic weights on the physical scale are numerically slightly the larger.

On the basis of the following assumed isotopic abundance ratio

$$
\mathrm{O}^{16}: \mathrm{O}^{18}: \mathrm{O}^{17}=(506 \pm 10): 1:(0.204 \pm 0.008),
$$

and the masses $\mathrm{O}^{16}=16.0000$ (by definition), $\mathrm{O}^{18}$ $=18.0049$, and $\mathrm{O}^{17}=17.0045$. Birge [1] has computed the conversion factor, $r$, between the chemical and physical scales of atomic weight as

$$
r=1.000272 \pm 0.000005 \text { (Birge, 1941). }
$$

However, the definition of the chemical scale of atomic weights is an equivocal one because there is no exactly defined "naturally occurring abundance ratio" of the oxygen isotopes. Nier in a study [2] of the relative abundance of isotopes would revise Birge's value of $r$ upward about 6 parts per million, if the abundance ratio for atmospheric oxygen is taken as defining the chemical scale of atomic weights. Nier says that oxygen from 
TABLE I

Four Primary Unknowns Whose Least-Squares Adjusted Values Are Sought

\begin{tabular}{|c|c|c|c|c|}
\hline \multicolumn{4}{|c|}{$\begin{array}{l}\alpha\left(=2 \pi e^{2} h^{-1} c^{-1}\right. \\
e \\
N \\
\Lambda\left(=\lambda_{g} / \lambda_{s}\right)\end{array}$} & $\begin{array}{l}\text { ield's fine structure constant } \\
\text { ic charge (expressed in abs. esu) } \\
\text { o number } \\
\text { on factor from X units (Siegbahn) to milliangstroms }\end{array}$ \\
\hline \multicolumn{5}{|c|}{ First Group (Least-squares adjustment)-Seven Kinds of Experimental Data Bearing on Above Unknowns } \\
\hline $\begin{array}{l}\text { Kind } \\
\text { No. }\end{array}$ & Function & Measured Numeric & $\begin{array}{l}\text { Estimated } \\
\text { Precision }\end{array}$ & Description of Experiment \\
\hline 1 & \multicolumn{2}{|c|}{$\begin{array}{l}\mathrm{Ne} / \mathrm{c}=9652.15 \\
\mathrm{Ne} / \mathrm{c}=9651.29\end{array}$} & $\begin{array}{l}(13 \mathrm{ppm}) \\
(20 \mathrm{ppm})\end{array}$ & $\left.\begin{array}{l}\text { Iodine } \\
\text { Silver }\end{array}\right\}$ Faraday by electrochemistry \\
\hline $\begin{array}{l}2 \\
3 \\
4\end{array}$ & \multicolumn{2}{|c|}{$\begin{aligned} \Lambda & =1.002020 \\
N \Lambda^{3} & =0.606179 \times 10^{24} \\
\alpha^{3} c / e & =2.425517 \times 10^{13}\end{aligned}$} & $\begin{array}{l}(30 \mathrm{ppm}) \\
(38 \mathrm{ppm}) \\
(23 \mathrm{ppm})\end{array}$ & $\begin{array}{l}\text { X-ray lines measured with ruled gratings } \\
\text { Crystal densities and grating constants in X units } \\
\text { Proton Gyromagnetic Ratio (Thomas, Driscoll, and Hipple) }\end{array}$ \\
\hline 5 & \multicolumn{2}{|c|}{$\begin{array}{l}N e^{2} /\left(\alpha^{3} c^{2}\right)=3.979879 \times 10^{-10} \\
N e^{2} /\left(\alpha^{3} c^{2}\right)=3.979423 \times 10^{-10} \\
N e^{2} /\left(\alpha^{3} c^{2}\right)=3.979359 \times 10^{-10} \\
N e^{2} /\left(\alpha^{3} c^{2}\right)=3.979444 \times 10^{-10}\end{array}$} & $\begin{array}{l}(36 \mathrm{ppm}) \\
(11 \mathrm{ppm}) \\
(14 \mathrm{ppm}) \\
(36 \mathrm{ppm})\end{array}$ & $\left.\begin{array}{l}\text { Bloch and Jeffries, "Inverse cyclotron" } \\
\text { Sommer, Thomas, and Hipple, "Omegatron" } \\
\text { Collington, Dellis, Sanders, and Turberfield } \\
\text { Trigger's correction to Bloch and Jeffries }\end{array}\right\} \begin{array}{l}\text { Proton magnetic moment } \\
\text { in nuclear magnetons by } \\
\text { measuring } \omega_{s} / \omega_{p}\end{array}$ \\
\hline 6 & \multicolumn{2}{|c|}{$\alpha^{2} c=1.596412 \times 10^{6}$} & $(9 \mathrm{ppm})$ & $\left\{\begin{array}{l}\text { Microwave determination of fine } \\
\text { structure splitting in deuterium }\end{array}\right\}$ Lamb and coworkers \\
\hline 7 & \multicolumn{2}{|c|}{$\begin{array}{l}e c /(\Lambda \alpha)=1968.750 \\
e c /(\Lambda \alpha)=1968.911 \\
e c /(\Lambda \alpha)=1968.869\end{array}$} & $\begin{array}{l}(51 \mathrm{ppm}) \\
(40 \mathrm{ppm}) \\
(83 \mathrm{ppm})\end{array}$ & $\left.\begin{array}{l}\text { Felt, Harris, and DuMond } \\
\text { Bearden, Johnson, and Watts } \\
\text { Bearden and Schwarz }\end{array}\right\}$ Short wavelength limit of continuous \\
\hline \multicolumn{5}{|c|}{ Second Group (Regarded as fixed constants) } \\
\hline \multicolumn{5}{|c|}{$\begin{aligned} H & =1.008142 \pm 0.000003(\text { Physical })(3 \mathrm{ppm}) \\
H / M_{p} & =1.00054461 \\
D & =2.014735 \pm 0.000006(\text { Physical })(3 \mathrm{ppm}) \\
D / M_{d} & =1.00027244\end{aligned}$} \\
\hline \multicolumn{3}{|c|}{$\begin{aligned} R_{\infty} & =109737.309 \pm 0.012 \mathrm{~cm}^{-1} \\
\mu_{e} / \mu_{p}^{\prime} & =658.2288 \pm 0.0004 \\
\mu_{e} / \mu_{0} & =1.00114536 \\
c & =299793.0 \pm 0.3 \mathrm{~km}^{-1}\end{aligned}$} & $\begin{array}{l}\text { Spectro } \\
\text { m) Ratio e } \\
\text { m) Ratio e } \\
\text { m) Velocity }\end{array}$ & $\begin{array}{l}\text { determinations of } R_{\infty} \\
\text { a magnetic moment to proton magnetic moment } \\
\text { a magnetic moment to Bohr magneton }\end{array}$ \\
\hline
\end{tabular}

limestone would agree with this but if oxygen from iron ore or water were employed, the $\mathrm{O}^{18}$ content might be four per cent lower and the conversion factor, $r$, would be only about 1.000268 , some $4 \mathrm{ppm}$ lower than that chosen by Birge. Several proposals are now under discussion for redefinition and/or unification of these

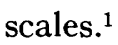

Because of the unequivocal nature of its definition and because extremely accurate atomic weights can be derived in terms of it from measurements of nuclear reaction energies, at present we shall base our calculations on the physical scale of atomic weights.

${ }^{1}$ In a private communication from E. Wichers, U. S. National Bureau of Standards, we are informed that the International Commission on Atomic Weights has been considering redefinition of the chemical scale of atomic weights. The definition they proposed would be based on the use of the exact number, 16, as the atomic weight of a mixture of the natural isotopes of oxygen whose average atomic mass is greater by an exact factor (probably 1.000275) than the mass of $\mathrm{O}^{16}$. A proposal strongly urged by Prof. J. Mattauch of Mainz, Ger., which now seems more likely to be adopted by both chemists and physicists, is to define a brand new scale of atomic weights and nuclidic masses such that on it the isotope ${ }^{12} \mathrm{C}$ would have exactly the value 12 . On this new scale the numerical values of all the present physical atomic weights would have to be revised but they could be expressed with better precision than at present for good operational reasons. A further advantage of the new ${ }^{12} \mathrm{C}=12$ scale would be that on it the numerical values of the mean atomic weights of natural mixtures of isotopic elements would not differ from the present chemical scale of atomic weights by amounts significant for the precision attainable by chemical methods.

\section{PART I}

\section{Input Data for Least Squares Adjustment}

\section{Faraday Constant by Electrochemical Methods}

The silver [3] and the iodine [4], [5] coulometers are the two instruments for measuring the Faraday which have received the most study. The experimental work was done in both cases prior to 1916 before the existence of isotopes was known! A study of the work on the silver voltameter clearly shows that the emphasis at that time was directed towards obtaining reproducible values of the electrochemical equivalent rather than a measurement of absolute fundamental significance for the purpose of determining the Faraday. This was natural since the objective at the time was to obtain specifications for a working procedure which would define empirically the ampere with as much reproducibility as possible. Four possible sources of error in the use of the silver voltameter for an absolute precision determination of the Faraday can be cited. 1) Not all of the current measured electrically may have been transported by the ions. 2) The measured gain in weight of the cathode may not be entirely due to deposited silver; there may be in the deposit "inclusions" of other matter from the electrolyte. 3) Some of the deposited silver may redissolve in the electrolyte, or otherwise become detached, 
before weighing. 4) The deposited silver may not have quite the same isotopic constitution as that of "natural" silver on which the accepted value of the chemical atomic weight of that element (the value used in computing the Faraday) is based. When these voltameter experiments were performed isotopes had not yet been discovered, and the atomic weight of a naturally occurring element such as silver was thought to be an invariable quantity. It is now known that electrolysis may well introduce an appreciable change in isotopic constitution and thereby affect the mean atomic weight. Silver has two isotopes of almost equal abundance which differ in mass by about two per cent. This abundance ratio is the most favorable one for producing a systematic error through selective electrolytic separation. If in the cathode deposit the ratio of the light to the heavy isotope content is greater by one per cent than it is in the solution, the deposit will be $46 \mathrm{ppm}$ lighter than if no such selective separation had occurred.

Iodine, on the other hand, occurs naturally in only one stable isotopic form, $\mathrm{I}^{127}$, and it is possibly significant that the electrolytic Faraday determinations of Vinal and Bates [4], [5] made with the "iodine coulometer" disagree with those of the silver voltameter by an amount considerably greater than the obvious experimental estimates of uncertainty would lead one to expect. Not only is this true but the iodine results are in much better accord with the consensus of present data on the atomic constants than are the silver results.

In the iodine coulometer the reaction

$$
\mathrm{I}_{3}^{-}+2(-)=3 \mathrm{I}^{-}
$$

takes place from left to right at the cathode and in the reverse sense at the anode. The changes in the amounts of "free iodine" at the two electrodes are determined by titration. Their equality (with opposite sign) furnishes an important check on the purity of the reaction. The iodine coulometer therefore contains within itself the proof of its own correctness. ${ }^{2}$

2 This instrument was first developed by E. W. Washburn and further perfected by one of his students, S. J. Bates. In view of the great advantages of the iodine coulometer over the silver voltameter it is most surprising that this method was not adopted in place of the electrolysis of silver for standardizing and reproducing the coulomb and the ampere by the International Technical Committee in the early part of this century. These advantages, listed by Bates in his $\mathrm{Ph}$.D. dissertation, 1912 [4] were later substantiated fully by a study at the U. S. National Bureau of Standards done in 1914 by Bates and Vinal [5]. Bates in his dissertation explains the working of the iodine coulometer as: "An aqueous solution of potassium iodide to which iodine has been added contains both iodide and tri-iodide ions. When a current of electricity is passed through such a solution the reaction $\mathrm{I}_{3}{ }^{-}+2(-)=3 \mathrm{I}^{-}$takes place in the direction from left to right at the cathode and in the reverse direction at the anode. Hence a cell in which the electrodes are surrounded by such a solution should contain the same total amount of 'free iodine' before and after the passage of an electric current. By separating the electrodes and the solutions surrounding them by a conducting solution of an iodide, the changes in the amounts of 'free iodine' at the two electrodes can be determined by titration and if the reaction is a perfectly clean-cut one, free from all disturbing side reactions, the results at the two electrodes should check each other. The iodine coulometer, therefore, contains within itself the proof of its own correctness, for if it can be shown that identical amounts of iodine are involved in the reactions at both electrodes under varying conditions of concentration and
The Faraday values obtained by the two coulometers have recently been recalculated by Vinal (1949) and later (1951) corrected by Hipple for the new atomic mass of iodine with the following results:

Iodine Faraday $=\mathrm{Ne}^{\prime}=9652.15$

$$
\pm 0.13 \text { abs emu (g equiv) }{ }^{-1} \text { (physical scale), }
$$

or as recalculated by Craig and Hoffman in the NBS booklet "Electrochemical Constants"

$$
\begin{aligned}
\text { Iodine Faraday } & =\mathrm{Ne} / \mathrm{c} \\
& =9652.5 \mathrm{emu}(\mathrm{g} \text { mole })^{-1}(\text { physical })
\end{aligned}
$$

Silver Faraday $=\mathrm{Ne}^{\prime}=9651.29$

$$
\pm 0.19 \text { abs emu (g equiv) })^{-1} \text { (physical scale). }
$$

The difference between these two results, $0.86 \pm 0.23$ emu (g mole $)^{-1}$ (physical) is uncomfortably large in comparison to its expected standard error.

It was the effort to achieve better consistency in the entire picture of the determinations bearing on the atomic constants which first emphasized the above discrepancies $^{3}$ and indicated that the iodine value was more likely to be the true one.

Our most recent adjustment of the atomic constants still supports the view that the iodine value is more nearly correct than the silver value as our subsequent analysis will show. The true source of the discrepancy is still obscure, however, in spite of two further investigations $s^{4}$ undertaken in an effort to clarify the matter.

current density, it will establish beyond question the fact that the only reaction, involving electricity, which occurs at the anode, is that expressed by the above equation. In this particular the iodine coulometer possesses an enormous advantage over the silver coulometer, for, as is well known, no such quantitative proof of the nature of the reaction is possible with the latter coulometer." Bates compared the results of eight runs with two iodine coulometers in series and obtained an average deviation from the mean of each pair of results of only $20 \mathrm{ppm}$.

3 As early as 1928 Birge [6] distinguished a systematic difference between what he called the "spectroscopic" and the "deflection" values of $e / m$, the charge-to-mass ratio for the electron. He at first thought that $e / m$ might be different depending on whether the electron was "inside" an atom or "in free space." In reality his so-called spectroscopic measurements of $e / m$ amounted to determinations of the ratio of the electronic and nuclear masses (by comparison of the Rydberg values for different light atoms) and a value of the Faraday constant had to be introduced to compute $e / m$ from the spectroscopic data. This constant did not enter in deriving $e / m$ from "deflection" measurements, however. Birge initially had preferred the silver value to the iodine value because of the great care which had been expended to make the method highly reproducible and partly because it was then regarded as the basis for defining the international coulomb. DuMond first pointed out (in 1940) that the apparent difference between the two sets of values of $e / m$ might stem from a systematic error in the silver Faraday value, a suggestion which Birge [7] immediately accepted as plausible.

${ }^{4}$ One of these was by Scott, Reed College, Portland, Ore. [8], and the other by Craig and Hoffman, U. S. National Bureau of Standards [9]. The first comprised a careful review of all methods to determine the amount of "inclusions" in the silver deposit of the silver coulometer and an attempt to estimate inclusions by means of radioactive tracers. The second was a redetermination of the Faraday with a coulometer employing the electrolytic oxidation of sodium oxalate in a supporting solution of sulphuric acid. The reaction, $\mathrm{C}_{2} \mathrm{O}_{4}^{--}$ $\rightarrow 2 \mathrm{CO}_{2}+2(-)$ was carried out in a coulometer with a gold anode and a platinum cathode, the anode and cathode vessels being connected electrically through four siphons dipping into three beakers to isolate anode and cathode solutions. A weighed quantity of sodium oxalate was added to the anode vessel and, after the electrolytic 
Further work on the iodine coulometer is reported to be in promising progress at the Rockefeller Institute for Medical Research, New York, N. Y., by MacInnes [10], using several improvements depending on newly developed modern techniques. The high Faraday value obtained with the iodine coulometer has received support also from a direct measurement of the charge-tomass ratio for protons of which I shall speak presently.

\section{The Conversion Factor, $\Lambda=\lambda_{g} / \lambda_{s}$, from Siegbahn $X$ Units to Milliangstroms}

The wavelengths of $\mathrm{X}$-ray spectral lines measured relative to each other by the high precision methods of crystal diffraction are known with a precision of one part in $10^{4}$ to $10^{5}$. This precision exploration and tabulation of the X-ray spectrum was first brought to high perfection by Siegbahn [11] and his school. Later, with such improvements as the two-crystal spectrometer and the curved crystal spectrometer, it was carried even further by many others [12], [13]. About 3000 of these precision wavelength measurements have been tabulated [14] and they constitute a very reproducible and well-defined natural scale of lengths in the range between about $10^{-9}$ and $4 \times 10^{-6} \mathrm{~cm}$. To express these wavelengths in centimeters or angstrom units the method first adopted was that of Bragg [15] in which the Avogadro number was taken as the quotient of the Faraday by Millikan's oil drop value of $e$. From the Avogadro number and the atomic weights of its constituents the absolute weight in grams of the unit cell of a crystal could be computed and this together with the macroscopic density and geometry of the crystal permitted calculation of its grating constant. As the result of precision work by Compton, Beets, and DeFoe [16] on calcite, Siegbahn adopted as the basis for calculating X-ray wavelengths the "effective"5 grating

oxidation, the residual oxalate was determined by titration with $\mathrm{KMnO}_{4}$. As Craig and Hoffman point out, the oxalate coulometer has the advantage that since a large fraction by weight of the measured substance, $\mathrm{C}_{2} \mathrm{O}_{4}$, is oxygen, the errors resulting from uncertainties in atomic weight are minimized. Seventeen determinations of the Faraday, twelve with a small and five with a large oxalate coulometer, were made, with results ranging from about 9652.6 to 9651.1 absolute emu per gram equivalent on the physical scale of atomic weights. The mean of their oxalate values came out lower than the iodine Faraday. The difference, $0.8 \pm 0.32$ abs emu (g equiv) ${ }^{-1}$, is still uncomfortably large relative to its expected standard deviation. The difference between the mean of the oxalate values and the silver value on the other hand is $0.06 \pm 0.36$ abs emu (g equiv) $)^{-1}$. This very good agreement could indeed be considered as a verification of the silver value if no other information were available. Actually, however, a study of the sequence of results obtained in seventeen measurements by the oxalate method shows a decidedly marked trend downward when the results are arranged in the temporal order in which the measurements were performed. The first two measurements are in good accord with the weighted mean of the iodine values whereas the mean of the last two measurements yields a value more than one absolute emu lower. This downward trend shows no suggestion of leveling off in the plot presented in Craig and Hoffman's paper. Such behavior strongly suggests an experiment "out of control." The reason is obscure but the suspicion is inescapable.

5 The "effective" grating space, $d_{n}$, includes a certain correction term for refractive index and is not strictly a constant but a function of the order of interference in Bragg reflection. For the calcite cleavage planes, for example, $d_{n}$ is related to the true grating space, $d$, by the equation, $d_{n}=d\left[1-135 \times 10^{-6} / n^{2}\right]$, and the corrected Bragg equation is written $n \lambda=2 d_{n} \sin \theta_{n}$. space of calcite at $18^{\circ} \mathrm{C}$ in first-order reflection at the conventional value

$$
d_{18}{ }^{\prime \prime}=3029.040 \mathrm{X} \text { units. }
$$

Siegbahn's intention was that the $\mathrm{X}$ unit should be one milliangstrom. He very wisely, however, chose to call this unit of length by a distinctive name, no doubt because he realized that its absolute value might some day require revision. We now know that Millikan's oil drop value of $e$ was erroneous chiefly because of the erroneous value of the viscosity of air which entered his computations, and this error was propagated into the calculations which led to the calcite grating space. For this reason it is best to regard Siegbahn's system of wavelengths in $\mathrm{X}$ units as a purely arbitrary unit system.

The discrepancy between Siegbahn's X unit and the milliangstrom was first noted by Bäcklin [17], I believe, when the wavelengths of certain soft $\mathrm{X}$-ray lines, which had been measured in $\mathrm{X}$ units by the methods of crystal diffraction, were also precisely measured with artificially ruled gratings calibrated as to grating constant with optically known wavelengths.

Save for the case of extremely long X-ray wavelengths. the great bulk of the ruled grating measurements of $\mathrm{X}$-ray lines have been made using plane gratings in grazing incidence. Compton and Doan [18] and shortly afterward Thibaud [19] were the first to use this method. Fig. 1 illustrates the geometry for the case of grazing incidence and compares the dispersions obtainable in grazing and in normal incidence. A condition for sufficient intensity in the diffracted spectrum is that the angle of grazing incidence shall be less than the critical angle for total reflection of the $\mathrm{X}$ rays.

The outstanding published experimental work on the comparison of grating and Siegbahn wavelengths of $\mathrm{X}$-ray lines has been done by Bearden [20], Bäcklin [21], Södermann [22], and by Tyrén [23]. The results have been summarized by Birge [24] in 1945 and are shown in Table II, together with the weighted average value of the conversion factor, $\Lambda$, which he adopted at that time,

$$
\Lambda=1.002030 \pm 0.000020 .
$$

In 1947, however, Bragg, after consultation with Siegbahn, Warren, and Lipson, and with due consideration given to the above data listed by Birge, recommended for general adoption

$$
\Lambda=1.002020 \pm 0.000030 .
$$

The chief argument for this revision downward in 1947 was based on the work of Tyrén in his dissertation (Uppsala, 1940). In this work, by means of a concave grating vacuum spectrometer, Tyrén had carefully compared on the same plates the wavelength positions of four X-ray lines with the wavelength positions of several Lyman series spark lines emitted by highly ionized oneelectron atoms. The wavelengths of these calibration 
Ruled gratings calibrated with optical light are used

at grazing incidence to measure $x$-ray line wavelengths.

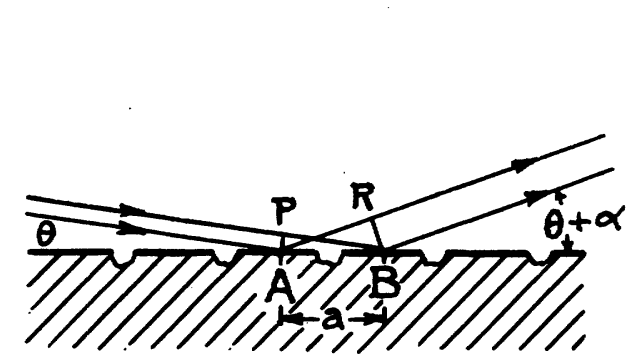

Path difference,

$\begin{aligned} \mathrm{PB}-\mathrm{AR} & =a[\cos \theta-\cos (\theta+a)] \\ & =n \lambda\end{aligned}$

In the simplest case of completely grazing incidence we have:

$\theta \rightarrow 0$ and $n \lambda=a(1-\cos a)=a a^{2} / 2$

DISPERSION FOR NORMAL AND FOR GRAZING INCIDENCE COMPARED

Grazing Incidence:--

$$
(d \alpha / d \lambda)_{g}=\sqrt{\frac{n}{2 a \lambda}}
$$

If $\lambda=1 \mathrm{~A}, \mathrm{a}=10^{-4}$ an and $n=I$,

$(d \alpha / d \lambda)_{g}=0.4 \cdot 10^{-2} \mathrm{radians} / \mathrm{A}$
Normal Incidence:-

$(d \alpha / d \lambda)_{n} \cong n / a($ for small $a)$

If $a=10^{-4}$ an and $n=1$

$(\mathrm{d} \alpha / \mathrm{d} \lambda)=10^{4} \mathrm{radians} / \mathrm{A}$

Fig. 1-Conversion factor, $\Lambda=\lambda_{g} / \lambda_{s}$, from X units to milliangstroms by X-ray diffraction on ruled gratings.

TABLE II

Birge's Comparison of Grating and Siegbahn WAVELENGTHS OF X RAYS (1945)

\begin{tabular}{l|c|c|c}
\hline \hline Author & X-Ray Line & $\begin{array}{c}\text { Value of }(\Lambda-1) \times 10^{6} \\
\text { PE Based on Accidental } \\
\text { Errors Only }\end{array}$ & $\begin{array}{c}\text { Birge's } \\
\text { Adopted PE }\end{array}$ \\
\hline Bearden & CuK $\alpha_{1,2}$ & $1980 \pm 31$ & \pm 52 \\
Bearden & CuK $\beta_{1,3}$ & $2079 \pm 28$ & \pm 50 \\
Bearden & CrKK $\alpha_{1,2}$ & $2036 \pm 21$ & \pm 48 \\
Bearden & CrK $\beta_{1,3}$ & $2017 \pm 28$ & \pm 48 \\
Söderman & AlK $\alpha_{1,2}$ & $2070 \pm 37$ & \pm 84 \\
Bäcklin & AlK $\alpha_{1,2}$ & $2000 \pm 14$ & \pm 60 \\
Bearden & CuK $\alpha_{1,2}$ & $2087 \pm 55$ & \pm 130 \\
Tyrén & AlK $\alpha_{1,2}$ & $2024 \pm 5$ & \pm 48 \\
\hline
\end{tabular}

Birge's 1945 adopted average: $\Lambda=1.002030 \pm 0.000020$.

Bragg (1947) after consultation with Siegbahn, Warren, and Lipson, with due consideration given to the above data listed by Birge, recommended for general adoption $\Lambda=1.002020 \pm 0.000030$.

lines he calculated using formulas based on the Sommerfeld-Dirac theory. At that time that theory was considered to give a rigorously accurate description of the one-electron atom. We now know that the formula used by Tyrén requires a correction for the "Lamb shift" varying from $100 \mathrm{ppm}$ for his $\mathrm{O}^{\text {virI }}$ Lyman alpha line to about $27 \mathrm{ppm}$ for his $\mathrm{Be}^{\mathrm{IV}}$ Lyman delta line. Fig. 2 shows graphically the relative amount, in parts per million, by which the calibration wavelengths used by Tyrén must be increased to correct them for the Lamb shift, and the values are tabulated numerically in Table III. Tyrén's original uncorrected value was $\Lambda=1.00199$.

The need for this correction went unnoticed until June, 1956, when Cohen and I were the first to call attention to it. Unfortunately, the dissertation of Tyrén does not give the precise way in which his different calibration lines were associated in their different orders of reflection with his different $x$-ray lines,' so that it is im-

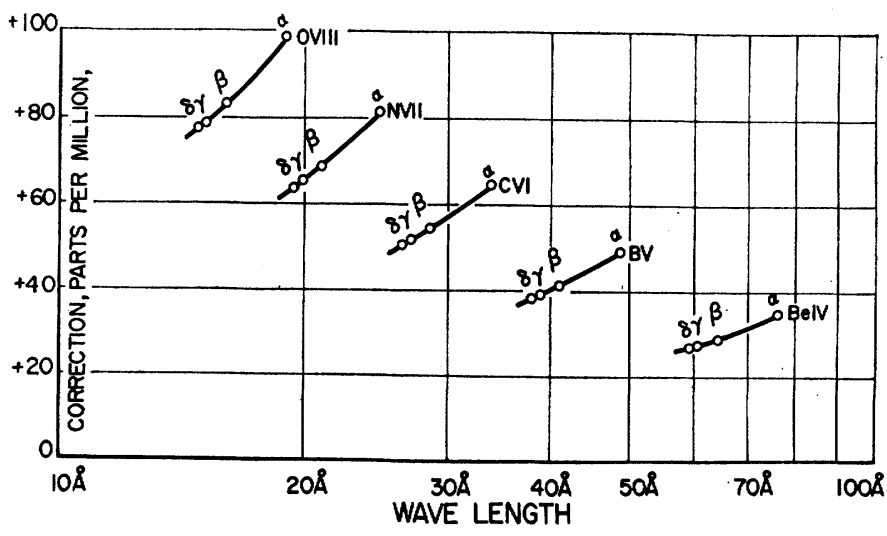

Fig. 2-Graph showing Lamb shift corrections in parts per million which must be made to calculated Lyman series spark-line wavelengths of one-electron atoms.

possible to give more than a rough estimate of what change the Lamb shift produces in Tyrén's published results. My colleague, Cohen, has made such an estimate based on the assumption that each X-ray line was associated in every case with that calibration line most nearly adjacent to it in wavelength and he thus has arrived at a corrected weighted mean value of $\Lambda$ for Tyrén's dissertation of

$\Lambda=1.002026 \pm 0.000016$ (Tyrén corrected for Lamb shift).

Taking a similarly corrected value for Bäcklin's work of 1.00203 and taking Bearden's 1935 work at the average value of 1.00208 , an equally weighted average of these three yields

$$
\Lambda=1.002045 \text { (tentative estimated grand mean). }
$$

I should like to emphasize that these are only tentative estimates and that the uncertainty as to how to correct 
TABLE III

Tyrén's Measurement of $\Lambda$ and Its Correction for the Lamb Shift

Tyrén's calibration wavelengths, Lyman series lines of $\mathrm{O}^{\mathrm{VIII}}, \mathrm{N}^{\mathrm{VII}}, \mathrm{C}^{\mathrm{VI}}, \mathrm{B}^{\mathrm{V}}, \mathrm{Be}^{\mathrm{IV}}$, were calculated using a formula in conformity with the Sommerfield-Dirac theory of the one-electron atom. The Lamb shift corrections, $\Delta E / E$, to this formula in ppm are:

\begin{tabular}{|c|c|c|c|c|c|c|}
\hline$n$ & & $\mathrm{O}^{\text {VIII }}$ & $\mathrm{N}^{\mathrm{VII}}$ & $\mathrm{C}^{\mathrm{VI}}$ & $\mathrm{B}^{\mathrm{v}}$ & $B e^{I V}$ \\
\hline $\begin{array}{l}1 \\
2 \\
3 \\
4\end{array}$ & $\begin{array}{l}\text { Lyman alpha } \\
\text { Lyman beta } \\
\text { Lyman gamma } \\
\text { Lyman delta }\end{array}$ & $\begin{array}{l}98.5 \mathrm{ppm} \\
84.0 \\
79.0 \\
77.5\end{array}$ & $\begin{array}{l}81.5 \mathrm{ppm} \\
69.0 \\
65.5 \\
63.5\end{array}$ & $\begin{array}{l}64.5 \mathrm{ppm} \\
54.7 \\
52.0 \\
50.4\end{array}$ & $\begin{array}{l}49.0 \mathrm{ppm} \\
41.0 \\
39.1 \\
38.3\end{array}$ & $\begin{array}{l}34.5 \mathrm{ppm} \\
29.0 \\
27.5 \\
26.8\end{array}$ \\
\hline
\end{tabular}

$$
\text { For np - ls transitions } \Delta E / E=\frac{8 n^{2}}{3 \pi\left(n^{2}-1\right)}\left(7.723-2 \ln Z-0.0439 / n^{3}\right)
$$

Tyrén's original uncorrected value was $\Lambda=1.00199$ (Dissertation, Uppsala, 1940)

After estimated correction (based on assumption that calibration lines were associated with X-ray lines closest to them in wavelength):

Tyrén corrected for Lamb shift $\quad \Lambda=1.002026 \pm 0.000016 \quad$ (Cohen, 1956)

Tentative Grand Mean of Bearden, 1.00208, Tyrén (corrected) 1.002026, and Bäcklin (corrected) 1.00203:

$$
\Lambda=1.002045 \quad \text { (Cohen, 1956) }
$$

Let $\Gamma_{\Lambda j}=\left(\Delta C_{i} / C_{i}\right) /(\Delta \Lambda / \Lambda)$ be the relative change in any atomic constant, $C_{i}$, per unit relative change in $\Lambda$. We estimate for our latest (1955)

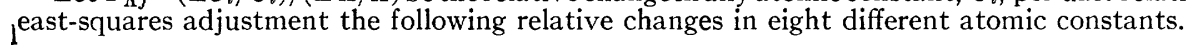

\begin{tabular}{cc|c|c|c|c|c|c|c}
\hline$C_{i}$ & $e$ & $m$ & $h$ & $\alpha$ & $\Lambda$ & $N$ & $F$ & $h c^{2} /(e \Lambda)$ \\
$\Gamma_{\Lambda i}$ & 0.686 & 1.108 & 1.285 & 0.087 & 1.000 & -1.035 & -0.348 & -0.401 \\
\hline
\end{tabular}

( $h c^{2} / e \Lambda$ is the voltage-wavelength conversion factor in $\mathrm{kv} \mathrm{X}$ units)

If the shift in our input value of $\Lambda$ turns out to be $25 \mathrm{ppm}$ (from 1.00202 to 1.002045 ), scarcely any of our tabulated constants, except $\Lambda$, will be shifted by a whole tabulated standard deviation.

Tyrén's results makes it impossible, at present, to do more than place rather widely separated upper and lower bounds on the magnitude of the upward shift (between $27 \mathrm{ppm}$ and $100 \mathrm{ppm}$ ).

It seems apparent, however, that the discrepancy between the American results on $\Lambda$, as obtained principally by Bearden [25] without recourse to calculated calibration lines and the lower Swedish values, is essentially to be explained in favor of the higher American values because of the need for the Lamb shift correction. At the University of Wisconsin, Prof. J. Mack has very courteously and generously placed at our disposal his 21-foot concave grating vacuum spectrometer, and my colleague and long-time friend and associate in research, Prof. H. A. Kirkpatrick, is now at work upon a redetermination of $\lambda_{s} / \lambda_{s}$ by the method of Tyrén.

A change in the input value of $\Lambda$, adopted for our least-squares adjustments, propagates its effect to greater or less extent into the least-squares adjusted values of every constant and conversion factor we have calculated in our output tables. For example, an increase of one part per million in $\Lambda$ is estimated to produce a change in each of the constants of the tabulation near the bottom of Table III, which is given in parts per million by the accompanying numeric in that table.

It is of considerable interest that I have just learned of some unpublished work of Bjorkman at Uppsala subsequent to Tyrén's by the same method, in which the value $\Lambda=1.00216 \pm 0.00004$ was obtained, a shift of 140 ppm from the Bragg edict of 1947 !

If the shift in $\Lambda$ turns out to be only $25 \mathrm{ppm}$ (from 1.00202 to 1.002045 ) scarcely any of the constants and conversion factors given in our tables, with the possible exception of $\Lambda$ itself, will be shifted by as much as one whole standard deviation as therein tabulated. A change of $140 \mathrm{ppm}$ would of course have much more serious consequences.

4. The Siegbahn-Avogadro Number, $N_{s}{ }^{\prime}=N \Lambda^{3}$. (By the $X$-Ray-Crystal-Density, XRCD, Method)

The philosophy of the X-ray-crystal-density (XRCD) determinations of Avogadro's number, $N$, reverses the order of reasoning followed by Siegbahn which we have just previously sketched. If the length of one edge of the unit cell in a cubic crystal is denoted by $d$, and the density of the crystal by $\rho$, then $d^{3} \rho$ is the mass of the unit cell. If there are $f$ molecules per unit cell, and if $M$ is the molecular weight, then $d^{3} \rho / f$ is the mass per molecule and Avogadro's number, $N$, the number of molecules in a gram mole, will be the ratio of these last two quantities, i.e., $N=M f /\left(\rho d^{3}\right) . M$ and $N$ must of course be expressed on the same scale of atomic weights. For crystals in general, the volume of a unit cell is given by $\phi d^{3}$ where $\phi$ is a geometric factor. ${ }^{6}$

The grating constant, $d$, is measured by measuring the Bragg angle for critical reflection of some X-ray spectral line of known wavelength in $\mathrm{X}$ units reflected by the atomic planes whose grating constant, $d$, is sought. If we use $d_{x}$ to indicate that the crystal spacing is in $\mathrm{X}$ units we have:

${ }^{6}$ For the calcite rhomb, $\phi$ is the volume of a rhombohedron whose three equal obtuse dihedral angles, $\alpha$, have the value, $105^{\circ} 3.5^{\prime}$ and the distances between whose three parallel pairs of faces is unity. For this case Bearden obtained $\phi=1.09594 \pm 0.00001$. 


$$
N=M f /\left[\rho \phi d_{x}^{3} \Lambda^{3}\right] .
$$

The quantity which the XRCD measurements really determine is, therefore

$$
N_{s}^{\prime}=N \Lambda^{3}=M f /\left[\rho \phi d_{x}^{3}\right],
$$

for which we shall coin the name, the SiegbahnAvogadro number, since it is the result of computing Avogadro's number from the measured properties of a crystal whose grating constant, $d$, has been measured on the Siegbahn nominal scale of $\mathrm{X}$ units.

We purposely avoid drawing any inference at this point as to the Avogadro number, $N$, by combining this directly measured value of $N \Lambda^{3}$ with the directly measured value of $\Lambda$. This is in accord with an important general principle to which we have consistently adhered throughout, namely, that the items of observational data entering into the least-squares adjustment must be kept observationally independent in order to simplify the assignment of their correct weights. I shall return to and emphasize this important point later.

Birge [26] has reviewed the determinations of $N$ based on five different crystals: calcite, rock salt, diamond, lithium fluoride, and potassium chloride. (See Table IV.) From these data the weighted mean value

\section{TABLE IV}

Probable Errors in Parts per Million for Factors of $N_{s}{ }^{\prime}$ and the Resulting Values

\begin{tabular}{l|c|c|c|c|c|c}
\hline \multicolumn{1}{c|}{ Crystal } & $M$ & $\rho_{20}$ & $d_{20}{ }^{3}$ & $\phi(\beta)$ & $\begin{array}{r}\text { Resulting } \\
\text { Error in } \\
N_{s}^{\prime}\end{array}$ & $\begin{array}{c}N_{s}^{\prime} \\
\left(10^{23} \mathrm{~mol}^{-1}, \text { chem }\right)\end{array}$ \\
\hline Calcite & 50 & 37 & 16.5 & 13 & 65 & $6.05989 \pm 0.00039$ \\
NaCl & 51 & 37 & 60 & & 87 & $6.06077 \pm 0.00052$ \\
Diamond & 20 & 34 & 89 & & 97 & $6.06018 \pm 0.00059$ \\
$\mathrm{LiF}$ & 32 & 45 & 120 & & 132 & $6.05994 \pm 0.00080$ \\
$\mathrm{KCl}$ & 40 & 37 & 68 & 86 & $6.05999 \pm 0.00052$ \\
\hline
\end{tabular}

of $N_{s}{ }^{\prime}$, the Siegbahn-Avogadro number on the chemical scaie of atomic weights, has the values shown on the table, first on the chemical and finally on the physical scale.

$$
\begin{aligned}
N_{s}{ }^{\prime}= & N \Lambda^{3}=(6.06014 \pm 0.00023) \times 10^{23}(\mathrm{~g} \mathrm{~mol})^{-1}(\text { chemical }) \\
& =(6.06179 \pm 0.00023) \times 10^{23}(\mathrm{~g} \mathrm{~mol})^{-1}(\text { physical }) .(12)
\end{aligned}
$$

Smakula and Sils, at the Massachusetts Institute of Technology, and Brogren, at Göteborg, Sweden, have made further determinations of densities and grating constants of crystals which add their support to the data presented here.

\section{5. $\gamma_{p}$, The Gyromagnetic Ratio of the Proton}

It is well known that atomic nuclei exhibit the properties of angular momentum ("spin") and magnetic dipole moment, with the magnetic dipole axis along the axis of spin. When a proton is placed in a magnetic field, one may think, to use a classical picture, of a torque exerted on the proton dipoles which tends to align them with the field. In view of their spin, however, they respond to the externally applied torque by precessing around an axis parallel to the external field with a frequency of precession which is proportional to the applied external magnetic field. The experiments at Harvard [27] and at Stanford [28] have shown that this "precession frequency" of the spinning protons can be measured very simply by placing a sample of water, sealed in a glass bulb, in a constant homogeneous magnetic field. A coil of wire, supplied with radio-frequency current and with its axis perpendicular to the magnetic field, surrounds this sample. When the magnetic field of the applied radio-frequency resonates with the precession frequency of the protons, an exchange of energy occurs, and the resulting perturbation of the precessing protons causes them to induce a detectable "signal" either in the applied radio-frequency coil itself (thereby changing its apparent impedance), or in a second detecting coil at right angles to the first.

The Harvard method utilizes a single coil, the Stanford method two coils. The ratio, $\gamma_{p}$, between the proton precession frequency, $\omega_{p}$, and the magnetic field intensity, $B$, is an extremely important constant or conversion factor because the method of proton resonance has proven itself to be by far the most convenient and accurate absolute method of measuring magnetic field intensities when these are in the range above a few hundred gauss.

In a hydrogen molecule the external applied magnetic field, $B_{a}$, differs slightly from the magnetic field, $B$, at the proton because of the diamagnetic effect of the electrons. The measured precession frequency, $\omega_{p}$, the apparent gyromagnetic ratio, $\gamma_{p}{ }^{\prime}$ (before diamagnetic correction), and the externally applied field $B_{a}$ are related by

$$
\omega_{p}=\gamma_{p}{ }^{\prime} B_{a}
$$

Ramsey [30] has computed the correction to the field for diamagnetism for the $H$ molecule. He obtained (see Fig. 3)

$$
-2.7 \times 10^{-5} B
$$

The gyromagnetic ratio of the proton has been measured at the U.S. National Bureau of Standards by Thomas, Driscoll, and Hipple [29], with a precision of about $22 \mathrm{ppm}$. In this experiment the field distribution between the pole pieces of an electromagnet first was explored carefully by means of a small proton resonance detector.

The field was then measured by weighing the force exerted on a rectangular current-carrying coil of exactly measured dimensions whose lower end was suspended between the pole pieces. The result was 


$$
r_{p}^{\prime}=\omega_{p} / B_{a}
$$

Field distribution between pole pieces of

an electromagnet was first carefully ex-

plored with a proton resonance head. The

field $B_{a}$ was then measured in absolute units

by weighing the force exerted on a rectangular

current-carrying coil of accurately measured

dimensions with its lower end suspended in the

field. H. A. Thomas, R. I. Driscoll, and

J. A. Hipple, National Bureau of Standards,

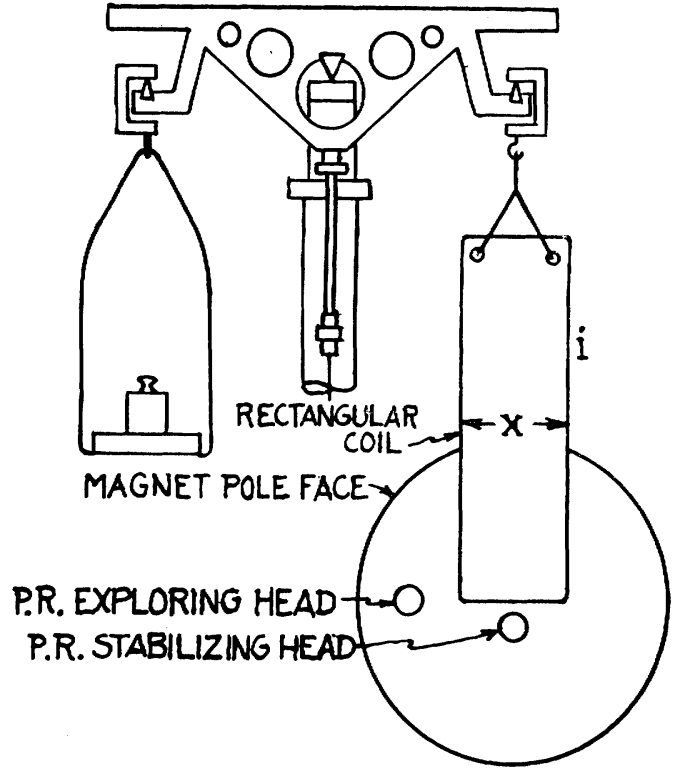

Journal of Research 쓰, 569 (1950); Phys.

Rev. 78, 787 (1950) obtained at United States National Bureau of Standards:

$$
r_{p}^{\prime}=\omega_{p} / B_{a}=\mu^{\prime} \mathrm{Ne} /\left(M_{p} c\right)=(2.67523 \pm 0.00006) \times 10^{4} \text { radians } \text { sec }^{-1} \text { gauss }^{-1}
$$

Fig. 3-Measurement of $\gamma_{p}{ }^{\prime}$, gyromagnetic ratio of the proton (before diamagnetic correction).

$$
\begin{aligned}
\gamma_{p}^{\prime} & =\omega_{p} / B=(2.67523 \pm 0.00006) \\
& \times 10^{4}{\text { radians } \sec ^{-1} \text { gauss }^{-1} .^{7}}
\end{aligned}
$$

6. $\omega_{c} / \omega_{p}$, Ratio of the Cyclotron Frequency of the Proton to its Magnetic Resonance Frequency (The Proton Magnetic Moment in Nuclear Magnetons)

The term "cyclotron frequency of the proton" is related to the well-known underlying principle of the simple magnetic resonance particle accelerator of $\mathrm{E}$. O. Lawrence, the constant field, constant frequency cyclotron. A particle with charge-to-mass ratio, $e / m$, in a magnetic field of constant intensity, $B$, executes circular orbits with an angular frequency of rotation which, at nonrelativistic speeds, is independent of the radius of the orbit. If the charge, $e$, is expressed in esu, this frequency is given by

$$
\omega=B e /(m c) \text {. }
$$

When the charged particle is a proton, the angular resonance frequency so obtained is the "proton cyclotron frequency," $\omega_{c}$, and if we measure the magnetic

${ }^{7}$ A slightly different result, $\gamma_{p}{ }^{\prime}=(2.67549 \pm 0.00016) \times 10^{4}$ radians $/ \mathrm{sec}^{-1}$ gauss $^{-1}$, higher by about one part in $10^{4}$, has recently been obtained by F. Kirchner and W. Wilhelmy of Cologne, Ger., using an iron-free solenoid. This was reported at the Avogadro Memorial Congress, Turin, Italy (Nuovo Cim., suppl., 1957). These authors suggested that the discrepancy might be attributable to difference in electrical standards. Huntoon and $\mathrm{McNish}$, of the U. S. National Bureau of Standards, suggest that the difference might be from use of an erroneous value of the acceleration of gravity which enters differently in the two types of determination of $\gamma^{\prime}$. field intensity, $B$, by the method just previously described in terms of the proton resonance frequency, $\omega_{p}$, we obtain an important measure of the charge-to-mass ratio $e / m_{p}$ of the proton, a datum of great interest for our knowledge of the atomic constants. Combining our equations for $\omega_{c}$ and $\omega_{p}[(14)$ and (15) $]$ one sees that the ratio, $\omega_{c} / \omega_{p}$, the cyclotron frequency of the proton to the proton magnetic resonance frequency is a universal constant

$$
\omega_{c} / \omega_{p}=\left(e / m_{p} c\right) \gamma_{p}{ }^{\prime-1}
$$

whose reciprocal is, in fact, the apparent magnetic moment of the proton, $\mu^{\prime}$ (uncorrected for diamagnetism),${ }^{8}$ expressed in nuclear magnetons, $\mu_{n}=e h / 4 \pi m_{p} c$ where $e$ is expressed in absolute esu and $m_{p}$ is expressed in grams.

To determine $\omega_{c}$, Sommer, Thomas, and Hipple [32], at the U.S. National Bureau of Standards, built a miniature cyclotron which they called the "omegatron" because it determined the angular frequency, $\omega_{c}$. In the omegatron the maximum orbit radius was only $1 \mathrm{~cm}$. The high vacuum $\left(10^{-7} \mathrm{~mm} \mathrm{Hg}\right)$ in this smail apparatus,

8 If one desires to know the absolute magnetic moment of the proton, $\mu$, the above-mentioned small correction must be made for the fact that the magnetic field intensity at the proton is not exactly the same as the externally measured magnetic field, because of the diamagnetic effect of the proton-containing sample. The diamagnetic effect for hydrogen gas, as we have said, has been computed by Ramsey [30] and the small additional shifts, of the order of a part in $10^{6}$, when water or mineral oil are used have been measured by Thomas [31]. 

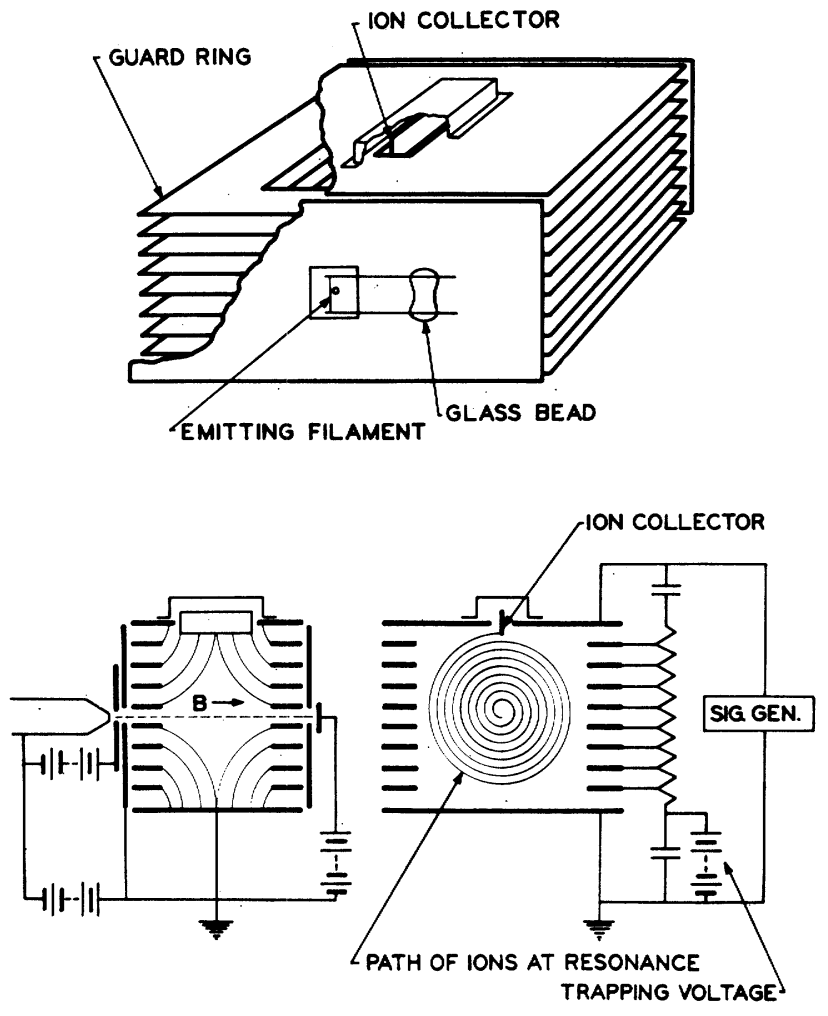

END VIEW

SIDE VIEW

Fig. 4-The "omegatron" of Sommer, Thomas, and Hipple.

shown schematically in Fig. 4, contained residual hydrogen, and ions (protons) were formed along an axis parallel to the magnetic field in the center of the device by impact from an axial beam of electrons. At right angles to the magnetic field a spatially uniform radio-frequency electric field (of variable frequency and of order $7 \mathrm{mc}$ ) accelerated ions of a selected charge-to-mass ratio at their cyclotron resonance frequency in spiral orbits until they attained a radius of $1 \mathrm{~cm}$, at which point they struck a collector. The resulting current was measured with an electrometer tube amplifier. An ion having a particular charge-to-mass ratio could attain a radius sufficient to reach the collector only when the frequency was tuned for ions of this type. The frequency width of the resonance peak so established depends on the number of orbital revolutions which the proton makes while it is being accelerated from zero radius out to the radius of the collector. It was possible to make this of the order of several thousand so that the resolution was extremely sharp. The magnet furnishing the field was the same one used in determining $\gamma_{p}$, and the cyclotron frequency of the proton was thus measured in relation to the nuclear resonance frequency of the proton. For this purpose the two-resonance devices (cyclotron and nuclear) could be quickly interchanged while a third nuclear resonance probe, through a servo-system employing the probe signal, held the field constant.

My time does not permit me to describe the ingenious way in which these experimenters corrected for the small source of error coming from the dc electric field required to insure axial stability to the proton orbits.
I must refer those interested in this correction to a footnote. ${ }^{9}$

Bloch and Jeffries [33], [34] have also measured the cyclotron frequency of the proton by a method differing from that of the omegatron, in that the protons were injected at high speed (a kinetic energy of 20,000 electron volts) and were decelerated by cyclotron action. They, therefore, called their device an "inverse cyclotron." (See Fig. 5.) It differed from the "omegatron" also in that "dees" were provided so that the region in which the protons encountered the high-frequency decelerating field constituted a very small fraction of a revolution. In consequence, it was possible to operate the device in "higher orders," that is to say at frequencies which were odd multiples of the frequency of revolution. Up to 11 half-cycles of the high frequency could occur during a half-revolution of the particles, and much additional resolution was thus gained.

The respective results of the "inverse cyclotron" and the "omegatron,"

$$
\begin{aligned}
& \mu^{\prime}=2.792365 \pm 0.000100(36 \mathrm{ppm}) \text { (inverse cyclotron) } \\
& \mu^{\prime}=2.792685 \pm 0.000030(11 \mathrm{ppm}) \text { (omegatron) }
\end{aligned}
$$

differ by only $115 \mathrm{ppm}$, but the difference is uncomfortably large relative to the standard deviations assigned to each experimental result. The difference is in fact three times its root-mean-square expectation value. Work has since been published by Collington, Dellis, Sanders, and Turberfield [35] who repeated the inverse cyclotron experiment at the Clarendon Laboratory, Oxford, Eng. They did not use conventional cyclotron "dees." Instead they had a central electrode with straight parallel sides to which the radio frequency was applied while two grounded segmental electrodes on either side completed the cylindrical box. With this arrangement the protons were decelerated to such a radius that they eventually attained orbital stability without further gain or loss of energy.

${ }^{9}$ A small source of systematic error was carefully studied. This came from the presence of a dc electric field which had to be provided to stabilize the proton orbits against axial drift. There must clearly be, in addition to the component of this dc field in the axial direction, a component in the radial direction, and this latter must exert forces and (unlike the magnetic field) do work on the spiralling protons. The force from this dc field is much smaller than the magnetic force but it depends on the radius, $\rho$, in a way not very dissimilar from the latter. At the resonant frequency, $\omega_{r}=B\left(e / m_{p} c\right)$, the normal magnetic force on the proton is $F_{\text {mag }}=\left(B e \omega_{r} / c\right) \rho$, while we may, to first approximation, express the radial component of the dc stabilizing field force as $F_{e 1}=-k \rho$. Thus the frequency, $\omega_{r}$, would be slightly shifted by this additional force. This effect was carefully studied by varying the magnitude of the electric stabilizing field over a considerable range and plotting a curve showing the slight variation of the apparent proton cyclotron frequency as a function of the dc stabilizing field. This curve turned out to be linear as expected and could be extrapolated to zero electric field so as to correct for the latter. However, these experimenters found a still better way to make this correction. This depends on the fact that, whereas the resonance frequency, $\omega_{r}$, is inversely proportional to the mass of the accelerated particle, the shift in frequency, $\Delta \omega$, due to the radial electric field, is independent of this mass. By determining in each case the resonant frequency for two different masses, e.g., $\mathrm{H}^{+}$and $\mathrm{H}_{2}+, \mathrm{H}^{+}$and $\mathrm{D}_{2}{ }^{+}$, and $\mathrm{H}^{+}$and $\mathrm{H}_{2} \mathrm{O}^{+}$, it was possible to evaluate the correction. After making this correction, the average deviation from the mean was one part in 40,000, a result which gives considerable confidence in the reliability of the correction. 


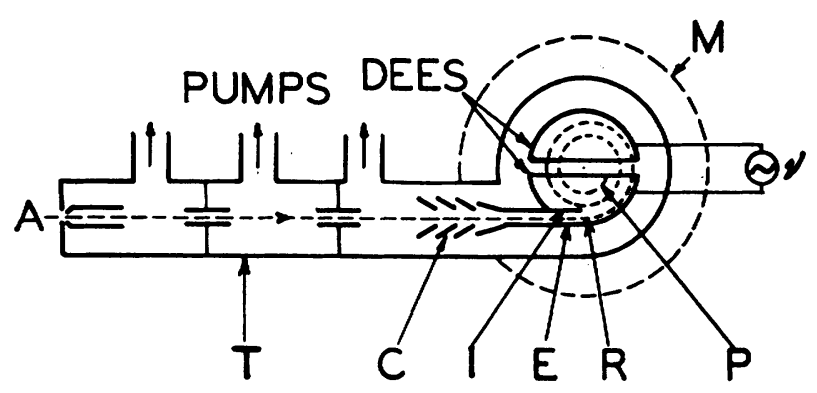

Bloch \& Jeffries' Original Form

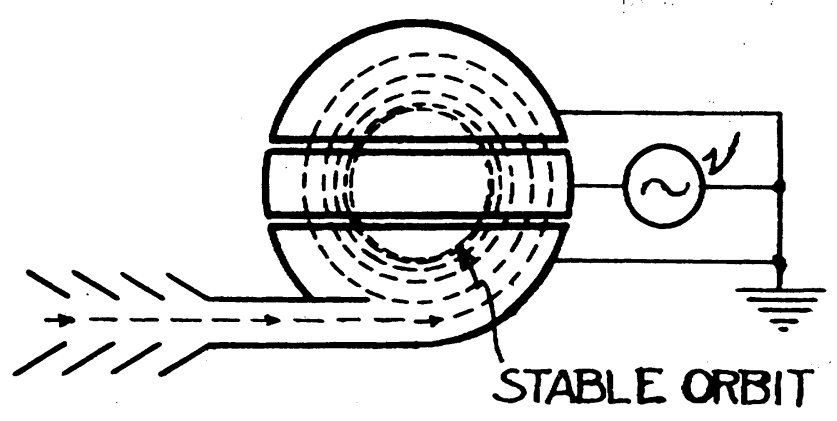

Collington, Dellis, Sanders \& Turberfield's Modification

Comparison of Results:-

B. \& J., Inverse Cyclotron

$$
\begin{aligned}
& \mu^{\prime}=2.792365 \pm 0.000100 \text { nuclear magnetons } \\
& \mu^{\prime}=2.792685 \pm 0.000030 " ~ " \\
& \mu^{\prime}=2.792730 \pm 0.000040 \\
& \mu^{\prime}=2.79267 \pm 0.00010 \quad "
\end{aligned}
$$$$
\text { S. T. \& } \mathrm{H}_{\bullet} \text {, "Omegatron" }
$$$$
\text { C. D. S. \& T., Stable Orbit }
$$$$
\text { Inverse Cyclotron }
$$

B. \& J. Inverse Cyclotron with

K. Trigger's Correction

Fig. 5-Two forms of the "inverse cyclotron," that of Bloch and Jeffries and that of Collington, Dellis, Sanders, and Turberfield.

E. S. Dayhoff, S. Triebwasser and Willis Lamb, Jr. Phys. Rov. 89, 98, 106 (1953) Important because of relationship to Sommerfeld fine structure constant, $a$. $\Delta E_{D}=(1 / 16) a^{2} R_{\infty} c\left[1+\frac{5}{8} a^{2}+\frac{a}{\pi}\left(1-5.946 \frac{a}{\pi}\right)\right] M_{d} / D=10971.59 \pm 0.10 \mathrm{Mc} / \mathrm{sec}$ (S.D. ) $\Delta E_{D}$ is the energy separation between the $2{ }^{2} \mathrm{P}_{3 / 2}$, and $2{ }^{2} \mathrm{P}_{1 / 2}$ states in deuterium.

Obtained by combining the measured LambRetherford shift, $s_{D}$, corresponding to $2^{2} \mathrm{~S}_{1 / 2}-2^{2} \mathrm{P}_{1 / 2}$ with the separation $\Delta E_{D}-S_{D}$ corresponding to $2{ }^{2} P_{3 / 2}-2^{2} S_{1 / 2}$. The $2^{2} \mathrm{~s}_{1 / 2}$ state is metastable, 30 microseconds half Iife. $2 P_{I / 2}$ and $2 P_{3 / 2}$ states are much shorter.

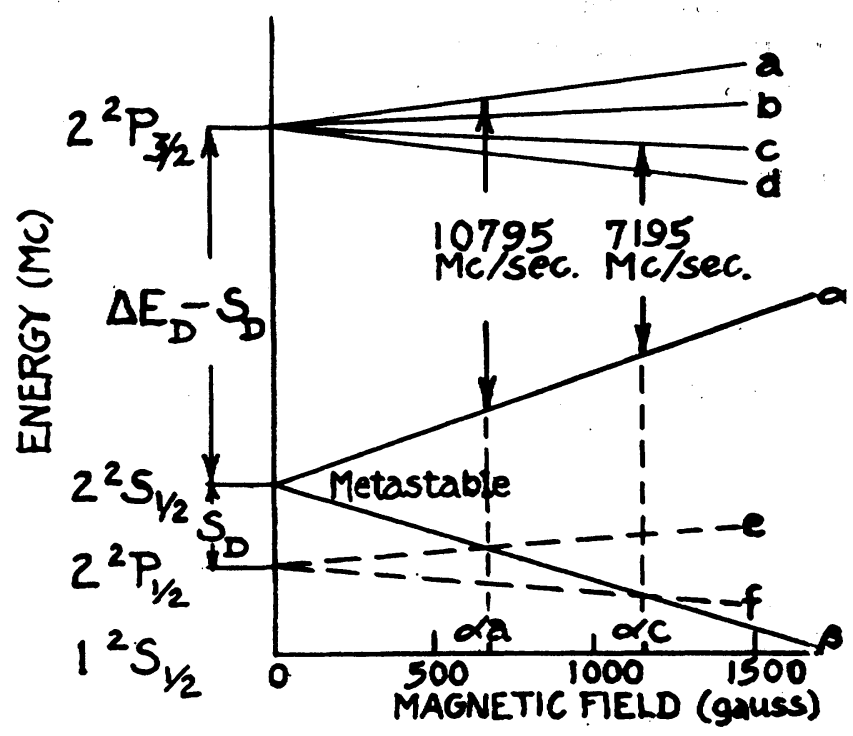

Fig. 6-Measurement of the fine structure separation in deuterium, $\Delta E_{D}$, in frequency units by Triebwasser, Dayhoff, and Lamb. 
The value of $\mu^{\prime}$ (uncorrected for diamagnetism) obtained by Collington, Dellis, Sanders, and Turberfield is $\mu^{\prime}=2.792730 \pm 0.000040(14 \mathrm{ppm})$ (inverse cyclotron with stable orbit),

which is in good agreement with the omegatron measurement.

More recently still, Trigger [36] has shown that theoretical calculations of the Bloch and Jeffries inverse cyclotron orbits yield a set of three coupled nonlinear equations which predict a frequency shift dependent upon the decelerating radio-frequency voltage. After these corrections Trigger finds that the inverse cyclotron results of Bloch and Jeffries are in good agreement with those of the omegatron, or of Collington, Dellis, Sanders, and Turberfield. Trigger's corrected value is (see Fig. 6)

$$
\mu^{\prime}=2.79267 \pm 0.00010 \text { nuclear magnetons. }
$$

\section{7. $\Delta E_{D}$ Fine Structure Separation in Deuterium in Fre- quency Units}

The energy separation, $\Delta E_{D}$, of the $2^{2} P_{3 / 2}$ and $2^{2} P_{1 / 2}$ states of deuterium has been measured in frequency units with the astonishing accuracy of $\pm 9 \mathrm{ppm}$ by Triebwasser, Dayhoff, and Lamb, at Columbia University [37]. This marked the culmination of a remarkable series of researches on the fine structure of the hydrogen and deuterium atoms by Lamb, with his coworkers [38]-[41]. It was through this series of researches that the existence of the "Lamb-Retherford shift," the energy difference between the $2^{2} S_{1 / 2}$ and $2^{2} P_{1 / 2}$ states, was established, and its value accurately measured. From the point of view of fundamental physics, the importance of the Lamb-Retherford shift greatly overshadows the precision measurements of the fine structure separation in deuterium. This latter is, however, of prime importance as a contribution to our knowledge of the atomic constants because of its relation to the Sommerfeld fine structure constant, $\alpha$, and it is, therefore, the only result by these workers which we shall discuss here.

Lamb and his coworkers actually obtained the separation, $2^{2} P_{3 / 2}-2^{2} P_{1 / 2}$, in deuterium by combining the results of two independent measurements. These were 1) the $2^{2} P_{3 / 2}-2^{2} S_{1 / 2}$, and 2) the separation $S_{D}=2^{2} S_{1 / 2}$ $-2^{2} P_{1 / 2}$, the famous "Lamb shift." Here we shall describe only the $2^{2} P_{3 / 2}-2^{2} S_{1 / 2}$ measurement, since the Lamb shift was accomplished by similar methods. It is important to note that the $2^{2} S_{1 / 2}$ state is metastable with a lifetime of order 13 or $14 \mu \mathrm{sec}$.

Fig. 7 is a cross section through the apparatus used by Lamb and his associates. The deuterium molecules pass through a tungsten tube heated to about $2500^{\circ} \mathrm{K}$ by electrical conduction and are dissociated into monatomic deuterium. After passing through a slit they enter a box in an accurately controlled and measured mag- netic field whose intensity may be varied from nearly zero to 4000 gauss. In this box the monatomic beam is bombarded with electrons of energy $10.8 \mathrm{v}$ so that excitation of the deuterium to its metastable $2^{2} S_{1 / 2}$ state occurs. ${ }^{10}$ Their lifetime is sufficient to permit a beam of deuterium atoms to persist in this excited state over a distance of 7 or $8 \mathrm{~cm}$ or more. When the metastable atoms strike a metal target, $J$, their energy of excitation is expended to eject electrons from it and these electrons are collected at $K$ and measured with an "electrometer tube" (dc amplifier) as a means of detecting the metastable beam. Between the exciting bombarder and the detector, however, the beam of atoms is subjected to a radio-frequency electric field and, if this frequency is exactly right, the metastable atoms undergo transitions to the non-metastable excited states $2^{2} P_{1 / 2}$ and $2^{2} P_{3 / 2}$ from which they decay with great rapidity ${ }^{11}$ to the ground state. In this state, no excitation energy being available, they are not detected. A decrease in the response at the detector is, therefore, the index of a resonance between the applied radio-frequency field and an atomic transition.

The purpose of the magnetic field, as originally planned, was to split the $2^{2} S_{1 / 2}$ and $2^{2} P_{1 / 2}$ states by the Zeeman effect in order to insure longer life to the $2^{2} S_{1 / 2}$ state. This was before the existence of the natural (LambRetherford) splitting had been established or realized. The magnetic field was also deemed useful to keep charged particles away from the detector. The third, and actually most important function of the magnetic field, however, turned out to be that of tuning the critical frequencies of the metastable atoms (through Zeeman effect) to the applied radio-frequency field, thus permitting the use of a rigorously constant, rather than a variable, applied radio frequency which, for technical reasons, is preferable in a high precision measurement of this sort.

The measurements of Triebwasser, Dayhoff, and Lamb [37] yielded the value

$$
\Delta E_{D}=10971.59 \pm 0.10 \mathrm{mc}
$$

for the fine structure separation in frequency units between the $2^{2} P_{3 / 2}$ and $2^{2} P_{1 / 2}$ states of deuterium. This, however, cannot be directly equated to the familiar expression, $\left(\alpha^{2} / 16\right) R_{\infty} c$, for the fine structure splitting, because this expression is not sufficiently accurate. Three corrections are required. In the first place $R_{\infty}$, the Rydberg constant for an infinitely heavy nucleus, must be replaced by the Rydberg constant for deuterium. This introduces the factor, $M_{D} / D$, the ratio of the mass of the deuteron to the mass of the neutral deuterium

${ }^{10}$ Even at this low bombarding energy the transverse recoil of the atoms is sufficient to introduce an angular spread in the beam of the order of three degrees. Lamb, therefore, has questioned the propriety of describing it as a "beam" in comparison with the beams of the better known technique developed by Rabi, Millman, Kusch, and Zacharias [61].

11 The atoms are estimated to move only a few microns in this decay time. 


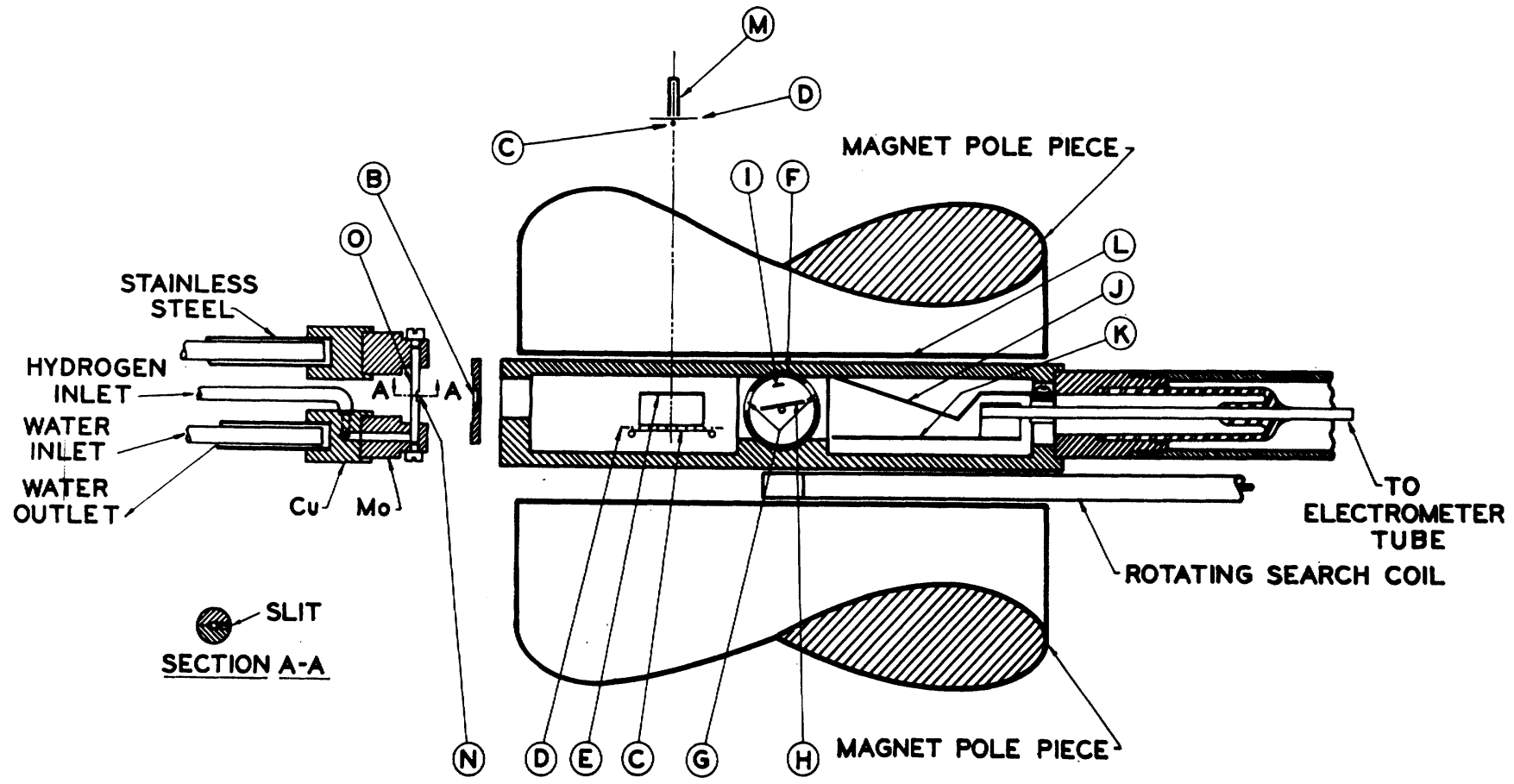

Fig. 7-Apparatus of Lamb.

The measured quantities are:--

$\lambda_{s}$ the wavelength setting of

the monochromator in kx-units (Siegbahn scale)

$\mathrm{V}_{\mathrm{A}}=\mathrm{V}_{\mathrm{H}}+\mathrm{V}_{\mathrm{W}}$.

$\mathrm{V}_{\mathrm{H}}=$ cathode-to-target voltage

$\mathrm{V}_{\mathrm{W}}=$ cathode work function
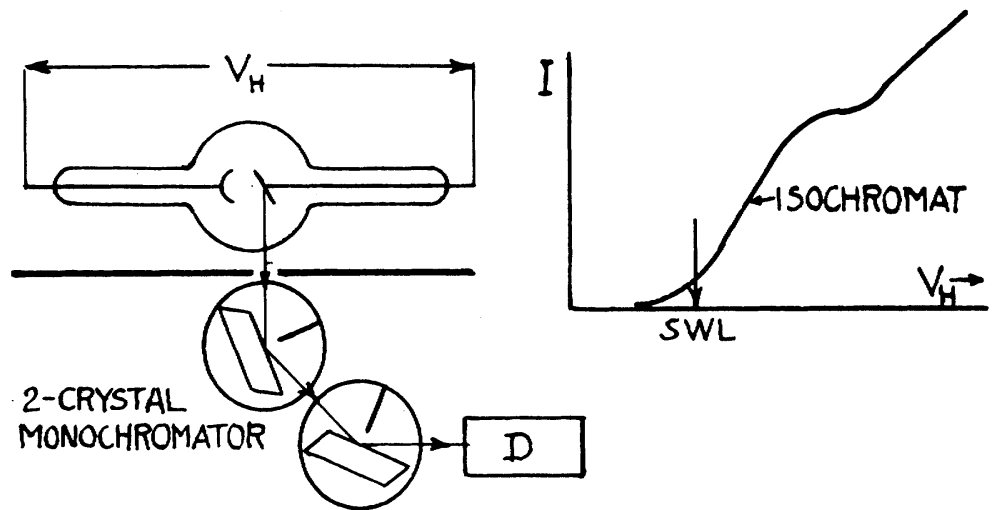

The voltage, $V_{H}$, is varied in small steps and the curve of $x$-ray intensity in the fixed wavelength domain defined by the monochromator, plotted as function of $\mathrm{V}_{\mathrm{H}}$, is called an "isochromat." From the threshold point on this isochromat the voltage $V_{A}$ at which the maximum frequency, $\nu_{m}$, or minimum wavelength, $\lambda_{g}$, of the continuous spectrum coincides with the peak of the "window" of the monochromator can be inferred. Then $(\mathrm{e} / \mathrm{c}) \mathrm{V}_{\mathrm{A}}=\mathrm{h} \nu_{\mathrm{m}}=\left(\mathrm{hc} / \lambda_{\mathrm{g}}\right) 10^{8}=\left(\mathrm{hc} / \lambda_{\mathrm{s}}\right) \Lambda^{-1} 10^{8}$ when $\Lambda=\lambda_{\mathrm{g}} / \lambda_{\mathrm{s}}, \lambda_{\mathrm{g}}$ in angstroms

Thus the quantity determined by this experiment is: -$\lambda_{s}$ in $k$ x-units

$$
\mathrm{V}_{\mathrm{A}} \lambda_{\mathrm{S}}=(\mathrm{h} / \mathrm{e}) \mathrm{c}^{2} \Lambda^{-1} 10^{8} \text {. (voltage-wavelength conversion factor) }
$$

The D.C. voltage difference, $V_{H}$, between cathode and target is usually measured by means of a high resistance potential divider. The P.D. across an accurately calibrated fraction of the divider is measured by means of a standard cell and a potentiometer.

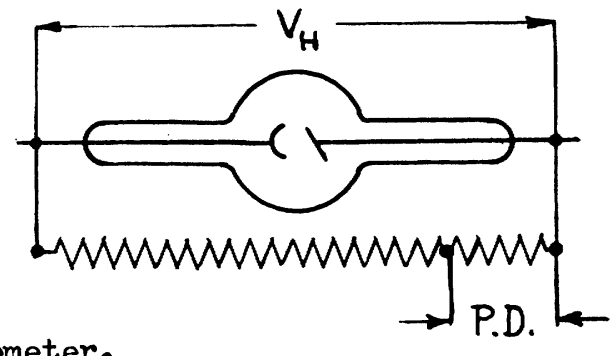

Fig. 8-To explain the determination of the voltage-wavelength conversion factor by means of the short wavelength limit of the continuous $\mathrm{X}$-ray spectrum. 
atom. Secondly, higher-order terms in the Dirac expression for the fine structure splitting must be included; this adds a correction term of relative amount (5/8) $\alpha^{2}$. These two corrections are both implicit in the Dirac theory, and their inclusion here is required by the increased accuracy of experimental techniques. The third correction represents a modification of the Dirac theory and is the result of the anomalous magnetic moment of the electron [42], [43]. It is properly identified with the Lamb-Retherford shifts in the energy of the $2^{2} P_{1 / 2}$ and $2^{2} P_{3 / 2}$ levels [44], [45]. The correct theoretical expression for the energy difference has been given by Lamb, accurate to terms of order $\alpha^{6} \mathrm{mc}^{2}$. This expression is ${ }^{12}$

$$
\begin{aligned}
\Delta E_{D}=(1 / 16) \alpha^{2} R_{\infty} c & {\left[1+(5 / 8) \alpha^{2}\right.} \\
+ & \left.\frac{\alpha}{\pi}\left(1-5.946 \frac{\alpha}{\pi}\right)\right] M_{d} / D .
\end{aligned}
$$

8. The Voltage-Wavelength Conversion Factor, $(h / e) c^{2} \Lambda^{-1}$ (Short Wavelength Limit of the Continuous X-Ray Spectrum)

X-ray SWL determinations consist in exciting an $\mathrm{X}$-ray tube with an extremely stable and very accurately measured dc voltage, $V_{H}$, and simultaneously determining with a spectrometer of highest possible resolution the minimum wavelength, $\lambda_{m}$, of the continuous $\mathrm{X}$-ray spectrum emitted by the tube. It has been shown [46]-[49], both by theoretical considerations and by internal experimental evidence that the quantum energy, $h \nu_{m}=h c / \lambda_{m}$, of the radiation at this limiting point corresponds ideally to the energy acquired by the thermally emitted cathode electrons falling through a potential difference, $V_{A}=V_{H}+V_{W}$, which is the sum of $V_{H}$, the measured voltage difference between cathode and target, and $V_{W}$, the work function of the thermally emitting cathode.

The voltage, $V_{H}$, is varied in small steps and the curve of X-ray intensity in the fixed wavelength domain defined by the monochromator, and plotted as a function of $V_{H}$, is called an "isochromat." From the threshold point on this isochromat one ascertains the voltage, $V_{A}$, at which the minimum wavelength component of the continuous spectrum coincides with the peak of the band-pass or "window" curve of the monochromator. For this point, conservation of energy requires the relation

$$
\begin{aligned}
(e / c) V_{A}= & h \nu_{m}=\left(h c / \lambda_{g}\right) 10^{8} \\
= & \left(h c / \lambda_{s}\right) \Lambda^{-1} 10^{8} ; \lambda_{o} \text { in angstroms } \\
& \lambda_{s} \text { in kilo X units. }
\end{aligned}
$$

Thus the quantity determined by this experiment is

$V_{A} \lambda_{s}=(h / e) c^{2} \Lambda^{-1} 10^{8}$ (voltage-wavelength conversion

factor in $\mathrm{kv} \mathrm{X}$ units).

The dc voltage, $V_{H}$, applied to the $\mathrm{X}$-ray tube terminals is usually measured by means of a high-resistance potential divider, a precisely calibrated fraction of the potential drop being measured with a potentiometer and standard cell.

This experiment has always tended to yield values of $h / e$ which were lower than the value required for consistency with the bulk of the data on the constants, i.e., in the direction implying more radiant quantum energy, $h \nu$, than the electron energy, $e V_{A}$, which produced it. Older, more obvious sources of such error gradually have been eliminated. Unfortunately the results of all of even the most recent and carefully performed experiments of this type are still somewhat incompatible (and in the same direction) with the remaining data of our otherwise quite consistent leastsquares adjustments. The discrepancy is now roughly of the order of a part in ten thousand. ${ }^{13}$

Table $\mathrm{V}$ shows the results of nine determinations of

\begin{tabular}{|c|c|c|c|c|c|c|}
\hline $\begin{array}{l}\text { Experi- } \\
\text { menters }\end{array}$ & $\begin{array}{l}\text { Nominal } \\
\text { Voltage }\end{array}$ & $\begin{array}{c}a \\
\text { (volt) }\end{array}$ & $\begin{array}{c}h c^{2} / e \Lambda \\
\left(\mathrm{kv} \mathrm{X}^{\mathrm{units}}\right)\end{array}$ & $\begin{array}{l}\text { Discrepancy } \\
\quad(\mathrm{ppm})\end{array}$ & $\begin{array}{c}\text { Discrepancy } \\
\text { (volt) }\end{array}$ & Remarks \\
\hline $\begin{array}{l}\mathrm{BJW}^{1} \\
\mathrm{BS}^{2} \\
\mathrm{BS}^{2} \\
\mathrm{BS}^{2} \\
\mathrm{BS}^{2} \\
\mathrm{BJW}^{1} \\
\mathrm{BS}^{2} \\
\mathrm{BS}^{2} \\
\mathrm{FHD}^{3}\end{array}$ & $\begin{array}{r}6,112 \\
8,050 \\
8,050 \\
8,050 \\
9,860 \\
10,168 \\
19,600 \\
19,600 \\
24,500\end{array}$ & $\begin{array}{l}0.27 \\
0.37 \\
0.37 \\
0.37 \\
0.47 \\
0.48 \\
0.96 \\
0.96 \\
6.0\end{array}$ & $\begin{array}{l}12,370.8 \\
12,371.9 \\
12,371.0 \\
12,370.1 \\
12,370.1 \\
12,371.2 \\
12,371.9 \\
12,370.1 \\
12,370.0\end{array}$ & $\begin{array}{l}-116 \\
-28 \\
-101 \\
-174 \\
-174 \\
-80 \\
-28 \\
-174 \\
-177\end{array}$ & $\begin{array}{l}-0.7 \pm 0.4 \\
-0.2 \pm 0.5 \\
-0.8 \pm 0.5 \\
-1.4 \pm 0.5 \\
-1.7 \pm 0.5 \\
-0.8 \pm 0.6 \\
-0.6 \pm 1.1 \\
-3.4 \pm 1.1 \\
-4.3 \pm 1.3\end{array}$ & $\begin{array}{l}\text { W target } \\
\text { Cu target } \\
3 \text { observations Mo, Ta, Au targets } \\
\text { W target } \\
2 \text { observations } \mathrm{Cu}, \mathrm{Ni} \text { targets } \\
\text { W target } \\
\text { W target; W fil. } \\
\text { W target; ox. cath. } \\
\text { W target; W fil." }\end{array}$ \\
\hline
\end{tabular}
the voltage-wavelength conversion factor measured at six different voltages ranging from 6112 to $24,500 \mathrm{v}$.

TABLE V

X-Ray Quantum Limit Determinations

1 J. A. Bearden, F. T. Johnson, and H. M. Watts.

2 J. A. Bearden, and G. Schwarz.

${ }^{3}$ G. F. Felt, J. N. Harris, and J. W. M. DuMond.

12 An error in the 1951 theoretical calculations of $\mu_{e} / \mu_{0}$ by Karplus and Kroll [43], which was not discovered until late in 1957, now requires that the numeric, -5.946 , in (21) (which is twice the coefficient of the fourth-order term in the expression for $\mu_{e} / \mu_{0}$ ), be changed to -0.656. This information came too late for inclusion in our last (1955) adjustment. See Part III, Sec. 20 and 23.

${ }^{13}$ Of course, if it should transpire that the wavelength conversion constant $\Lambda$ must be revised upward by 100 or even $140 \mathrm{ppm}$, this might completely eliminate the " $h / e$ discrepancy." The unpublished work of $B j o ̈$ orkman on $\Lambda$, referred to, indicated this as a possibility. 
A trend, which we believe is indicative of a systematic error more or less common to all the experiments listed, is perceptible if we plot the discrepancy in volts between the observed and computed threshold values as a function of the different experimental voltages at which they were determined. In this comparison we have based the computed values on our 1953 least-squares adjusted value of the voltage-wavelength conversion factor, namely

$$
h c^{2} /(e \Lambda)=12372.2 \mathrm{kv} \mathrm{X} \text { units. }
$$

The ordinates in Fig. 9 show the aforementioned discrepancies in volts plotted as a function of the voltage of each experiment. Where several values fall at the same point the number of these is indicated. As you can see there is a reasonably well-defined trend, the discrepancy in volts tending to diminish roughly linearly with voltage, although the precision is insufficient to guarantee a firm conclusion as to this linearity.

The criterion used in this experiment to locate the true threshold point on the "fillet" of the observed isochromat curve is to take it at the point of maximum "bending" (i.e., maximum second derivative) of the isochromat (see Fig. 10.) This method, first proposed by DuMond in 1937 and used in all the precision determinations which we are considering here, is based on

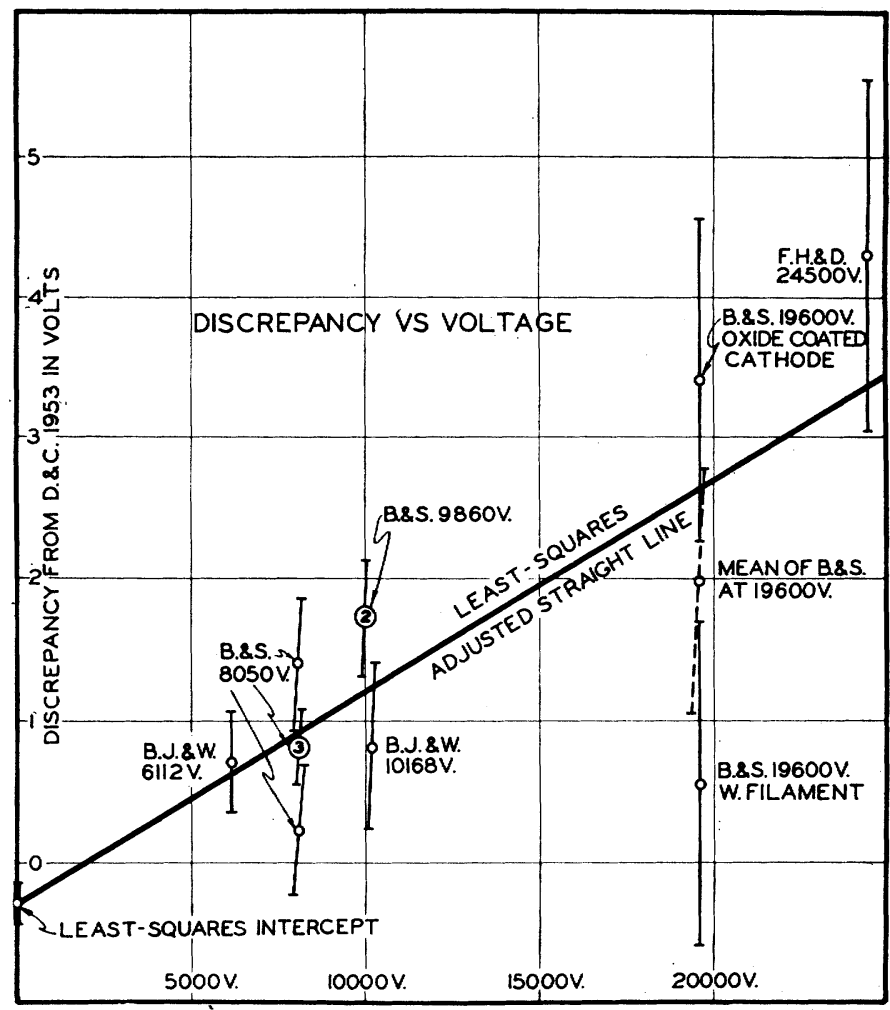

Fig. 9-Determinations of the quantum limit of the continuous $\mathrm{X}$-ray spectrum measured at six different applied voltages and plotted as a function of the applied voltage.

Basic Assumption:-- The true continuous spectrum profile for a thick target has

a second order discontinuity at the quantiom limit.

The observed iso-

chromat is the fold of the true isochromat into the "window curve," $\mathrm{g}$, of the spectrometer.

$$
F(z)=\int_{x=-\infty}^{x=z} g(x) f \cdot(z-x) d x
$$

Assume the true isochromat consists of a broken line consisting of straight segments with slopes:--

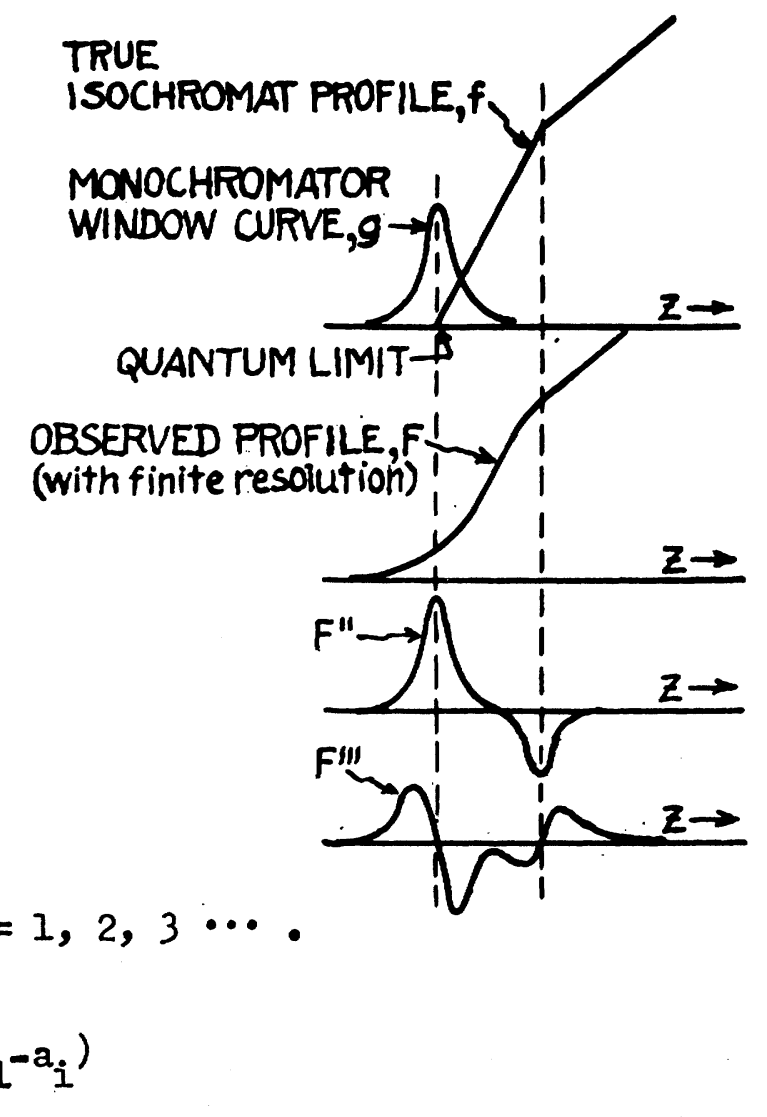

Then

$$
\begin{aligned}
& f^{\prime}(x)=a_{i} \text { for } x_{i-1}<x<x_{i} ; i=1, \\
& F^{\prime \prime}(z)=g(z) f^{\prime}(0)+\sum_{i=1}^{\infty} g\left(z-x_{i}\right)\left(a_{i+1}-a_{i}\right)
\end{aligned}
$$

Thus each break in slope in the f-curve generates in the Fn curve a replica of the g-curve whose intensity and sign is proportional to the break. The third derivative F'W will clearly have a "zero" at each break in slope.

Fig. 10-The criterion of maximum bending of the X-ray isochromat to locate the quantum limit of the continuous X-ray spectrum. 
the assumption that the true continuous spectrum profile for a thick target $\mathrm{X}$-ray tube has a simple secondorder discontinuity at the quantum limit. The observed isochromat, $F$, is the "fold" of the true spectral profile into the monochromator "window" curve, $g$.

$$
F(z)=\int_{x=-\infty}^{x=z} g(x) f(z-x) d x
$$

If we approximate the shape of the true spectral profile, $f$, in the neighborhood of the limit by a broken straight line with straight segments having finite slopes, $a_{j}$,

$$
f^{\prime}(x)=a_{i} \text { for } x_{i-1}<x<x_{i} ; \quad i=1,2,3,
$$

then differentiating our fold equation, (24), twice one obtains

$$
F^{\prime \prime}(z)=g(z) f^{\prime}(0)+\sum_{i=1}^{\infty} g\left(z-x_{i}\right)\left(a_{i+1}-a_{i}\right)
$$

from which we see that each break in slope in the true spectrum (including the second-order discontinuity at the quantum limit) generates in the $F^{\prime \prime}$ curve a replica of the $g$ (or window) curve whose intensity and sign is proportional to the magnitude of the break in slope. The third derivative, $F^{\prime \prime}$, of the isochromat will clearly have a "zero" at each break in slope.

Fig. 11 shows one of the isochromats obtained at the highest voltage, 24,500 , listed in the preceding tabulation. The point marked $A$ is the point of maximum bending; the point $B$, however, is the required position of the quantum limit for consistency with the 1953 least-squares adjustment.

Fig. 12 shows how the point of maximum bending ( $A$ in Fig. 11) was determined; by plotting the third derivative of the isochromat and locating its zero point. ${ }^{14}$ The point $B$, to the extreme right, is the required position for consistency with the 1953 adjustment.

The systematic trend indicated in the preceding tables and figures strongly suggesting a systematic error might be explicable if we postulate that the ideal structure of the quantum limit consists of a superposition of a first- and a second-order discontinuity as shown in the upper diagram of Fig. 13. Referring to the lower part of that figure, if $h$ is the height of the precipice, if $s=\tan \theta$ is the slope above it, and if $z_{h}=h / s$ is what we have called the "overshoot," it is easy to show that

$$
F^{\prime \prime}(z)=s g(z)+h g^{\prime}(z)=s\left[g(z)+z_{h} g^{\prime}(z)\right] .
$$

14 The third derivative was obtained by a numerical interpolation method using for each value of $F^{\prime \prime \prime}$ an array of points at equidistant abscissa spacings on the smoothed isochromat curve. The point at which $F^{\prime \prime}$ was to be evaluated was always at the center of this array. The method yields the third derivative of a high-order polynomial fitted to the points of the smoothed curve but is, of course, no more precise than the smoothing of the curve is reliable. The assigned uncertainty (indicated by the arrows in this and the previous figure), $F^{\prime \prime \prime}=0$ when the curve was smoothed in different trials and by different people

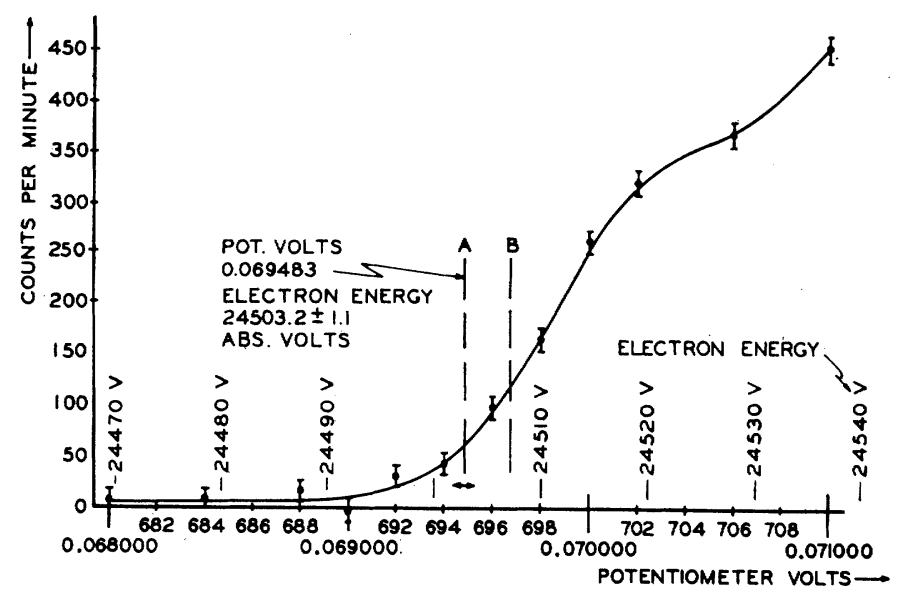

Fig. 11-Isochromat obtained at 24,500 v by Felt, Harris and DuMond. The point marked $A$ is the point of maximum bending; the point $B$ is the required position of the quantum limit for consistency with the 1953 adjustment.

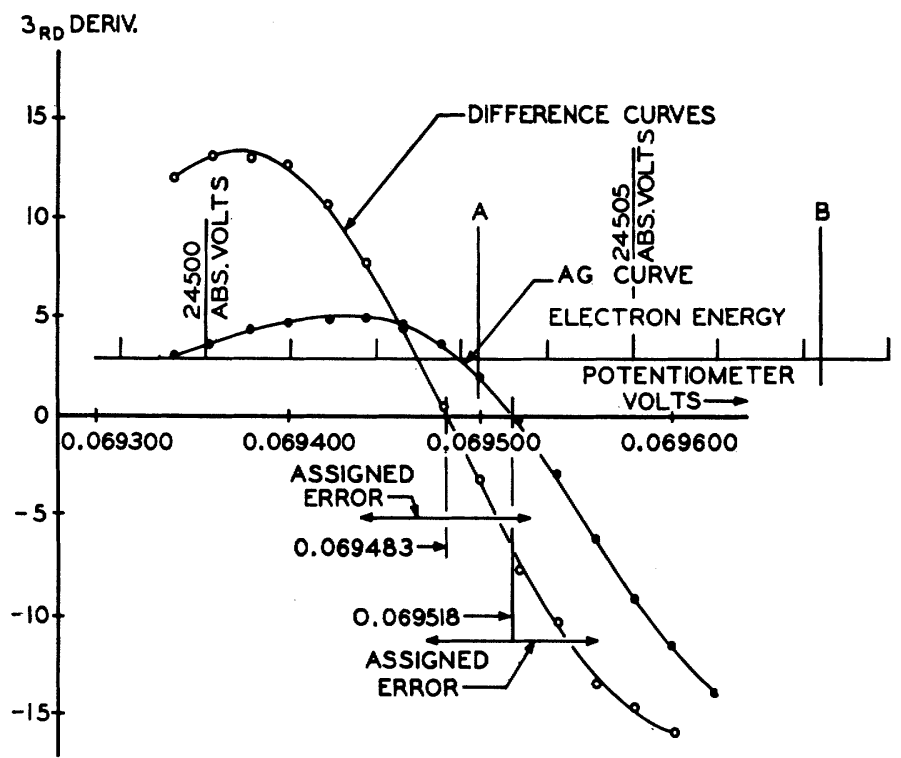

Fig. 12-The third derivative of the isochromat of Fig. 11. The mean of the zeropoint cross-overs of the curves located the position, $A$, in Fig. 11.

Clearly, whereas the second-order discontinuity generates a $g$ curve, the first-order discontinuity generates a curve which is the first derivative of the $g$ curve. (This follows because the first-order discontinuity or step function, considered by itself, is the limiting case of two equal breaks in slope of opposite sign which have approached indefinitely close to each other and have become indefinitely large.) The superposition of these two curves, the primitive $g$ curve and its first derivative, may indeed result in a peak in the $F^{\prime \prime}(z)$ which is considerably shifted from the true quantum limit in just the direction which the observations show. Such a superposition resulting in the shift, $\Delta z$, is illustrated in the lower curves of Fig. 13. It turns out that if we assume the postulated overshoot, $z_{h}$, expressed in volts to be independent of the voltage at which the experiment is performed, then to explain the large shift (of order 4 or $5 \mathrm{v}$ ) observed at $24,500 \mathrm{v}$, we must assume $z_{h}$ so large that a vestige of the precipice would almost cer- 


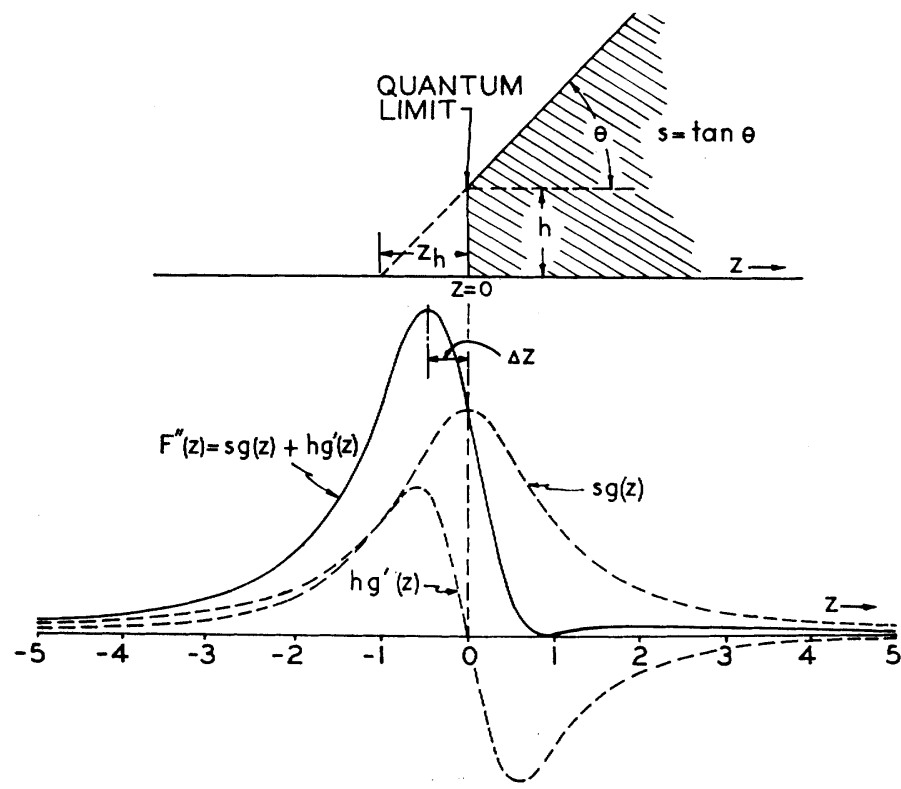

Fig. 13-Postulated precipice at the foot of the continuous X-ray spectrum which might account for the discrepancy discussed in the text.

tainly start to manifest itself in the isochromats taken at the lowest voltages. At these lowest voltages the monochromator pass bandwidths (in volts) are narrow enough so that one should see a rounded hump (convex upward) on the isochromat corresponding to the brink of the precipice in the true spectrum. Nothing like this, however, has been reported.

If then we are to explain the observed systematic trend of the results by the postulated precipice, it is necessary to assume that the overshoot, $z_{h}$, expressed in volts decreases with decreasing voltage. This is, however, not at all implausible since another feature, the position of the Ohlin irregularities, is known to be voltage dependent; its distance expressed in volts from the quantum limit decreases with decreasing voltage. The available data are insufficiently precise to yield information on the overshoot, $\Delta z$, as a function of voltage, which can be safely used to correct the experimental data. A more complete analysis of the theory of the continuous X-ray spectrum from thick targets is much needed.

Unfortunately there is little hope of improving the spectral resolving power in this experiment greatly beyond what already has been attained since the present results are already close to the theoretically attainable limit with perfect crystals according to the "dynamical theory" of selective X-ray reflection.

The conclusions to be drawn seem to be:

1) The most important residual source of systematic error in the short wavelength limit experiment comes from uncertainties, because of limited resolving power, as to how the true limit position should be estimated from the isochromat profile.

2) The X-ray isochromats obtained at the lowest voltages seem more likely to yield the most reliable results.

The nine determinations which were made at the lowest voltages (between 6000 and $11,000 \mathrm{v}$ ) form a cluster which is rather widely separated from the results obtained at the two higher voltages. In view of our ignorance of how best to correct for the above-described systematic trend, we shall adopt the weighted mean of this group of nine results made at the lowest voltages. Table VI presents these data which result in a weighted

TABLE VI

Selection of Data on Voltage-Wavelength Conversion Factor

The determinations made at the lowest voltages seem less likely to be in error from the systematic shift which we have ascribed to the postulated precipice at the quantum limit. The nine lowest voltage precision determinations are given below.

\begin{tabular}{l|c|c|c|c}
\hline Experimenter & $\begin{array}{c}\text { Nominal } \\
\text { Voltage }\end{array}$ & $\begin{array}{c}h c^{2} / \mathrm{e \Lambda} \\
(\mathrm{kv} \mathrm{X} \text { units })\end{array}$ & $\begin{array}{c}\text { Number of } \\
\text { Observations }\end{array}$ & Weight \\
\hline BJW $^{1}$ & 6112 & 12370.8 & 1 & 1 \\
$\mathrm{BS}^{2}$ & 8050 & 12371.9 & 1 & 1 \\
$\mathrm{BS}^{2}$ & 8050 & 12371.0 & 3 & 3 \\
$\mathrm{BS}^{2}$ & 8050 & 12370.1 & 1 & 1 \\
$\mathrm{BS}^{2}$ & 9860 & 12370.1 & 2 & 2 \\
$\mathrm{BJW}^{1}$ & 10186 & 12371.2 & 1 & 1 \\
\hline
\end{tabular}

Weighted mean value: $(12370.8 \pm 0.2) \mathrm{kv} \mathrm{X}$ units (S.D. by ex-

DuMond and Cohen ternal consistency)

1953 adjusted value: $(12372.2 \pm 0.4)$

1,2 See Table V.

mean value of the voltage-wavelength conversion factor

$12370.8 \pm 0.2$ (standard deviation by external consistency).

This still differs from the least-squares adjusted value of 1953

$$
12372.2 \pm 0.4 \mathrm{kv} \mathrm{X} \text { units }
$$

by an uncomfortably large amount. These two numerical values are not statistically independent so that care must be exercised in interpreting the significance of their disagreement. My associate, Dr. Cohen, has devised a useful method of assessing the seriousness of such a discrepancy between an input quantity and its adjusted output value in a least-squares adjustment and I plan to discuss this if time permits.

\section{PART II}

\section{Input Data Treated as Exact}

\section{9. $M_{a}$, Atomic Weights by Method of Nuclear Reaction} Energies and Mass Spectroscopy

In a nuclear reaction between a bombarding particle, $x$, and a target nucleus, $X$,

$$
X+x \rightarrow Y+y,
$$

a final particle, $y$, with kinetic energy, $T_{y}$, and a final nucleus, $Y$, with kinetic energy, $T_{Y}$, are produced. Conservation of energy requires that the total of the kinetic and rest-mass energies before and after the reaction shall be equal.

$$
\begin{aligned}
& \left(T_{x}+m_{x} c^{2}\right)+\left(M_{X} c^{2}\right) \\
& \quad=\left(T_{Y}+M_{Y} c^{2}\right)+\left(T_{y}+m_{y} c^{2}\right)
\end{aligned}
$$


The net gain in kinetic energy of the product particles above that of the input reactant particles, called the " $Q$ " of the reaction, is measured.

$Q=T_{Y}+Y_{y}-T_{x}=\left(M_{X}+m_{x}-M_{Y}-m_{y}\right) c^{2}$.

More than one nuclear reaction is needed to yield enough simultaneous equations such as (32), to obtain the mass difference between a pair of particles, as, for example, the neutron and the proton. However, a great number of nuclear reactions in the light elements has been precisely measured; so many, in fact, that the available information on nuclear $Q$ values affords a high degree of overdetermination for establishing mass differences of all the isotopes of all the light elements from the neutron through sulphur $(Z=16)$. Since this includes $\mathrm{O}^{16}$, the isotope which by definition has atomic mass exactly 16 on the physical scale, the method affords an extremely precise set of atomic mass values good to a few parts in a million for all these light nuclei. This corresponds to determining the $Q$ values of the reactions with an accuracy of the order of $1 \mathrm{kv}$. Li, Whaling, Fowler, and Lauritsen [50], in an important study in 1951, used the $Q$ values of 57 different nuclear reactions to establish a table of masses of 31 isotopes between $Z=0$ (the neutron) and $Z=9$ (fluorine). Van Patter and Whaling [51] in 1954 list separately all the $Q$-value determinations that have been made on no less than 474 nuclear reactions. About 600 independent $Q$ determinations are tabulated by them. A careful analysis of the mass data from nuclear reactions and a comparison of this with the mass-spectroscopic values has been made by Mattauch. ${ }^{15}$ To the accuracy here needed no evidence has appeared significantly discordant with the mass values and their error measures as given by $\mathrm{Li}$, Whaling, Fowler, and Lauritsen, though some work claiming slightly smaller error measures has recently been published by Wapstra [52]. The precision of the mass values given by $\mathrm{Li}$, Whaling, Fowler, and Lauritsen is amply sufficient for the purpose we shall make of them here in determining the general constants of physics. We, therefore, adopt the values given in Table VII

TABLE VII

Atomic Masses by Measurement of Nuclear Reaction Energies

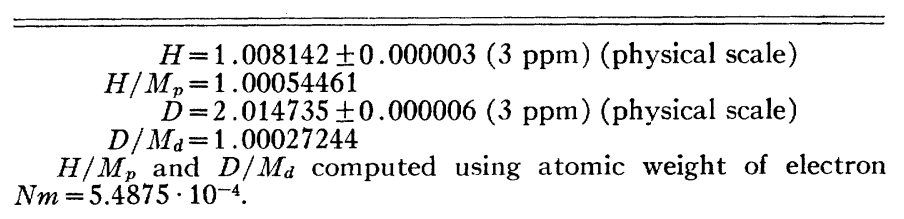

$N m$ may be obtained from the isotopic shift in the spectra of $H$ and $D$, the effect called by Sommerfield the "Mitbewegung des Kerns." It can also be determined by comparison of the cyclotron frequencies of electron and proton.

15 This first appeared in the minutes of the Avogadro Memorial Conference, Turin, Italy (Nuovo Cim., suppl., 1957). A still more complete and up-to-date treatment by F. Mattauch, Mainz, Ger., recently has been circulated privately. which are based on the work of these authors combined with $N m$, the atomic mass of the electron, which is required in order to compute $M_{p}$ and $M_{D}$, the atomic masses of the proton and the deuteron.

Atomic mass of electron, $\mathrm{Nm}$

$$
=0.000549 \text { (physical scale) } \text {. }
$$

Information on the atomic mass of the electron is available from two independent sources [53]-[56], 1) the isotopic shift in the spectrum of hydrogen and deuterium caused by the effect known in German as the "mitbewegung des kerns," and 2) the comparison of the "cyclotron frequency" of the electron with the "cyclotron frequency" of the proton. ${ }^{16}$

\section{0. $R_{\infty}$, Rydberg Constant for Infinite Mass (Taking Ac- count of Lamb Shift)}

Cohen [53] has re-analyzed the spectroscopic data of Houston [57], of Drinkwater, Richardson, and Williams [58], and of Chu [59], in the light of our present knowledge of the "Lamb shift," and has obtained

$$
R_{\infty}=109737.309 \pm 0.012 \mathrm{~cm}^{-1}( \pm 0.11 \mathrm{ppm}) .
$$

The Rydberg is an auxiliary constant which plays a role of great importance since it determines with great accuracy an important function of two of our unknowns, $\alpha$ and $e$.

$$
R_{\infty}=2 \pi^{2} m e^{4} h^{-3} c^{-1}
$$

and since

$$
h=2 \pi e^{2} /(\alpha c)
$$

we have

$$
R_{\infty}=m c^{2} \alpha^{3} /\left(4 \pi e^{2}\right) .
$$

Once the adjusted values of $\alpha$ and $e$ have been determined, we can compute Planck's constant, $h$, and the electron mass, $m$, from

$$
\begin{aligned}
h & =2 \pi e^{2} /(\alpha c) \\
m & =4 \pi e^{2} R_{\infty} \alpha^{-3} c^{-2} .
\end{aligned}
$$

\section{1. $\mu_{e} / \mu_{p}{ }^{\prime}$, Ratio of Electron Magnetic Moment to Proton Magnetic Moment}

(a) Methods of Gardner and Purcell [54], [55], Franken and Liebes [73]: I have described the measurement of the proton magnetic moment in terms of the nuclear magneton by comparison of the proton cyclotron frequency with the proton magnetic resonance frequency

16 A third less direct source of information has been pointed out by Huntoon and McNish in their contribution to the Avogadro Memorial Conference, Turin, Italy (Nuovo Cim., suppl., 1957). This is the work of Lindstrom, Ark. Fysik, vol. 4, p. 68; 1952. Lindstrom measured the radius of curvature in a magnetic field of internally converted $K$ and $L$ electrons from $T h B$, the $K-L$ energy difference being known from X-ray data. The proton nuclear resonance frequency in the same field was also measured. This experiment of course involves $\Lambda$. Lindstrom's result is said to be in surprisingly good agreement with Gardner and Purcell's, if $\Lambda=1.00202$ is used! 
in the same magnetic field. At Harvard University a similar experiment has been performed on the cyclotron frequency of the electron compared with the proton magnetic resonance frequency. This yields the proton magnetic moment in terms of the Bohr magneton.

The cyclotron frequency, $\omega_{e}$, of a free electron in a magnetic field, $B$, is given by

$$
\omega_{e}=e B /(m c) \quad(e \text { expressed in esu }) .
$$

The proton precession frequency, $\omega_{p}$, is given by

$\omega_{p}=\gamma_{p}{ }^{\prime} B=2 \mu_{p}{ }^{\prime} B / \hbar\left(\mu_{p}=\right.$ proton magnetic moment $)$

where $\gamma_{p}^{\prime}$ is the gyromagnetic ratio of the proton (uncorrected for diamagnetism), and $\mu_{p}^{\prime}$ is the proton magnetic moment (similarly uncorrected). The ratio $\omega_{p} / \omega_{e}$ is, therefore,

$$
\begin{aligned}
\omega_{p} / \omega_{e}=2 \mu_{p}{ }^{\prime} m c /(e \hbar)=\mu_{p}{ }^{\prime} / \mu_{0} ; \quad \mu_{0} & =\text { Bohr magneton. } \\
& =e \hbar /(2 m c) .
\end{aligned}
$$

This ratio is seen to be $\mu_{p}{ }^{\prime}$, the magnetic moment of the proton, expressed in Bohr magnetons, where the Bohr magneton is $\mu_{0}=e \hbar /(2 m c)$, a fundamental constant. To obtain $\mu_{p}{ }^{\prime} / \mu_{e}$ from these results we must know $\mu_{e} / \mu_{0}$, the ("anomalous") ratio of the electron magnetic moment to the Bohr magneton which modern quantumelectrodynamical theory has shown to differ slightly from unity. Until quite recently theory gave for this

$$
\begin{aligned}
\mu_{e} / \mu_{0} & =\left(1+\alpha / 2 \pi-2.973 \alpha^{2} / \pi^{2}\right) \\
& \left.=1.00114536 \text { (if } \alpha^{-1}=137.037\right) .{ }^{17}
\end{aligned}
$$

Very recently, however, Petermann at CERN has shown [72] that the original calculations of Karplus and Kroll [43] for the coefficient of the fourth-order term were in error and Karplus, and also Sommerfield, a student of $\mathrm{J}$. Schwinger, verified this. As a result, $\mu_{e} / \mu_{0}$ is about $14 \mathrm{ppm}$ larger than the above value.

If we combine the results of this experiment on $\omega_{p} / \omega_{e}$ with a knowledge of $\gamma_{p}{ }^{\prime}$, from the aforementioned work of Thomas, Driscoll, and Hipple [30] our first two equations, (35) and (36), show that we can calculate $e /(m c)$ the charge-to-mass ratio of the electron (expressed in emu) from the relation

$$
\omega_{p} /\left(\omega_{e} \gamma_{p}{ }^{\prime}\right)=e /(m c)
$$

Furthermore, if we combine $\omega_{p} / \omega_{e}$ from the results of this experiment with $\omega_{c} / \omega_{p}$ from the results of the Sommer, Thomas, and Hipple's [56] experiment described, we can obtain $\omega_{e} / \omega_{c}$, the ratio of the respective cyclotron frequencies of electron and proton, and this gives directly $m_{p} / m$, the ratio of the mass of the proton to the mass of the electron

$$
\omega_{e} / \omega_{c}=\left(\omega_{e} / \omega_{p}\right) /\left(\omega_{c} / \omega_{p}\right)=m_{p} / m .
$$

17 This value of $\alpha$ is practically beyond question. Our leastsquares analysis yields $\alpha^{-1}=127.0373 \pm 0.0006$. A change of 0.01 in $\alpha^{-1}$ produces a change of less than $10^{-7}$ in $\mu_{e} / \mu_{0}$.
The cyclotron frequency of the electron was measured by providing slits, $2 \mathrm{~mm} \times 0.1 \mathrm{~mm}$, on opposite sides of a rectangular shorted waveguide of $1 \times 0.5$-inch internal cross section and supplied with microwaves of frequency $9400 \mathrm{mc} \mathrm{sec}^{-1}$ in the $\mathrm{TE}_{10}$ mode so that a ribbon-shaped beam of slow electrons drifting across the one-inch dimension of the guide from one slit to the other crossed in a region of voltage maximum of the microwaves. The electrons were supplied by an oxidecoated cathode in a copper cavity behind one of the slits and were collected by an electrode in a second copper cavity behind the other slit. This equipment was placed in a magnetic field of 3300 gauss, between the poles of an electromagnet with the direction of the field parallel to the general direction of motion of the electrons from slit-to-slit across the waveguide. Closely adjacent to the waveguide between the magnet poles was placed the proton resonance head. This operated on a frequency of $14.24 \mathrm{mc} \mathrm{sec}^{-1}$. This frequency was multiplied 657 times for comparison with the electron cyclotron frequency in the waveguide. The positions of proton resonance head and waveguide between the magnet poles could be interchanged so that by averaging the results before and after such interchange the effect of any slight difference in magnetic field intensity at these two elements could be compensated.

The electrons drift in helical paths from one slit to the other across the waveguide executing the turns of their helices at the cyclotron frequency, $\omega_{e}=e B /(\mathrm{mc})$. If this frequency matches exactly the microwave frequency of the electric vector in the waveguide, the electrons gain energy at each turn and their helical trajectories will expand so that many of them will fail to pass through the second slit. It was thus anticipated that resonance would be recognized by a decrease in current to the collector (see Fig. 14). For every weak electron accelerating voltage, $-V_{a}$, at the cathode, however, space-charge effects produced a current peak at resonance. ${ }^{18}$

${ }^{18}$ To make the resonance as sharply defined as possible it is desirable to have very slow electrons so that a maximum number of cyclotron cycles will be executed during the transit time from slit-toslit in the region of the radio-frequency field. The cathode was held more negative than the waveguide by a voltage, $V_{a}$, adjustable from 0 to $5 \mathrm{v}$, and the collector was maintained approximately $20 \mathrm{v}$ positive relative to the guide so as to collect all the electrons passing through the second slit. When $V_{a}$ was approximately $5 \mathrm{v}$ the expected dip in anode current (from approximately 0.4 to $0.3 \mu \mathrm{a}$ ) was observed at resonance. However, it was found that peaks instead of dips occurred at resonance, if $V_{a}$ was less than about $3 \mathrm{v}$. The peaks were considerably sharper than the dips, having a width of order 0.5 gauss contrasted with 5 gauss for the dips. The anode current under these circumstances was less than $0.0005 \mu \mathrm{a}$ when away from resonance. This unexpected behavior was attributed to space-charge limitation of the electron current through the guide save at resonance. The electrons having the slowest transverse velocity across the guide would be the ones most responsible for this space-charge limitation, and these are also the ones which at resonance are acted upon for the longest time by the microwaves. The resulting expansion of their orbits when resonance obtains would reduce the space-charge density and thus permit an increase in current across the guide on the part of other electrons (presumably those directed most nearly parallel to the magnetic field). Because of its superior sharpness, the peak attributed to the suppression of space-charge limitation was the one exclusively used for the measurements. 
J. H. Gardner and E. M. Purcell,

Phys. Rev. 76, 1262 (19,49)

J. H. Gardner,

Phys. Rev. 83, 996 (1951)

The electrons drift in helical paths in microwave field in wave guide executing turns at the cyclotron frequency,

$$
\omega_{e}=\mathrm{e} B /(m e)
$$

If $\omega_{e}$ matches exactly the microwave input frequency the electrons gain energy and miss the second slit and a dip in current would indicate resonance. For very low cathode voltage, $-\mathrm{V}_{\mathrm{a}}$, however, space-charge effects produced a current peak at resonance.

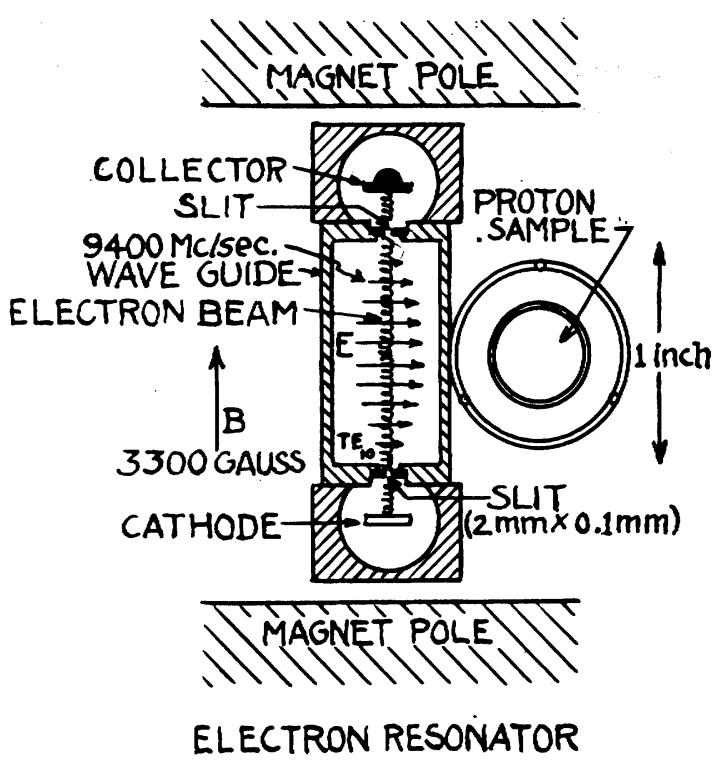

Results:--

$$
\begin{aligned}
& \omega_{e} / \omega_{p}=\mu_{o} / \mu_{p}^{\prime}=657.475 \pm 0.008 \quad \text { (without diamagnetic correction) } \\
& \omega_{e} / \omega_{c}=m_{p} / m=1836.12 \pm 0.03
\end{aligned}
$$

Fig. 14-Determination of the ratio, proton resonance frequency to electron cyclotron frequency, $\omega_{p} / \omega_{e}$, by the method of Gardner and Purcell.

The final value assigned by Gardner [55] was

$$
\mu_{0} / \mu_{p}{ }^{\prime}=\omega_{e} / \omega_{p}=657.475 \pm 0.008
$$

without the diamagnetic correction to the field of the proton for the electrons in the hydrogen molecule. If we calculate $\omega_{e} / \omega_{c}$ by (40) using $\omega_{e} / \omega_{p}$ (41) and the results of Sommer, Thomas, and Hipple [56], for $\omega_{c} / \omega_{p}$, one obtains for the ratio of the rest masses of proton and electron

$$
\omega_{e} / \omega_{c}=m_{p} / m=1836.12 \pm 0.03 \text {. }
$$

Franken and Liebes [73] have pointed out the possible need for a correction to this result because, at highcurrent density, space charge might exert a radial force on the electronic orbits in addition to the magnetic force, $v \times \mathrm{H}$. They have performed an experiment in which this correction is made.

(b) Method of Koenig, Prodell, and Kusch [60]: A quantity which is closely related to the one measured by Gardner and Purcell [54] is the ratio, $\mu_{e} / \mu_{p}{ }^{\prime}$, of the electron magnetic moment to the proton magnetic moment (recall that we are using $\mu_{p}{ }^{\prime}$ for the apparent moment before correction for the diamagnetic effect; $\mu_{p}$ is the corrected moment). This ratio was measured by Koenig, Prodell, and Kusch [60] at Columbia University with such high accuracy (an error of \pm 0.6 ppm) that it can be very safely treated as a fixed auxil- iary constant among the input data of our present leastsquares analysis (see Fig. 15). The well-known molecular beam method [61] for measuring nuclear magnetic moments was used in this measurement. An atomic beam passes successively through three magnetic fields: the first and last, (the " $A$ " and " $B$ " fields), are inhomogeneous with gradients in opposite directions, while the intermediate field is uniform. Because of the interaction of the atomic dipole moment and the inhomogeneous magnetic field, an atom will suffer a deflection in passing through the " $\mathrm{A}$ " field. The " $\mathrm{B}$ " field then impresses on the atom a deflection in the opposite direction. Whatever the magnetic moment of the atom may be (as long as it is the same in both fields), the two deflections will compensate each other, and the atom will return to the instrument axis after passing through the two fields. If, however, during the time of transit through the intemediate "C" field, a transition is induced in the atom so that its magnetic dipole moment is altered, such atoms will not receive the correct compensating deflection in the " $\mathrm{B}$ " field and will fail to pass through the exit slit of the instrument. A magnetic field of frequency $3655 \mathrm{mc} \mathrm{sec}^{-1}$ perpendicular to the direction of the static field was used to induce the transitions between hyperfine structure levels in hydrogen in ${ }^{2} S_{1 / 2}$ state. The transition frequency of the line $(1,0 \leftrightarrow 1,-1)$ was measured and from this, and 
S. H. Koenig, A. G. Prodell and P. Kusch (Phys. Rev. 88, 191 (1952)) used the well-known method of Rabi, Millman, Kusch, and Zacharias.

A beam of H-atoms is deflected downward in the A-field and upward in the B-field by an equal amount unless the magmetic moment of the atoms is changed by the R.F. present in the C-field, in which case the atoms miss the final slit.

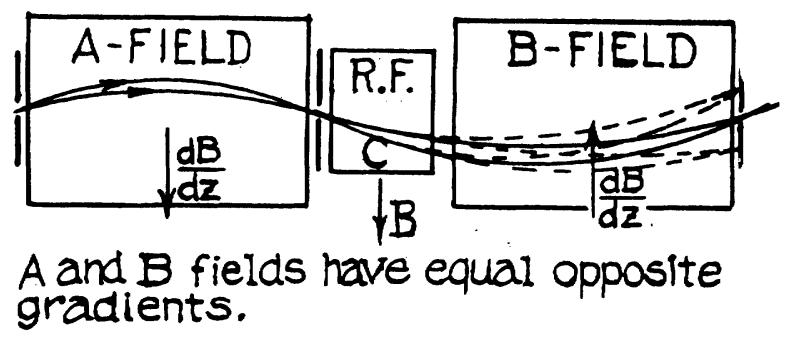

$3655 \mathrm{mc} / \mathrm{sec}$ R.F. used to induce hyperfine structure transitions. From the transition frequency of the line $(1,0 \leftrightarrow 1,-1)$ in the field B together with the proton nuclear magnetic resonance frequency for the same field $B$, the ratio, $g_{p} / g_{j}$, of the proton $g$-value to the g-value for the hydrogen atom in the ${ }^{2} \mathrm{~S}_{1 / 2}$ state, the latter closely related to the electron spin g-value, $\mathrm{g}_{\mathbf{s}}$, was measured with the result:--

$$
g_{s} / g_{p}=M_{p} /\left(N m \mu^{\prime}\right) \quad\left(1+a / 2 \pi-2.973 \alpha^{2} / \pi^{2}\right)=658.2288 \pm 0.0004
$$

\footnotetext{
Here $M_{p}=$ atomic wt. of proton, $\mathrm{Nm}=$ atomic wt. of electron, $\mu^{\prime}=$ mag. mom. of proton (uncorrected for diamag.) expressed in nuclear magnetons and the trinomial is $\mu_{e} / \mu_{0}$, the electron magnetic anomaly ratio, 1.00114536 .
}

From this $\mu_{0} / \mu_{p}^{\prime}=M_{p} /\left(N m \mu^{\prime}\right)=657.4758 \pm 0.0004$

Fig. 15-Ratio electron magnetic moment to proton magnetic moment, $\mu_{e} / \mu_{p}{ }^{\prime}$ by the atomic beam method of Koenig, Prodell, and Kusch.

measurements of the proton nuclear magnetic resonance frequency in the same magnetic field (Thomas, Driscoll, and Hipple), $g_{p} / g_{j}$ could be calculated. $g_{p}$ is the proton $g$ value while $g_{j}$, the $g$ value for the hydrogen atom in the ${ }^{2} S_{1 / 2}$ state, is closely related to the electron spin $g$ value, $g_{s}$. The experimental result obtained by Koenig, Prodell, and Kusch was (before the correction for diamagnetism in the proton resonance oil sample),

$$
\begin{aligned}
g_{s} / g_{p} & =\left[M_{p} /\left(N m \mu^{\prime}\right)\right]\left(1+\alpha / 2 \pi-2.973 \alpha^{2} / \pi^{2}\right) \\
& =658.2288 \pm 0.0004
\end{aligned}
$$

in which $M_{p}$ is the atomic weight of the proton, $N$ is Avogadro's number, $m$ the mass of the electron, $\mu^{\prime}$ the magnetic moment of the proton (uncorrected for diamagnetism) expressed in nuclear magnetons, and the trinomial in the parenthesis is $\mu_{e} / \mu_{0}$, the correction factor for the anomalous magnetic moment of the electron [42], [43]. This can be computed from the value $\alpha^{-1}=137.037$, with ample accuracy [see (38)] to place $\mu_{e} / \mu_{0}$ in the category of auxiliary fixed constants. The result is

$$
\mu_{0} / \mu_{p}{ }^{\prime}=M_{p} /\left(N m \mu^{\prime}\right)=657.4758 \pm 0.0004 .
$$

Unfortunately, this calculation is vitiated to the extent of about 14 parts per million because of the error subsequently discovered in Kroll's original theoretically calculated numerical coefficient, -2.973 in (43) which we now know should be -0.328 .

(c) Resonant Cavity Method of Beringer and Heald [26]: Beringer and Heald have measured the ratio of proton moment-to-electron-moment, the same ratio as that of Koenig, Prodell, and Kusch, using a microwave absorption technique (see Fig. 16.) Since this method is basically distinct from the molecular beam method used by Koenig, Prodell, and Kusch, a comparison of the two results is useful. Hydrogen atoms are contained in a resonant cavity placed in a uniform magnetic field. This field produces a Zeeman splitting of the atomic energy levels and the strength of this field is varied until the Zeeman splitting corresponds to the resonant frequency of the cavity. The absorption of energy by the atoms at this resonance reduces the energy transmitted through the cavity from a klystron oscillator to a detector. The magnetic field in the experiment is measured by a proton resonance probe. Thus the magnetic moment of the proton and the electron are directly 


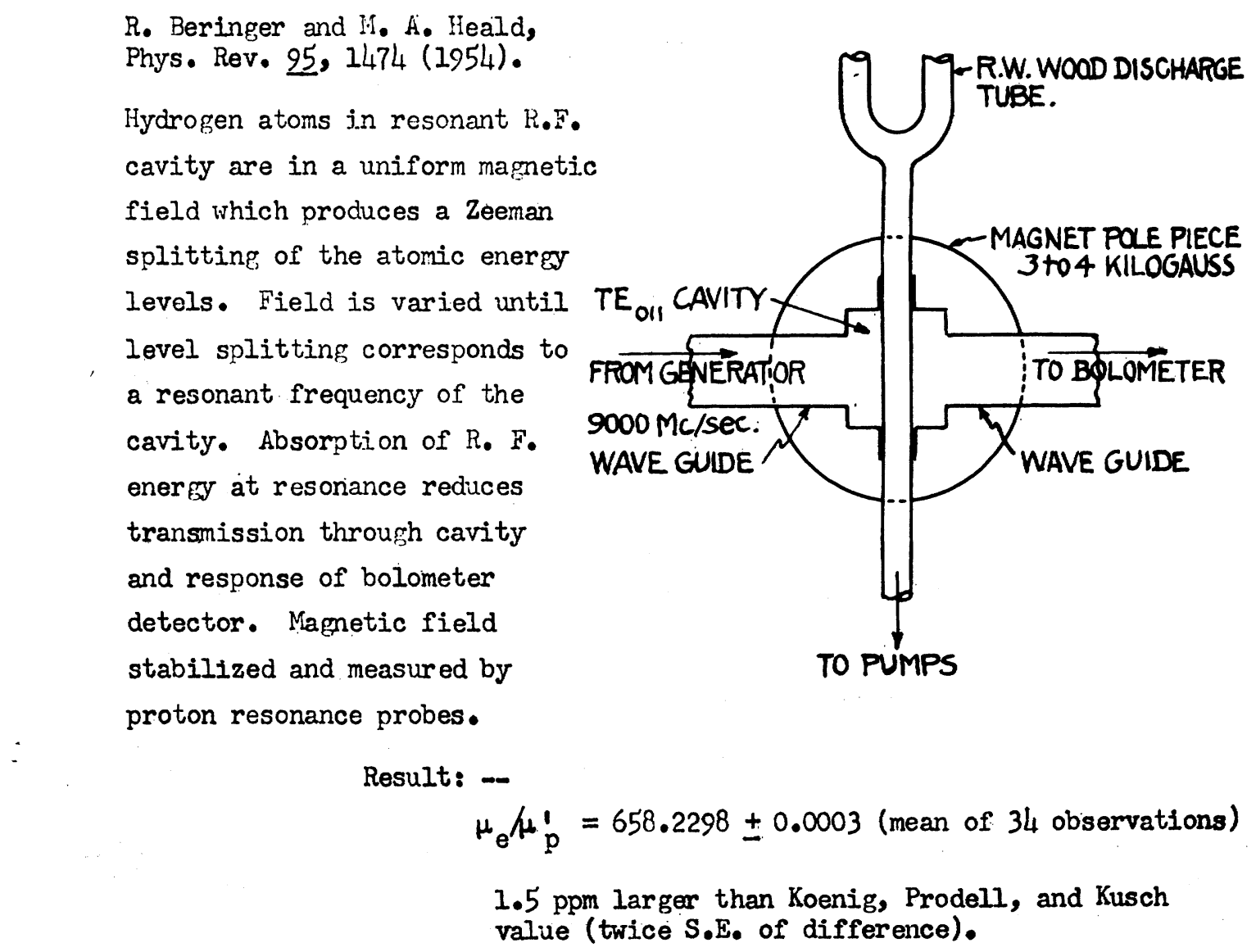

Fig. 16-Ratio, electron magnetic moment to proton magnetic moment, $\mu_{e} / \mu_{p}{ }^{\prime}$ by the resonant cavity method of Beringer and Heald.

compared. The result, uncorrected for diamagnetism in a spherical sample of mineral oil, is

$$
\mu_{e} / \mu_{p}{ }^{\prime}=658.2298 \pm 0.0003
$$

where the quoted error is a statistical standard deviation of the mean of 34 observations after 10 discordant data were rejected. This result is $1.5 \mathrm{ppm}$ larger than Koenig, Prodell, and Kusch's measurement (43). The disagreement, though small relative to the precision of most other measurements, is twice as large as the standard error of the difference computed from the quoted errors for these two.

In obtaining the result of Koenig, Prodell, and Kusch, (44), to compare with the above measurement of Gardner and Purcell, we made use of the theoretical value of the anomalous moment of the electron (38). We can also reverse the procedure and calculate the anomalous electron moment by combining Gardner and Purcell's results with the measurements of Koenig, Prodell, and Kusch, or Beringer and Heald. In either case we obtain:

$\mu_{e} / \mu_{0}=1.001147 \pm 0.000008$ (experimental value),

where the error is entirely due to the Gardner and Purcell value and masks the difference between the two values of $\mu_{e} / \mu_{p}{ }^{\prime}$, (those of Koenig, Prodell, and Kusch, and of Beringer and Heald). The agreement between this experimental value of $\mu_{e} / \mu_{0}$ and the older theoretical value 1.00114536 was excellent and it serves as a verification of the validity of the new quantum electro- dynamics, but after correction for the recently discovered Karplus error of $14 \mathrm{ppm}$ the new theoretical value, $\mu_{e} / \mu_{0}=1.001159$ differs by $12 \mathrm{ppm}$ from the observed value. This was one of the motivations for the repetition of the Gardner-Purcell experiment by Franken and Liebes [73] who, as stated above, believe they have found a small source of systematic error from space charge.

\section{Recent Measurements of the Velocity of Light}

Table VIII shows the result of nine independent determinations of the velocity of light (or of radio waves) made since 1949. All these methods indicate quite unequivocally that the earlier weighted average value arrived at by Birge, based chiefly on earlier measurements by Michelson, Pease, and Pearson [63] with the rotating mirror method in an evacuated tube, and by Anderson [64] using Kerr-cell modulation, namely $299,776 \mathrm{~km} \mathrm{sec}^{-1}$, was low by about 16 or $17 \mathrm{~km}$ $\mathrm{sec}^{-1}$. The newer values (those after 1948) are not all of equal reliability or accuracy, nor are they all completely in agreement, and in particular the Hansen and Bol resonant cavity result [65] disagrees with the others by about $3 \mathrm{~km} \mathrm{sec}^{-1}$, a disagreement which, though small judged by earlier standards, is uncomfortably large relative to the claimed probable errors. A plausible explanation for this discrepancy on the basis of a thin surface layer of abnormally high resistivity caused by work- 
TABLE VIII

Recent Measurements of the Velocity of Light

\begin{tabular}{|c|c|c|c|c|}
\hline Author & Date & Method & $c$ in $\mathrm{km} \mathrm{s}^{-1}$ & Remarks \\
\hline $\begin{array}{l}\text { Aslakson } \\
\text { Hansen }^{1} \\
\text { Essen }^{3} \\
\text { Bergstrand }^{4} \mathrm{Bol}^{2} \\
\text { Froome }^{5} \\
\text { Mackenzie }^{6} \\
\text { Froome }^{5} \\
\text { Plyler, Blaine and Connor }^{7} \\
\text { Florman }^{8}\end{array}$ & $\begin{array}{l}1949 \\
1950 \\
1950 \\
1951 \\
1952 \\
1953 \\
1954 \\
1955 \\
1955\end{array}$ & $\begin{array}{l}\text { "Shoran" } \\
\text { FLCR } \\
\text { VLCR } \\
\text { Geodimeter } \\
\text { FSMWI } \\
\text { Geodimeter } \\
\text { FSMWI } \\
\text { IRSP } \\
\text { FSMWI }\end{array}$ & $\begin{array}{l}299,792 \pm 3.5 \\
299,789.3 \pm 1.2 \\
299,792.5 \pm 1.0 \\
299,793.1 \pm 0.32 \\
299,792.6 \pm 0.7 \\
299,792.4 \pm 0.5 \\
299,793.0 \pm 0.3 \\
299,792 \pm 6 \\
299,795.1 \pm 1.9\end{array}$ & $\begin{aligned} \text { FLCR } & =\text { fixed length cavity resonance } \\
\text { VLCR } & =\text { variable length cavity resonance } \\
\text { FSMWI } & =\text { free space microwave interferometer } \\
\text { IRSP } & =\text { infrared spectrometer }\end{aligned}$ \\
\hline
\end{tabular}

${ }^{1}$ C. I. Aslakson, Nature, vol. 164, p. $711 ; 1949$.

${ }^{2}$ K. Bol, Phys. Rev., vol. 80, p. 298; 1950.

${ }^{3}$ L. Essen, Proc. Roy. Soc., vol. A 204, p. 260; 1950.

${ }^{4}$ E. Bergstrand, Arkiv. f. Fys., vol. 2, p. 119; 1950.

5 K. D. Froome, Proc. Roy. Soc., vol. A 213, p. 123; 1952. Vol. A 223 , p. $195 ; 1954$

6 I. C. C. Mackenzie, Ord. Surv. Prof. Papers 19, H.M. Stat. Office; 1954.

7 E. K. Plyler, L. R. Blain, and W. S. Connor, J. Opt. Soc. Am., vol. 45, p. 102; 1955.

${ }^{8}$ E. F. Florman, NBS Tech. News Bull., vol. 39, p. $1 ; 1955$.

hardening incident to polishing has been suggested by Dayhoff. ${ }^{19}$

Of the determinations listed on this figure probably the most accurate and reliable are: 1) those of Bergstrand [66] in Norway and of Mackenzie [67] in Scotland, each using Kerr-cell modulated visible light over long light paths and employing Bergstrand's highly ingenious "Geodimeter"; and 2) those of Essen [68] and of Froome [69], Essen ${ }^{20}$ using microwave cavity resonance and Froome the free-space microwave interferometer. These results are all in excellent agreement.

I only shall have time to describe one of these methods, that of Froome, at the National Physical Laboratory, England.

The four-horn Fraunhofer diffraction microwave interferometer of Froome is shown schematically in Fig. 17. The source of microwaves is a Pound stabilized reflex klystron oscillator with a frequency of 24005 mc $\sec ^{-1}$ corresponding to a wavelength of about 1.25 $\mathrm{cm}$. The accuracy of the frequency measurement is about one part in $10^{8}$. Energy from the oscillator passes to a hybrid junction ("magic $\mathrm{T}$ ") which serves as a beam divider, from which it passes through two long waveguide arms to the pair of transmitting horns. The

19 In this experiment the true electrical diameter of the cavity is greater than the mechanical diameter by an amount of the order of magnitude of the skin depth of the walls, a depth which depends, of course, on the frequency of the particular mode of oscillation which is being excited. The possible presence of a film of silver sulphides of unknown thickness with a conductivity and a dielectric constant differing from the values for bulk silver will affect the observed resonant frequency of the cavity. Correction for this should increase the Hansen and Bol value of the velocity slightly. It has been suggested in a private communication by $E$. S. Dayhoff that the mechanical effect of polishing the silver-plated surface may cold-work the metal and thus greatly decrease its conductivity in an extremely shallow layer. It is unfortunate that Hansen and Bol only made measurements at just enough frequencies to determine $c$ in the absence of such anomalous skin effects. In any precision measurement it is always a better policy to overdetermine the measurements in an effort to uncover unsuspected systematic errors.

20 In a recent communication Essen informs me that the estimated standard deviation of $\pm 1 \mathrm{~km} \mathrm{sec}^{-1}$ in his 1950 determination contained an allowance for possible systematic error and that the standard deviation computed from only the statistical variance of his data would be nearer $\pm 0.3 \mathrm{~km} \mathrm{sec}^{-1}$. matching stub and phase shifter (1) to the left of the beam divider, together with a "constant phase auxiliary interferometer" (cpi) constitute a device ${ }^{21}$ for altering the amplitude of the energy transmitted down this arm without producing a phase displacement. The phase shifter (2) to the right of the beam divider, together with the variable attenuator, is required in order to adjust and balance the position of the first interference minimum.

The movable part of the interferometer situated between the transmitting horns consists of a pair of receiving horns mounted on a carriage constructed almost entirely of silica tubes (for thermal stability) and arranged to travel on ways through a path of about one meter. The two received signals are mixed to produce interference, and detected by means of a simple superheterodyne arrangement; the output is rectified and indicated on a millimeter. An interference minimum is then detected as minimum current through the meter, and the carriage can be set on a minimum to better than one micron.

To make a wavelength measurement the exact displacement of the carriage corresponding to 81 wavelengths (162 minima) is measured to one-quarter micron by means of end-contact gauges.

Froome stated that the $24005 \mathrm{mc} \mathrm{sec}^{-1}$ equipment is merely a prototype for the investigation of sources of inherent error. The conditions, therefore, were chosen deliberately to cause errors; as, for example, the random effects arising from reflections in the rather small room. Nevertheless, the apparatus was found to be capable of an accuracy of one part in $10^{6}$ for the velocity measurements. Much better results are anticipated, there-

21 This ingenious device splits the input wave into two components of equal amplitude in two different paths. These components are subsequently recombined after a change of phase brought about by an increase in the path length for one path and an exactly corresponding decrease in the path length for the other. The two equal vectors representing the phases of the two components are thus rotated in opposite directions through the same angle so that the direction of their resultant is unchanged while its amplitude is reduced. 


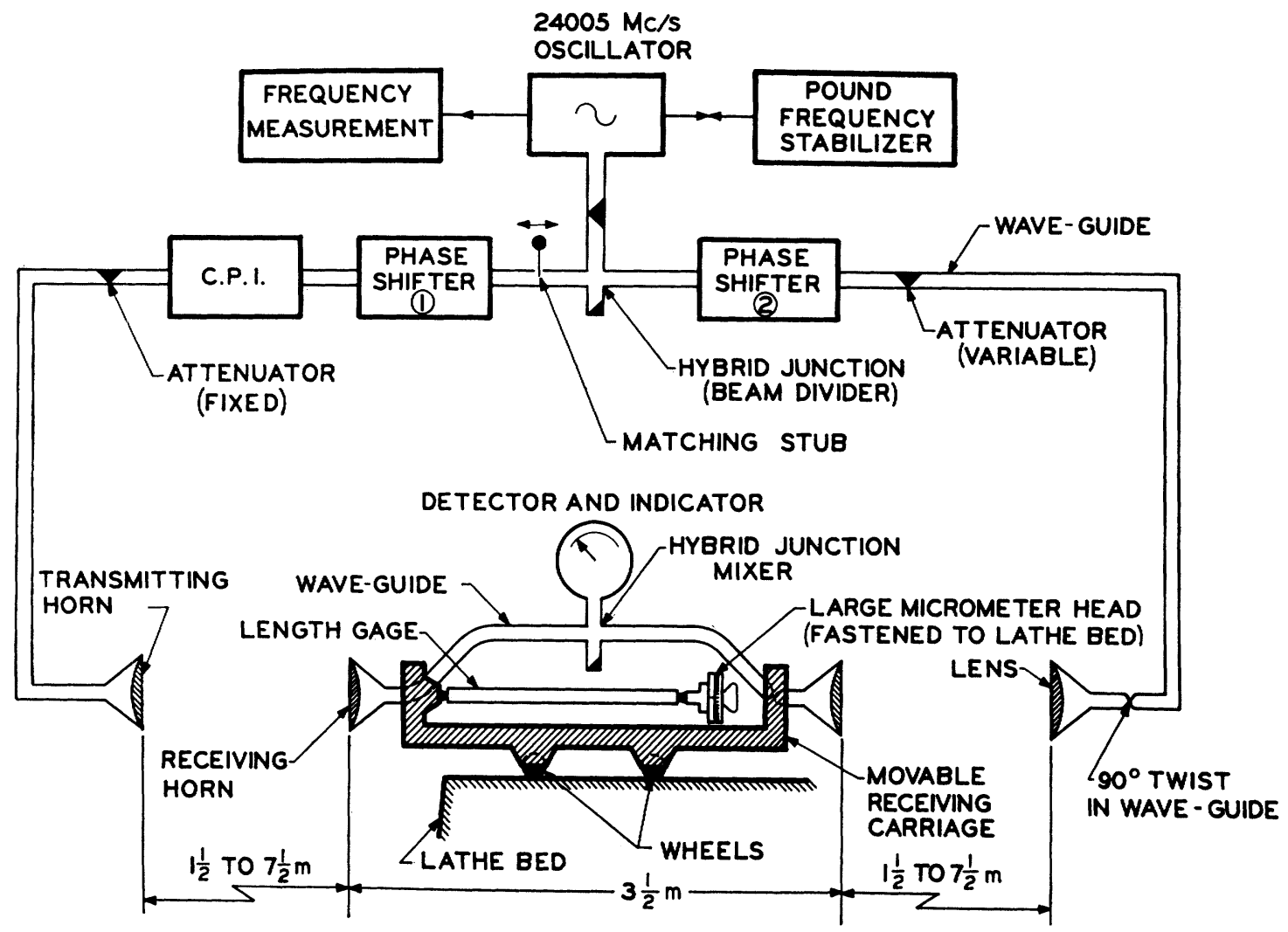

Fig. 17-Free space microwave interferometer of Froome.

fore, with the final equipment which is to operate at $70,000 \mathrm{mc} \mathrm{sec}^{-1}(\lambda=4 \mathrm{~mm})$ over a path difference of 1000 minima in a much larger room. Ten observations with the present prototype have been reduced to yield a preliminary values for the free-space velocity of electromagnetic waves in vacuo of

$$
c=299793.0 \pm 0.3 \mathrm{~km} \mathrm{sec}^{-1} .
$$

Space limitations preclude description of many details and refinements to be found in the original papers and in particular the study of corrections for refractive index of the air and water vapor given in two papers by Essen and Froome [70], [71].

Cohen and I have adopted the above value, $c=299793.0 \pm 0.3 \mathrm{~km} \mathrm{sec}^{-1}$, as one of our auxiliary constants in our most recent (1955) evaluation of the universal constants. Very recently the radio engineers and physicists have adopted a value, minutely lower, I believe, namely

$$
c=299792.9 .
$$

This change of $0.3 \mathrm{ppm}$ would have no significant effect on any of our output values. It is based on the most recent results of Froome [74] and of Bergstrand [75] which are now in highly satisfactory agreement.

Bergstrand, after taking into account his own work and that of others with his "Geodimeter" and also the results of Froome including the latter's most recent final determination, has arrived [75] at the following weighted mean which he considers the present "best" value.

$$
c=299792.7 \pm 0.2 \mathrm{~km} \mathrm{sec}^{-1} .
$$

Here again this change from our adopted value above would affect our values of the constants insignificantly.

Recent suggestions from several quarters that the velocity of light in vacuum may vary with frequency are very improbable from three considerations.

a) Eclipsing binary stars do not show colors at the time of the eclipse.

b) The "red shifts" of optical lines and of the radiofrequency line from the Lamb-doublet level in hydrogen have recently been observed for the radio object in Cygnus of the order of $10^{9}$ light years distant and have been shown to be identical.

c) At the California Institute of Technology we have measured [76] the wavelength, $h /(\mathrm{mc})$, of the $511-\mathrm{kv}$ annihilation radiation line from the recombination of $\mathrm{Cu}^{64}$ positrons and electrons in copper with the 2-meter bent quartz crystal diffraction spectrometer and have shown that it agrees to a part in $10^{4}$ with the predicted value.

This last is a test of the velocity, $c$, at very high frequencies indeed, twenty thousand times that of Bergstrand's visible light waves and forty billion times that of Froome's 1-cm waves.

\section{PART III}

\section{Critical Analysis of the Preceding Data} Using the Method of Least-Squares

13. The Overdetermined Nature of the Problem-A Geometrical Interpretation

The reader is referred to Table $\mathrm{I}$ at this point to remind him of the nature of the data we have assembled. 
Each of the quantities whose measurement I have described can be expressed in terms of $h$, Planck's constant, $e$, the electronic charge, $m$, the electron restmass, $N$, the Avogadro number, $\Lambda$, the $\mathrm{X}$-unit-milliangström conversion constant, $c$, the velocity of light, and a few other auxiliary quantities whose numerical values are accurately known. The Rydberg constant, however, which is a function of $h, e, m$, and $c$, is much more accurately measured than any of its component factors. It is, therefore, convenient to consider the Rydberg constant as an exactly known numerical quantity and to use it to express the electron mass as a known function of the other variables. Also, to the accuracy required here, it is convenient and justifiable to treat the velocity of light, $c$, as an exactly known quantity. Furthermore, because of the fact that the fine structure constant, $\alpha$, is more accurately determined (by the work of Lamb and his coworkers [38]-[41]) than it can be computed by combining, say measurements of $e, h / e$, and $c$, it is also convenient to use $\alpha$ as a variable in place of $h$. Thus we shall express all of the experimental results in terms of the four quantities $\alpha, e, N$, and $\Lambda$, which we shall call the primary unknowns of our analysis. After effecting the eliminations and substitutions just described, our data yield the seven kinds of equations of Table I, comprising a total of thirteen equations in four unknowns. These we shall call our primitive equations. These clearly constitute a considerably overdetermined set. Since the numerical data are the results of experimental measurements subject to possible errors of both systematic and random nature, it is too much to expect the set to be exactly compatible. We can only hope that the incompatibilities are of the order of magnitude consistent with the estimated precision of the measurements, and if this is not the case, we must try to ferret out the systematically erroneous data and eliminate them or, if possible, correct them. A situation of this sort calls for a critical analysis by least-squares.

My time does not permit me to give a rigorous derivation or a carefully reasoned defense of the doctrine of least-squares here. For such a discussion I must refer you to a really excellent treatment by my colleague and coworker, Cohen [77], in the proceedings of the Turin Conference of 1957. (For a still more complete exposition see the text by Cohen, Crowe, and DuMond [78], or the treatment by Cohen and DuMond, in Flugge's "Handbuch der Physik" [79].)

If we examine the seven kinds of equations in Table I we see that the result of any experiment can be described as measuring (except for quantities that can be considered as accurately calculable correction factors) some product of powers of the primary variables of the form:

$$
f(\alpha, e, N, \Lambda)=\alpha^{a} e^{b} N^{c} \Lambda^{d}=A(1 \pm \sigma) .
$$

The second expression is not actually an equation in the strictest sense. $A$ is the numerical result of a physical measurement and $\sigma$, its estimated or assigned standard deviation. The expression is, therefore, a short-hand method for indicating that we do not know the true value of the right-hand member, but that the experiment can be interpreted as indicating a value which is defined only to the extent of some probability distribution (not necessarily Gaussian) with mean value $A$ and with relative "standard deviation," i.e., root-meansquare deviation, $\sigma$.

We can now think of a four-dimensional "constants space" in which the four unknowns, $\alpha, e, N$, and $\Lambda$, regarded as continuous variables are represented on four orthogonal cartesian coordinates. Each functional relationship (each experiment of Table I) then is represented by a surface in this space. An arbitrary point in the space (a given set of values of the constants, say $\alpha$, $e, N, \Lambda)$ is in agreement with the experimental result if it lies in the surface. A point (that is a set of values of the constants) is, therefore, consistent with several experimental results if it lies in the common intersection of the surfaces. In general, if we have more experiments (surfaces or equations) than we have constants to be determined (dimensionality of the space), it cannot be guaranteed (in view of the liability of error) that there will be one point through which every surface passes. If the experiments are reasonably consistent there will be a point, however, which is close to all of the surfaces.

Varying the experimentally determined numeric, $A$, in each equation produces motion of the surface in the direction of its normal, and we can visualize the statistical distribution of which $A$ is the mean value and $\sigma^{2}$, the variance, by thinking of the surface not as infinitesimally thin but as endowed with a certain domain of fuzziness on either side of the mean value surface, $A$.

For convenience in analyzing the data we linearize our equations. We adopt origin values, $\alpha_{0}, e_{0}, N_{0}, \Lambda_{0}$, corresponding to a judiciously selected point in our space chosen sufficiently close to our expected solution that any set of values, $\alpha, e, N, \Lambda$, in which we are likely to be interested will differ from the origin values by small amounts. "Small" is determined here by the magnitudes of the second derivatives of the function, $f$; we assume that we can expand $f(\alpha, e, N, \Lambda)$ as a multivariate Taylor series about the origin point, and that in such an expression only the linear terms need be retained. In practice what we actually do is equivalent to expanding the logarithm of $f$ in a Taylor series; we thus deal with relative deviations, and if the function is a simple product of powers as is the case here, we obtain in place of (48) the linearized equation

$$
a x_{\alpha}+b x_{e}+c x_{N}+d x_{\Lambda}=h \pm \sigma,
$$

where $x_{\alpha}=\left(\alpha-\alpha_{0}\right) / \alpha_{0} ; x_{e}=\left(e-e_{0}\right) / e_{0}$ etc., and where $h=\left(A-A_{0}\right) / A_{0}$, in which $A_{0}$ is the value of the function, $f$, evaluated with the origin values $\alpha_{0}, e_{0}, N_{0}, \Lambda_{0}$. ( $h$, here is not to be confused with Planck's constant.) The $x$ 's are thus pure numbers, and it is frequently convenient to express them as parts per hundred thousand or parts per million (ppm) deviations from the origin values. (The largest deviation of any of our ad- 
TABLE IX

Data for Preliminary 1952 Adjustment

$f(\alpha, e, N, \Lambda)=\alpha^{a} e^{b} N^{c} \Lambda^{d}=A(1 \pm \sigma)$

Adopt consistent origin values, $\alpha_{0}, e_{0}, N_{0}, \Lambda_{0}, A_{0}$ close to the expected solution and define

$x_{\alpha}=\left(\alpha-\alpha_{0}\right) / \alpha_{0} ; x_{e}=\left(e-e_{0}\right) / e_{0}$ etc. and $H=\left(A-A_{0}\right) / A_{0}$ then (48) becomes linearized as:

$a x_{\alpha}+b x_{e}+c x_{N}+d x_{\Lambda}=h \pm \sigma$.

Complete Set of 13 Linearized Equations from Data of Table I

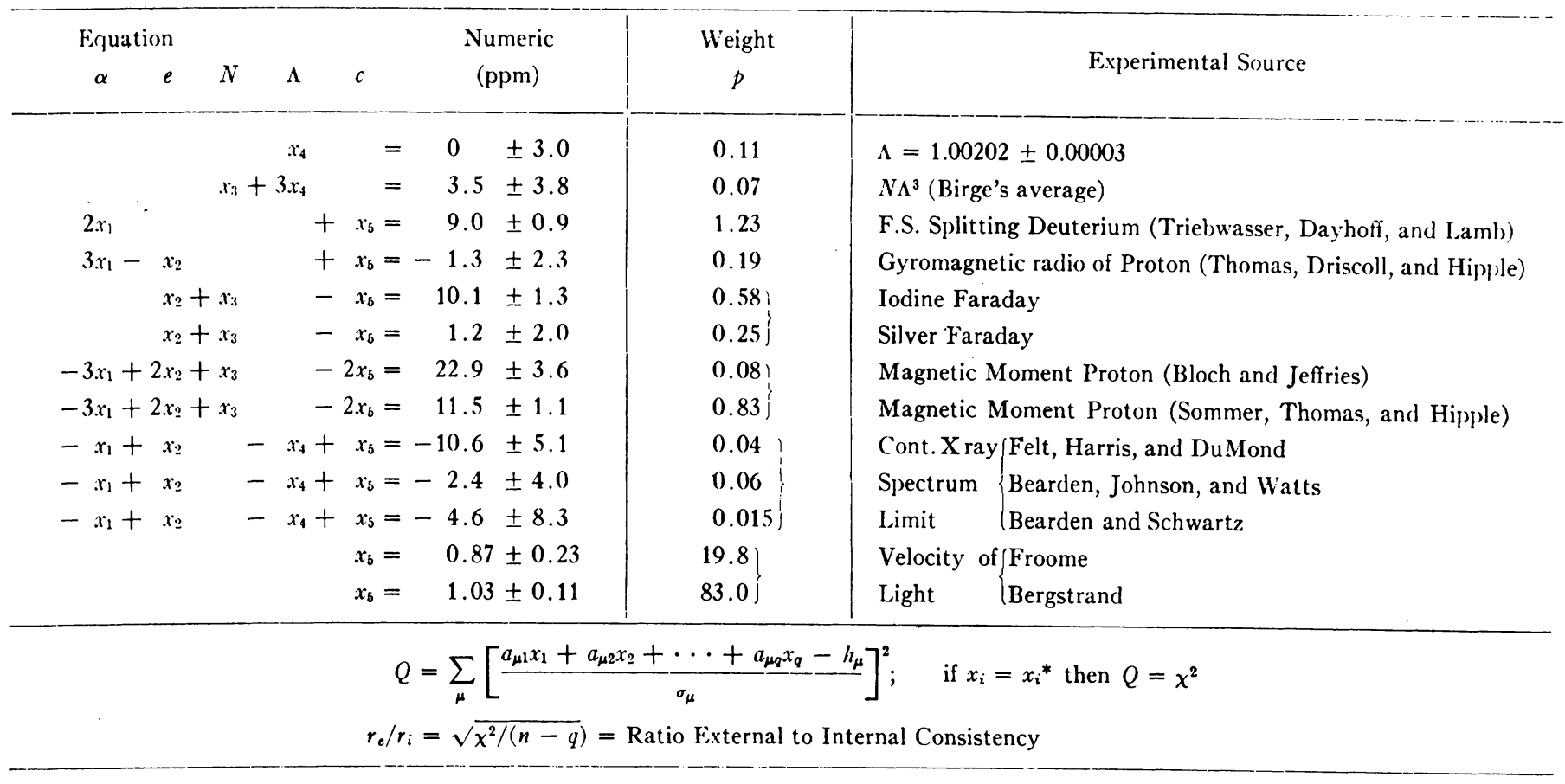

justed values from its origin value in the present instance turns out to be less than 14 parts per hundred thousand, and since the error committed by linearization through neglect of the second-order terms in the Taylor expansion is of the order of the square of this number or about 2 parts in one hundred million, it is seen to be completely negligible). Clearly (49) is the equation of the tangent plane to each surface where it passes through the "origin" point $\alpha=\alpha_{0} ; e=e_{0}$, etc.

We thus are presented with 13 equations of the form (49), one for each of the 13 observational equations in Table I, with 13 different constants $h$ and standard deviations $\pm \sigma$. These 13 equations also fall into seven different "kinds," each kind being characterized by different coefficients $(a, b, c$, etc.) of the linearized unknowns corresponding to the different powers to which $\alpha, e, N$, and $\Lambda$ are involved in the seven kinds of primitive equations. The complete linearized set is shown in Table IX.

\section{The Criterion of Least-Squares}

How may we select a set of values for the four unknowns which will do the least violence to all 13 of these more-or-less incompatible equations? Our compromise should take into account the estimated precision we attach to the different experimentally measured constants, requiring less violence to be done to the more precise data. If we assume that there are no sy'stematic errors and that the statistical distributions of the random errors of the input data (of which the $h$ 's are the mean values and the $\sigma$ 's the rms deviations) are Gaussian distributions, then both Laplace [80] and Gauss [81] have shown a simple procedure for finding the "most probable" values of the unknowns.

Consider any set of values of the unknowns, $x_{\alpha}, x_{e}$, $x_{N}$, and $x_{\Lambda}$, which may or may not be the most probable values. When we substitute these values into the left-hand members of the equations of observation we shall, in general, not obtain exactly the numbers, $h$, but there will be a slight discrepancy which we shall call the residue, $R_{\mu}$. (The subscript, $\mu$, refers to the number of the observational equation.) The residue, $R_{\mu}$, divided by the standard deviation, $\sigma_{\mu}$, of the numeric in the corresponding equation, i.e., the residue measured in units of the standard deviation, is called the "normalized residue." Now both Laplace and Gauss showed that the criterion which yielded the "most probable value" of the unknowns was to choose values for them such that when these were substituted back into the original equations the sum of the squares of the normalized residues was a minimum. In other words their criterion was to minimize the quantity, $Q$, where

$$
Q=\sum_{\mu}\left[\frac{a_{\mu 1} x_{1}+a_{\mu 2} x_{2}+\cdots+a_{\mu q} x_{\mu}-h_{\mu}}{\sigma_{\mu}}\right]^{2} .
$$


They showed that this criterion was sufficient to fix uniquely a set of values of the $x$ 's, which were the "most probable values" (under the initial assumption of Gaussian distributions), and they gave the simple well-known procedure for finding the $x$ 's which accomplished this.

Stated in its most elementary form the procedure is to multiply each of the observational equations through 1) by a weight $p_{\mu}$ taken inversely proportional to the square of the $\sigma_{\mu}$ of that equation, and 2) by the coefficient $a_{\mu}$, of $x_{1}$ as it occurs in that equation. Do this for all $n$ equations and sum the entire resulting system of equations to form a single equation to be identified as the $x_{1}$ equation. Repeat the process, this time, however, using as multipliers the coefficients, $a_{\mu 2}$, of $x_{2}$ as they occur in each equation. This total sum is to be identified as the $x_{2}$ equation. In this way form a set of $q$ equations in $q$ unknowns, each equation not only involving all $q$ of the unknowns but also being identified or labelled with one of the unknowns. These are called the "normal equations" and their solution gives those values of the $x$ 's which satisfy the criterion of least-squares. (For the elementary procedure just described to be valid, however, it is essential that the error distributions of the different equations be statistically independent, but if not, the case can be generalized in a matrix formulation which is, however, considerably more cumbersome to apply [82]-[87].)

So far we have restricted ourselves to Gaussian distributions only. However, Gauss himself was able to justify this method on a much wider base than this restriction. In 1821 he published a theory [88] which replaces the "Axiom of Maximum Likelihood" with an "Axiom of Minimum Error," or "Axiom of Maximum Weight." This permitted the extension of the principle of least-squares to include a far wider category of distribution functions than the Gaussian. In this broader theory any statistical distribution whatever is permittedrectangular-flat-topped, bimodal, or multimodal-solong as it possesses a finite second moment, i.e., a finite standard deviation. Also the distributions for the different equations of observation need not be similar. Clearly the "Axiom of Maximum Likelihood" must be abandoned as a goal if we are to deal with distributions which have no well-defined maxima (flat-topped distributions) or distributions with several maxima. Gauss showed, however, that identically the same procedure as we have described above (for finding the set of values of the unknowns whose aim is to minimize the sum of the squares of the normalized residuals), in the more general case, yields output values for the unknowns to which the smallest standard deviations and, therefore, the greatest weights can be attached. Thus, in this more general case, the goal of maximizing the weight or minimizing the standard error attachable to the output values of the $x$ 's replaces the goal of finding the most probable $x$ 's. It is strange that now, 130 years after Gauss discovered and enunciated this greatly broadened significance of least-squares, the great majority of physicists still believe the theory is applicable only to Gaussian distributions!

\section{5. $\chi^{2}$, the Measure of Compatibility of the Observational Equations}

If we substitute the least-squares adjusted values of the $x$ 's (which we shall designate as the $x^{*}$ 's) back into the observational equations and evaluate $Q$, the sum of the squares of the normalized residuals, we shall get the minimum possible value of this sum. This minimum value is called $\chi^{2}$.

$\chi^{2}=\sum_{\mu}\left[\frac{a_{\mu 1} x_{1}{ }^{*}+a_{\mu 2} x_{2}{ }^{*}+\cdots+a_{\mu q} x_{q}{ }^{*}-c_{\mu}}{\sigma_{\mu}}\right]^{2}$.

The expectation value of $\chi^{2}$ is just the number of "degrees of freedom," $n-q$, of the set of observational equations, the excess of the number of equations over the number of unknowns, and if $\chi^{2}$ greatly exceeds $n-q$ it is very likely either that systematic errors are present or else that the magnitude of the random errors of the original data have been seriously underestimated. From Fisher's $\chi^{2}$ distribution table [89] it is easy to set the upper and lower boundary values of $\chi^{2}$ corresponding to 90 per cent confidence limits. The square root of the quotient, $\chi^{2} /(n-q)$, gives $r_{e} / r_{i}$, the ratio of error by external to error by internal consistency:

$$
r_{e} / r_{i}=\sqrt{\chi^{2} /(n-q)}
$$

This quantity deserves a brief explanation. We have two criteria for fixing the scale of errors of the leastsquares adjusted output values, an a priori or internal criterion and an a posteriori or external criterion. The first of these gives an estimate of the errors to be expected in the output $x$ 's based on the estimated errors of the experimental input data, assuming absence of systematic errors. The second criterion bases its estimate of the errors in the output $x$ 's on the incompatibility of the overdetermined set of equations. There is no $a$ priori reason why these two should agree. If the incompatibility of the equations greatly exceeds the amount to be expected on the basis of the estimated random errors of the input data then the presumption of systematic error in some of the data is strong. It is a matter of considerable interest to find ways of detecting whether the rejection of some small minority of the total set of equations will render the remainder much more compatible and, if so, which ones belong to this suspect minority. We wish to be clearly understood that such a procedure can usually scarcely do more than cast suspicion of systematic error on certain data. The existence of error can only be established with certainty by discovering its physical origin as, for example, impure conditions under which the experiment was performed, failure to reduce the data correctly, or failure to make appropriate corrections for perturbing phenomena etc. But it has been proved of great value to pinpoint these suspect determinations in this way because it shows where it is worthwhile looking for trouble. 
The 13 equations of our Table I corresponding to the 13 linearized equations of Table IX were analyzed in 1952 (retaining however, $c$, the velocity of light, as one of the unknowns for adjustment), and the set was found to have a value of $\chi^{2}$.

$$
\chi^{2}=52.1
$$

Since the number of degrees of freedom, $n-q$, was only 8 , this indicates a disturbing degree of incompatibility. The 90 per cent lower and upper confidence limits on $\chi^{2}$ for this case are 2.73 and 15.51. The probability is less than one in a thousand that $\chi^{2}$ would be larger than 26.1 entirely as a result of random error. The ratio of errors by external to those by internal consistency, $r_{e} / r_{i}$, for this case equals $2.55 \pm 0.90$; in other words, one must assign errors to the adjusted output values 2.55 times larger than the errors to be expected from the estimated precision of the input data in order to make allowance for the errors indicated by the incompatibility of the equations relative to each other. Thus it was highly desirable to find, if possible, a small minority of the 13 equations whose rejection from the set would markedly improve its $\chi^{2}$ in an effort to locate the experiments under suspicion of systematic errors, which latter might then be sought for carefully.

\section{Variance Analysis of 1955-A Search for Systemati- cally Erroneous Data}

This we accomplished in 1955 by a strong-arm method [90], [91], using an electronic digital computer (Datatron). The number of equations was first trimmed ${ }^{22}$ from 13 to 11 and the number of unknowns from 5 to 4 . The machine then formed (practically exhaustively) a great number of different possible subsets (219 in fact) of these 11 equations omitting systematically, single equations, pairs of equations selected in all possible combinations, triplets similarly selected, etc. ${ }^{23}$ The machine was programmed to ring its own changes on the subsets automatically, and for each subset it performed the least-squares adjustment, determined and printed the values of the unknowns, the $\chi^{2}$, the matrix of normal equations, and the error matrix (a term which I shall explain a little later). In this way we pinned suspicion of systematic error on four experiments, the determination of the proton magnetic moment by Bloch and Jeffries, the Faraday determination using the silver coulometer, and two out of the three determinations of the short wavelength limit of the continuous $\mathrm{X}$-ray

22 This labor-saving device was as follows. Two equations were eliminated by treating $c$ as an exactly known constant rather than as an unknown to be determined by least-squares adjustment. This was amply justified by the precision with which $c$ was even then known and by the very weak correlation between this unknown and the other unknowns that had been revealed in the 1953 adjustment. The two eliminated equations corresponded to the respective measurements of $c$ by Froome and by Bergstrand which were in excellent agreement.

${ }^{23}$ To minimize the labor, 1) not more than one equation of any one "kind" was included in any one subset, and 2) with this restriction all subsets were examined which overdetermined all the unknowns. To just this extent the examination of subsets fell short of being completely exhaustive. spectrum. ${ }^{24}$ Our final censored set of equations, with the four suspects eliminated, had 3 degrees of freedom and yielded the remarkably good $\chi^{2}$ value of 3.25 as compared to the previous 52.1. The value of $r_{e} / r_{i}$ was 1.041. The 90 per cent confidence interval for $r_{e} / r_{i}$ with 3 degrees of freedom is $0.594<r_{e} / r_{i}<2.795$.

\section{Cohen's Formulas for the Standard Errors of the Residues and for the "Indirect Values" and their Standard Errors}

Cohen has derived very simple and useful formulas for computing the standard errors of the residues of a least-squares adjustment. These also lead to simple expressions for computing what I shall call "the indirect value" implicit in a least-squares adjustment. Consider any one of the directly-measured input quantities, $c_{\mu}$, of a least-squares adjustment. It turns out that the least-squares adjusted output value of this same quantity can be regarded as compounded of two contributions; one is the direct input value, $c_{\mu}$, while the other is an effective or "indirect value" which is determined by the combined action implicit in all of the other data. This latter "indirect" value is the value of $c_{\mu}$ (call it $\left.c_{\mu}{ }^{i}\right)$ which would be deduced from a least-squares analysis from which the directly observed datum had been omitted.

Specifically Cohen has shown [92], [93] that the "variance" (square of the rms deviation, $\sigma$ ) of the cifference, $c_{\mu}-c_{\mu}{ }^{*}$, between the directly-measured input value, $c_{\mu}$, and the least-squares adjusted output value, $c_{\mu}{ }^{*}$, of this same quantity is given by $\sigma_{\mu}{ }^{2}-\sigma_{\mu}{ }^{* 2}$, where $\sigma_{\mu}^{2}$ is the variance of the input datum, and $\sigma_{\mu}{ }^{* 2}$ is the variance of its least-squares adjusted value.

In this same terminology the indirect value, $c_{\mu}{ }^{i}$, and its variance $\sigma_{\mu}{ }^{i 2}$ are given by

$$
\begin{aligned}
& c_{\mu}{ }^{i}=\frac{{\sigma_{\mu}}^{2} c_{\mu}{ }^{*}-{\sigma_{\mu}}^{* 2} c_{\mu}}{{\sigma_{\mu}}^{2}-\sigma_{\mu}{ }^{* 2}}=c_{\mu}{ }^{*}+\frac{\sigma_{\mu}{ }^{* 2}}{{\sigma_{\mu}}^{2}-\sigma_{\mu}{ }^{* 2}}\left(c_{\mu}{ }^{*}-c_{\mu}\right) \\
& \sigma_{\mu}^{i 2}=\frac{\sigma_{\mu}^{2} \sigma_{\mu}{ }^{* 2}}{{\sigma_{\mu}}^{2}-\sigma_{\mu}{ }^{* 2}} .
\end{aligned}
$$

This expression for the variance implies that the statistical weights, $p$ (proportional to the reciprocals of the variances), are related by

$$
p_{\mu}{ }^{*}=p_{\mu}{ }^{i}+p_{\mu}, \quad p=C / \sigma^{2} .
$$

If we use weights rather than variances, (53) takes on the simple form

$$
c_{\mu}{ }^{*}=\frac{p_{\mu} c_{\mu}+p_{\mu}{ }^{i} c_{\mu}{ }^{i}}{p_{\mu}+p_{\mu}{ }^{i}}
$$

which is merely the statement that the least-squares adjusted value is the weighted mean of the direct input value and the indirect value.

24 The one determination which was retained, that of Bearden and Schwarz [48], suffers from less suspicion of incompatibility with the majority of the equations, more because of its low weight than because of its close agreement with them. It also could have been omitted with little effect on the output values. 
TABLE X

Direct and Indirect Evaluation of Fundamental Constants (1952 Adjustment)

\begin{tabular}{|c|c|c|c|c|}
\hline Constant & $\begin{array}{c}\text { Direct Value } \\
\text { (Experimental) }\end{array}$ & Indirect Value & Least-Squares Solution & $\begin{array}{l}\text { Residue of Least- } \\
\text { Squares Solution } \\
\text { (ppm) }\end{array}$ \\
\hline $\begin{array}{l}\Lambda \\
N \Lambda^{3} \\
\gamma\end{array}$ & $\begin{array}{l}1.002020 \pm 0.000030 \\
6,061.79 \pm 0.23 \cdot 10^{20} \\
(26,752.3 \pm 0.6) \mathrm{emu}^{-1} \mathrm{~s}^{-1}\end{array}$ & $\begin{array}{l}1.002073 \pm 0.000015 \\
6,062.90 \pm 0.39 \\
26,751.9 \pm 0.4\end{array}$ & $\begin{array}{l}1.002063 \pm 0.000013 \\
6,062.08 \pm 0.20 \\
26,752.0 \pm 0.5\end{array}$ & $\begin{array}{r}43 \pm 27 \\
48 \pm 19 \\
-11 \pm 20\end{array}$ \\
\hline$F\left\{\begin{array}{l}(\mathrm{I}) \\
(\mathrm{Ag})\end{array}\right.$ & $\begin{array}{l}(9,652.15 \pm 0.13) \mathrm{emu} / \mathrm{mole} \\
(9,651.29 \pm 0.19) \mathrm{emu} / \mathrm{mole}\end{array}$ & $\begin{array}{l}9,651.90 \pm 0.15 \\
9,652.21 \pm 0.11\end{array}$ & $\begin{array}{l}9,652.01 \pm 0.10 \\
9,652.01 \pm 0.10\end{array}$ & $\begin{array}{l}-11 \pm 9 \\
78 \pm 17\end{array}$ \\
\hline $\begin{array}{l}\mu^{\prime}\left\{(\mathrm{BJ})^{1}\right. \\
(\mathrm{HST})^{2}\end{array}$ & $\begin{array}{l}2.79237 \pm 0.00010 \\
2.79268 \pm 0.00003\end{array}$ & $\begin{array}{l}2.79270 \pm 0.00005 \\
2.79263 \pm 0.00005\end{array}$ & $\begin{array}{l}2.79267 \pm 0.00003 \\
2.79267 \pm 0.00003\end{array}$ & $\begin{array}{l}109 \pm 34 \\
-5 \pm 6\end{array}$ \\
\hline$\left((F H D)^{3}\right.$ & $12,370.02 \pm 0.63) \mathrm{emu} \mathrm{cm}$ & $12,372.40 \pm 0.17$ & $12,372.23 \pm 0.16$ & $179 \pm 48$ \\
\hline ? $3(\mathrm{BJW})^{4}$ & $(12,371.03 \pm 0.48) \mathrm{emu} \mathrm{cm}$ & $12,372.37 \pm 0.17$ & $12,372.23 \pm 0.16$ & $97 \pm 39$ \\
\hline$\underset{x}{x}\left((\mathrm{BS})^{5}\right.$ & $(12,370.77 \pm 1.03) \mathrm{emu} \mathrm{cm}$ & $12,372.28 \pm 0.17$ & $12,372.23 \pm 0.16$ & $119 \pm 81$ \\
\hline
\end{tabular}

1 Bloch and Jeffries.

${ }^{2}$ Hipple, Summer, and Thomas.

${ }^{3}$ Felt, Harris, and DuMond.

${ }^{4}$ Bearden, Johnson, and Watts.

${ }^{5}$ Bearden and Schwarz.

${ }^{6} \mathrm{X}$-ray SWL $=$ Short wavelength limit of the continuous X-ray spectrum.

18. Application of Cohen's Formulas to Detection of Systematic Error

These simple formulas of Cohen's are of considerable value in any critical examination of the residues of a least-squares adjustment. In fact a comparison of the directly measured input value of each constant with 1) the indirect value of the same constant, 2) its leastsquares adjusted value and 3) the residue of the leastsquares solution expressed with its rms error [(53) above] affords a far easier way (almost but not quite as searching) than the "strong-arm" $\chi^{2}$ exploration described above for locating data suspect as to systematic error.

In Table $\mathrm{X}$ we show the direct and indirect values, the least-squares solution, and the residues, each with their rms deviations for ten of the input data of our 1952 least-squares adjustment. ${ }^{25}$ We see that the iodine coulometer value of the Faraday [4], [5], for example, is much more consistent with the indirect value than is the silver voltameter value [3]. The difference between the iodine value and the indirect value is $53 \pm 26 \mathrm{ppm}$ while the silver Faraday differs from the indirect value by $142 \pm 30 \mathrm{ppm}$. The existence of possible sources of experimental systematic error in the silver measurement from possible electrolytic changes in isotopic constitution and other effects is discussed in Part I, and this physical evidence adds weight to the statistical implications.

Similarly the indirect value of the proton magnetic moment agrees with Sommer, Thomas, and Hipple's value [32] while the original Bloch and Jeffries inverse

${ }_{25}$ The measurements of $\Delta E_{D}$, the fine structure splitting in deuterium, and, $c$, the velocity of light, have been omitted from this table because the direct measurements of these items have so much more weight than the indirect values that the limitations of numerical accuracy in the calculation preclude a meaningful evaluation of said indirect values and their error measures. cyclotron result [33], [34] does not. The systematic error in the latter work, as we have pointed out in Part I, was later confirmed by Trigger's [36] re-analysis of the experiment and by the modified experiment of Collington, Dellis, Sanders, and Turberfield [35] at Oxford.

The short wavelength limit data [46]-[49] are also seen to be all more-or-less in significant disagreement with the indirect data, and plausible experimental reasons for this are adduced in Part I.

A word of caution about this simplified approach for finding systematically erroneous data is in order at this point. Of the 13 items which were included in this preliminary analysis we have deduced reasons to reject five. This represents a rather drastic censoring and should be carried out with care. As soon as we eliminate one item the values of all of the other adjusted output values change. Hence strictly we should re-evaluate the leastsquares solution after each rejection in order to determine a new basis for the subsequent rejections. Furthermore the decision to reject a datum as discrepant might conceivably depend on the order in which other data were rejected prior to it. It is quite possible, in general, that a particular experimental result is in disagreement with the indirectly determined value, not because of an error in the experiment itself, but because some other error-ridden experiment is strongly distorting the indirect value. If the latter experiment were rejected first, the former one might well be retained. This is particularly liable to occur if the total degrees of freedom of the system is small so that each experimental item can contribute significantly to the over-all result. These qualifications explain why I have referred to this method as "almost but not quite as searching" as that of the exhaustive exploration of many subsets for their $\chi^{2}$ values. 
19. Criteria Used in the Selection and Rejection of Data for Least-Squares Adjustment

One must be especially careful not to apply the method of least-squares blindly; it is not a substitute for careful selection of data. I have just spoken of ways of detecting the probable existence and location of systematically erroneous error contributors in a leastsquares adjustment. If a sufficiently strong case for systematic error in a given datum can be made on other grounds than mere incompatibility with the majority of equations in a least-square set, i.e., on physical or cogently logical grounds, then I believe rejection of such a datum is warranted. Incompatibility with a large majority of other consistent data, if it be sufficiently glaring, of course may also be defended as warranting rejection, but I believe in being extremely cautious in rejecting outlying data solely because they are outlying. Fisher has said, "If you must reject data, reject those which lie closest to the mean because they have the least effect on the latter!"

In a least-squares adjustment any input observation with sufficiently large variance (low accuracy) carries little weight (in inverse proportion to its variance) and hence may be omitted without seriously affecting the results. There is, however, a more important reason for omitting data of low weight. When an experimenter designs his experiment he must carefully consider the possible presence of systematic error as well as the presence of random error. The random error of the final result can be reduced by duplication and repetition since these errors are different in each repetition; the systematic errors on the other hand do not cancel out but remain. Now, it is proper in an experiment to reduce any possible source of systematic error to a point where it may be of the order of, say, one-tenth of the random error of observation. In this way the systematic error will be of the same order as, or smaller than, the random error of the final quoted result. However, it is neither feasible nor practical to do much better than this in the suppression of systematic error.

Thus an experiment with a quoted error which is large compared to another similar but more precise measurement may well be affected with a systematic error which is large compared to the accuracy of the second experiment. This systematic error would then, a fortiori, be large compared to the accuracy which might be claimed for the weighted mean of the two results. It, therefore, would be inappropriate to include the less precise observation in a weighted mean.

Grounds for suspicion of systematic error need not arise from experimental causes alone. Theoretical sources of errors, mistakes in calculation, and other oversights, are not uncommon. Also a theoretical uncertainty may exist as in the case of the remarkably accurate measurement of $\Delta \nu_{H}$, the hyperfine structure splitting in hydrogen by Prodell and Kusch [94], accurate to $0.2 \mathrm{ppm}$. (For a discussion see [95]-[97].) The trouble in this case in our 1952 and 1955 adjustments was that the precision of the measurement was higher than the existing state of the theory of the experiment could handle. In particular, a correction factor in the theoretical expression connecting $\Delta \nu_{H}$ with $\alpha, c$, and $R_{\infty}$, a term required to correct for the finite extension of the proton's magnetic dipole moment, could not be evaluated with sufficient accuracy in the light of existing theoretical knowledge of the structure of the proton at that time. (An empirical evaluation of this correction factor may soon be available however, thanks to the work of Hofstadter at Stanford [98]-[104].)

In accord with the above-mentioned criteria we have rejected in the present adjustments almost all of the historically important early experiments, including many which were considered important as late as 1947 . Physicists brought up on the textbook accounts of how such atomic constants as $e, h$, and $m$, were measured for the first time in famous historically important experiments such as R. A. Millikan's oil-drop experiment or his studies of the photoelectric effect, Perrin's Brownian motion determinations of the Avogadro number, or J. J. Thomson's deflection experiments on cathode rays to determine $e / m$, may perhaps be surprised to see how little remains of these traditional methods in the present high-precision evaluation. After correction for their known systematic errors, and within their estimated precision ranges, such early measurements are not inconsistent with the later, more precise results, but they are relatively so much less accurate as to carry little weight and hence exert but small influence in the present adjustment, an influence which furthermore is likely to be biased by systematic errors as we have just explained. This rejection is not only wise, therefore, but it also saves a great deal of computational labor.

\section{The Least-Squares Adjustment of 1955}

In the latest adjustment made by Cohen and myself, that of 1955 , we have, as already mentioned, assumed the velocity of light to be an exactly known constant

$$
c=299793.0 \mathrm{~km} \mathrm{sec}^{-1},
$$

and we have rejected the four discrepant experimental data, silver Faraday, Bloch and Jeffries inverse cyclotron proton magnetic moment, and the two X-ray short wavelength limit measurements. The origin values and the four linearized dimensionless variables used in this adjustment are shown in Table XI.

With these definitions the linearized observational equations then assume the form shown on Table XII. The experiment which yielded each equation is given in the last column.

Note that we have used as the first input datum the 1947 Bragg "Edict" value, ${ }^{15} \Lambda=1.00202 \pm 0.0003$ for the $\mathrm{X}$-unit-milliangstrom conversion factor. (See discussion of this "edict," and its bases in Part I, Section 3.) This is because our discovery of the insecure bases upon which this value rests (Tyrén's determination with no correc- 
TABLE XI

The system of seven equations in four unknowns which is obtained when we exclude those data which appear to be discordant with the remainder of our data is given below. As has been mentioned above we assume the velocity of light to be an exact constant in this analysis, its value being

$$
c=299793.0 \mathrm{~km} \mathrm{sec}^{-1} \text {. }
$$

(The actual standard deviation to be assigned to this number is $\pm 0.3 \mathrm{~km} \mathrm{sec}-1$, but this error has a negligible effect on the numerical results of the least-squares analysis.)

The origin values and the linearized variables are taken to be:

$$
\begin{array}{ll}
x_{1}=\frac{\alpha-\alpha_{0}}{\alpha_{0}} \cdot 10^{5} & \alpha_{0}=0.007297000, \\
x_{2}=\frac{e-e_{0}}{e_{0}} \cdot 10^{5} & e_{0}=4.802200 \cdot 10^{-10} \mathrm{esu}, \\
x_{3}=\frac{N-N_{0}}{N_{0}} \cdot 10^{5} & N_{0}=0.6025000 \cdot 10^{24} \mathrm{~mole}^{-1}, \\
x_{4}=\frac{\Lambda-\Lambda_{0}}{\Lambda_{0}} \cdot 10^{5} & \Lambda_{0}=1.0020200 .
\end{array}
$$

predict that the effect on the entire adjustment, therefore, cannot be significantly large.

The four normal equations in the four unknowns are formed from the observational equations by the prescription already described. These appear at the bottom of Table XII, and you note that each normal equation, beside involving all four unknowns, is labelled with one of the unknowns, a different one for each normal equation. You also note that the coefficients of the normal equations form a matrix symmetric about its major diagonal in which each element is labelled with a pair of the unknowns. The solution of this set of normal equations for the $x_{i}$ 's gives the least-squares adjusted values of these variables. But this is not all; the inverse of the matrix formed by the coefficients of the normal equations is the error (or variance) matrix, also symmetric, and containing all the important information needed to compute the error measures of the output values.

TABLE XII

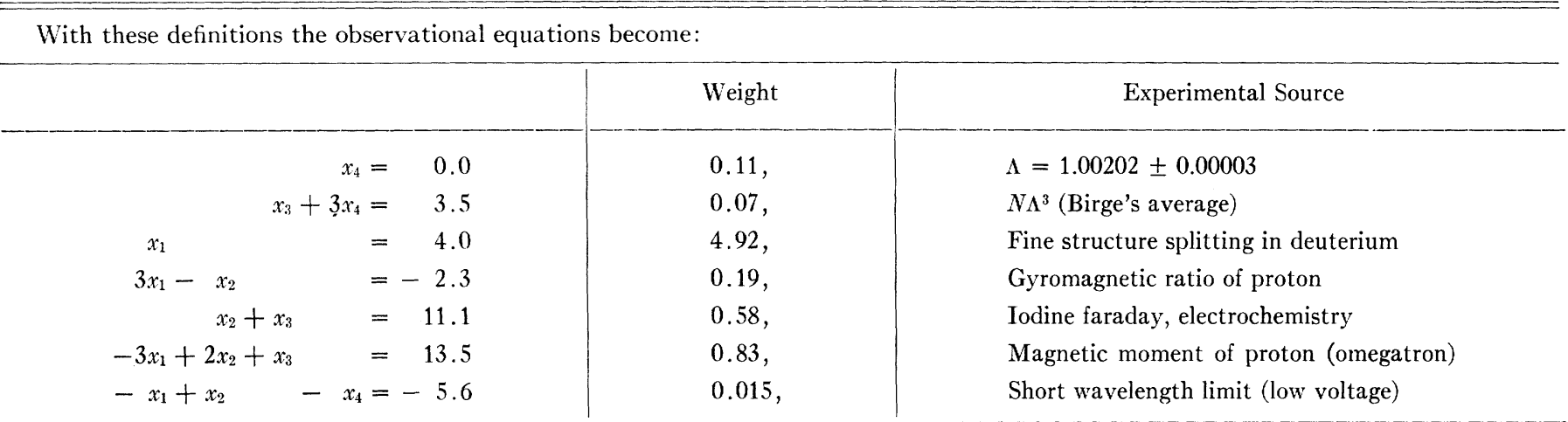

The normal equations are formed according to the usual rules; they are

$$
\left\{\begin{array}{lrr}
(\alpha) & 14.115 x_{1}-5.565 x_{2}-2.490 x_{3}+0.015 x_{4}= & -15.162 \\
(e) & -5.565 x_{1}+4.105 x_{2}+2.240 x_{3}-0.015 x_{4}= & 29.201 \\
(N) & -2.490 x_{1}+2.240 x_{2}+1.480 x_{3}+0.210 x_{4}= & -17.888 \\
(\Lambda) & 0.015 x_{1}-0.015 x_{2}+0.210 x_{3}+0.755 x_{4}= & 0.819 .
\end{array}\right.
$$

tion for Lamb shift) came subsequent to our making this adjustment.

I should also point out that three of these equations, the third, fourth, and sixth, have numerical constants suffering from a slight systematic bias of about $14.2 \mathrm{ppm}$. This results from a computational error committed by Karplus and Kroll [43] in the derivation of the fourth-order quantum-electrodynamic correction term in the theoretical formula for $\mu_{e} / \mu_{0}$, the anomalous ratio of the electron magnetic moment to the Bohr magneton, an error recently discovered and pointed out by Petermann [72] [see Section 11(a)] and verified by Karplus and by Sommerfield. Because of this, the numeric 4.0 should be changed to 2.6 , the numeric -2.3 should be changed to -3.7 , and the numeric 13.5 should be changed to 14.9 . The changes correspond respectively to only about $3.2,0.6$, and 0.4 of a standard deviation of the respective input quantities, and we can
Table XIII shows the normal equations and the inverse matrix or error matrix whose diagonal elements are proportional to the variances of the four output values and whose off-diagonal elements are proportional to the covariances connecting each possible pair of different output values. Each element, $d_{i j}$, of this inverse matrix is given by the minor of the corresponding element, $b_{i j}$, of the determinant of the normal equation matrix divided by the determinant itself. The single constant of proportionality relating this inverse matrix to the covariance matrix may be chosen either so that the variances and covariances reflect 1) the a priori estimated errors (those based on "internal" consistency, i.e., the errors assigned to the input data), or 2) the a posteriori estimated errors (those based on "external" consistency, i.e., on the degree of mutual incompatibility of the observational equations). In the first case one merely multiplies all the elements of the 
TABLE XIII

The normal equations are formed according to the usual rules; they are

$$
\begin{aligned}
& \text { ( } \alpha) \quad 14.115 x_{1}-5.565 x_{2}-2.490 x_{3}+0.015 x_{4}=-15.162 \\
& \text { (e) }-5.565 x_{1}+4.105 x_{2}+2.240 x_{3}-0.015 x_{4}=29.201 \\
& (N)-2.490 x_{1}+2.240 x_{2}+1.480 x_{3}+0.210 x_{4}=17.888 \\
& \text { (A) } \quad 0.015 x_{1}-0.015 x_{2}+0.210 x_{3}+0.755 x_{4}=0.819 \text {. }
\end{aligned}
$$

It should be noted here that the normal equations are to be formed directly from the coefficients of the quadratic form, $Q$, and that no simplification or cancellation should be made in the equations. This is because the coefficients of the normal equations have a significance which is not limited to the determination of the values of the $x_{i}$. An alteration in the equations which would not alter the solution (such as the cancellation of a common factor in one of the equations, or even the reordering of the equations in the set) can destroy the identifications of the coefficients of the normal equations as the weight matrix of the solution. is

The error (or variance) matrix of the solution is the inverse of the matrix of the coefficients of the normal equations. Hence the error matrix

$$
V=\left(\begin{array}{rrrr|l}
0.1989 & -0.5760 & 0.5603 & 0.1633 \\
0.5760 & 3.4478 & -4.4319 & 1.2898 \\
-0.5603 & -4.4319 & 6.7167 & -1.9452 \\
0.1633 & 1.2898 & -1.9452 & 1.8879
\end{array}\right) \text { }
$$

\section{TABLE XIV}

The solution for the variables is

$$
\left\{\begin{array}{l}
x_{1}=3.92 \\
x_{2}=13.72 \\
x_{3}=-2.37 \\
x_{4}=1.94 .
\end{array}\right.
$$

The value of $\chi^{2}$ for this solution is

$$
\chi^{2}=3.25
$$

This is to be compared to the value 52.1 which was obtained in the preliminary adjustment. The major change in the variables caused by the deletion of those data suspected of systematic error is an increase in $x_{3}$ of 2.23 and an attendant decrease in $x_{4}$ of 2.35 ; this corresponds to a change in Avogadro's number $N$ of $22.3 \mathrm{ppm}$ and a change in the conversion factor $\Lambda$ of $-23.5 \mathrm{ppm}$.

The value of $\chi^{2}$ can be expressed equivalently in terms of the ratio of external to internal consistency.

$$
r_{e} / r_{i}=\sqrt{\chi^{2} /(n-q)}=1.041 \text {. }
$$

inverse matrix by the single constant, $C$, in the relation which was chosen between the weights and the reciprocals of the variances of the input data, i.e., the relation

$$
p_{\mu}=C / \sigma_{\mu}{ }^{2} \text {. }
$$

In the second case we expand this scale of variance magnitudes by the multiplying factor

$$
\chi^{2} /(n-q)
$$

In the present case the two criteria coincide in giving essentially the same output errors.

Table XIV shows the least-squares adjusted values of the $x$ 's obtained by solution of the normal equations and also the value of $\chi^{2}$ obtained by resubstituting these adjusted values into the observational equations and summing the squares of the normalized residues. As can be seen, the ratio of errors by external to those by internal consistency is very close to unity in the present case.

Table XV shows the covariance matrix of the 1955 adjustment which, like the normal and inverse matrices, has, as you see, elements symmetric about the major
TABLE XV

Covariance Matrix (1955 Adjustment)

\begin{tabular}{l}
\hline Elements of the matrix are in units of (relative parts per million $)^{2}$ \\
\hline \\
\hline
\end{tabular}

TABLE XVI

Correlation Coefficients (1955 Adjustment)

\begin{tabular}{l|r|r|r|r|r|r|r}
\hline & \multicolumn{1}{c|}{$e$} & \multicolumn{1}{c|}{$m$} & \multicolumn{1}{c|}{$h$} & \multicolumn{1}{c|}{$\alpha$} & \multicolumn{1}{c|}{$\Lambda$} & \multicolumn{1}{c|}{$N$} & \multicolumn{1}{c}{$F$} \\
\hline$e$ & 1.00 & 0.95 & 0.99 & 0.70 & 0.51 & -0.92 & -0.47 \\
$m$ & 0.95 & 1.00 & 0.97 & 0.43 & 0.52 & -0.94 & -0.60 \\
$h$ & 0.99 & 0.97 & 1.00 & 0.63 & 0.52 & -0.94 & -0.51 \\
$\alpha$ & 0.70 & 0.43 & 0.63 & 1.00 & 0.27 & -0.48 & 0.03 \\
$\Lambda$ & 0.51 & 0.52 & 0.52 & 0.27 & 1.00 & -0.55 & -0.42 \\
$N$ & -0.92 & -0.94 & -0.94 & -0.48 & -0.55 & 1.00 & 0.77 \\
$F$ & -0.47 & -0.60 & -0.51 & 0.03 & -0.42 & 0.77 & 1.00 \\
\hline
\end{tabular}

diagonal. The variances of each of the quantities, $e, m, h$, etc., are the numbers on the major diagonal, and they are estimates of the $\sigma^{2}$ 's, i.e., the mean-square deviations from the mean, of the error distributions attributed to each of these output quantities. The off-diagonal terms, called the covariances, give the mean values of the product of the random errors in each pair of quantities. Thus, if $\epsilon_{e}$ and $\epsilon_{\alpha}$ are individual errors selected at random from the joint error distribution of these two variables, the mean product, $\left\langle\epsilon_{e} \epsilon_{\alpha}\right\rangle$, averaged over the joint distribution is seen from this table to be $62\left(\mathrm{ppm}^{2}\right)$. That this mean value does not vanish results from the fact (which generally is true of the output values of any least-squares adjustment) that these output values are not statistically completely independent quantities; on the contrary, they are statistically correlated, that is to say, the free selection at random of a value from the statistical error distribution of one of the quantities biases 
our freedom of selection of values from the error distributions of the others.

If we divide any covariance, $v_{i j}$, in the table by the standard deviations, $\sigma_{i}$ and $\sigma_{j}$, of its two variables, the quotient gives the correlation coefficient, $r_{i j}$, connecting those two variables. $r_{i j}$ can assume only values between -1 and +1 . These concepts of statistical correlation, since they are somewhat unfamiliar to many physicists, call for a few words of explanation. (See Table XVI.)

\section{Correlation Coefficients, Covariances, and the General- ized Formula of Error Propagation}

Each quantity subject to random or accidental error (frequently called a "random variable") may conveniently be thought of as a sample taken at random from a "universe" of values which group themselves around a mean value according to some frequency law. For each such random variable, $x_{i}$, one is to think then of the implied universe of values from which it is selected. This universe may be described by giving some of its parameters. Thus, if the universe is known to be Gaussian, for example, then prescription of its first and second moments, i.e., its mean value, $\mu_{i}$, and its variance, $\sigma_{i}{ }^{2}$, are sufficient.

Two such random quantities are observationally independent if the random selection of a sample value from one universe in no wise affects or biases the free selection of a sample from the other universe. If, for example, the two variables are connected by a strict functional relationship, so that the value of either one is uniquely determined by that of the other, the variables are completely correlated and the correlation coefficient has absolute value unity. (It may be +1 or -1 depending on whether an increase in one quantity corresponds to an increase or a decrease in the other.) On the other hand, if one of the two random variables is a function of the other and also of still other completely independent random variables, then these two will be partially but not completely correlated, and the correlation coefficient connecting them will have a value somewhere between -1 and +1 . In general, if we have a set of statistically independent random variables, $y_{\mu}$, then a second set of variables, $x_{i}$, obtained by linear transformation on the $y_{\mu}$

$$
x_{i}=\sum_{\mu} \lambda_{i \mu} y_{\mu}
$$

will not be statistically independent since a given one of the variables, $y_{\mu}$, and hence a given source of error, is present in more than one of the variables, $x_{i}$. This situation exists in a least-squares adjustment.

It follows from these facts that use must be made of all of the elements of the error matrix of a least-squares adjustment if we are to compute the error estimates (standard deviations) of functions depending on the output values of that least-squares adjustment. It is not enough simply to combine the squares of the standard deviations of those correlated output values upon which the function depends, as one is accustomed to do when the variables are statistically independent. We must also take into account cross-product terms whose importance and algebraic signs depend on the correlation coefficients between pairs of quantities. To each output value, $x_{i}$, of the least-squares adjustment there corresponds a standard deviation, $\sigma_{i}$, and a variance $v_{i i}=\sigma_{i}{ }^{2}$, and to each pair of output values $\left(x_{i}, x_{j}\right)$ there corresponds a covariance $v_{i j}=r_{i j} \sigma_{i} \sigma_{j}$, the entire set of these forming the covariance matrix. In general all these quantities are needed to compute propagated errors.

To compute the standard error, $\sigma_{f}$, in a function, $f\left(x_{1}, x_{2}, x_{3}\right)$, of say three of the variables, we must use the generalized formula of error propagation:

$$
\begin{aligned}
\sigma_{f}{ }^{2}= & \alpha_{1}^{2} \sigma_{1}{ }^{2}+\alpha_{2}^{2} \sigma_{2}{ }^{2}+\alpha_{3}^{2} \sigma_{3}{ }^{2} \\
& +2 r_{12} \alpha_{1} \alpha_{2} \sigma_{1} \sigma_{2}+2 r_{13} \alpha_{1} \alpha_{3} \sigma_{1} \sigma_{3}+2 r_{23} \alpha_{2} \alpha_{3} \sigma_{2} \sigma_{3} .
\end{aligned}
$$

In this formula the $\alpha_{i}$ 's are the partial derivatives, $\partial f / \partial x_{i}$, of the function, $f$. The first three terms will be recognized as the familiar terms of error propagation for statistically independent variables. The last three, the cross-product terms, are the new ones required by the existence of correlation between the $x$ 's, and they may either increase or diminish $\sigma_{f}{ }^{2}$ since both the $\alpha_{i}$ 's and the $r_{i j}$ 's may be either positive or negative. This formula may also be more simply written in terms of the covariances $v_{i j}=r_{i j} \sigma_{i} \sigma_{j}$ as

$$
\begin{aligned}
\sigma_{f}{ }^{2}= & \alpha_{1}{ }^{2} \sigma_{1}{ }^{2}+\alpha_{2}{ }^{2} \sigma_{2}{ }^{2}+\alpha_{3}{ }^{2} \sigma_{3}{ }^{2} \\
& +2 v_{12} \alpha_{1} \alpha_{2}+2 v_{13} \alpha_{1} \alpha_{3}+2 v_{23} \alpha_{2} \alpha_{3},
\end{aligned}
$$

or, in general, simply as

$$
\sigma_{f}^{2}=\sum_{i} \sum_{j} v_{i j} \sigma_{i} \sigma_{j}
$$

A simple, in fact almost trivial, example will serve to illustrate the need for this generalized formula of errors. Consider an imprecisely known quantity, $x$, with standard deviation $\pm \sigma_{x}$. What is the standard deviation of the following simple function of $x$, namely $y=x+x=2 x$ ? The familiar square-root-of-sum-of-squares rule for independent variables, if applied to $x+x$ as though the two $x$ 's were statistically independent, would give us $\sigma_{y}=\sqrt{\sigma_{x}^{2}+\sigma_{x}^{2}}=\sqrt{2} \sigma_{x}$. But clearly the standard deviation of $2 x$ is $2 \sigma_{x}$, not $\sqrt{2} \sigma_{x}$. This paradox is resolved when we apply the above generalized formula remembering that the correlation coefficient between the two identical $x$ 's is clearly unity. The generalized formula with its required cross-product term gives

$$
\sigma_{y}=\sqrt{\sigma_{x}^{2}+2 \sigma_{x} \sigma_{x}+\sigma_{x}^{2}}=2 \sigma_{x}
$$

which clearly yields the correct result.

From our chosen origin values and the least-squares adjusted values of the four $x$ 's, which you recall were the relative deviations in parts per hundred thousand from the origin values, we now readily compute the values of the primitive unknowns. These results are shown in Table XVII. 


\section{TABLE XVII}

Origin Values, Linearized Unknowns and Least-Squares Adjusted Primitive Variables of 1955 Adjustment

\begin{tabular}{c|l|c}
\hline \hline Origin Values & Linearized Unknowns & Least-Squares Adjusted Values of $x^{\prime}$ 's \\
\hline$\alpha_{0}=0.007297000$ & $x_{1}=\left[\left(\alpha-\alpha_{0}\right) / \alpha_{0}\right] \cdot 10^{5}$ & $x_{1}^{*}=3.92$ \\
$e_{0}=4.802200 \cdot 10^{-10} \mathrm{esu}$ & $x_{2}=\left[\left(e-e_{0}\right) / e_{0}\right] \cdot 10^{5}$ & $x_{2}^{*}=13.72$ \\
$N_{0}=0.6025000 \cdot 10^{24} \mathrm{~mole}^{-1}$ & $x_{3}=\left[\left(N-N_{0}\right) / N_{0}\right] \cdot 10^{5}$ & $x_{3}^{*}=2.37$ \\
$\Lambda_{0}=1.0020200$ & $x_{4}=\left[\left(\Lambda-\Lambda_{0}\right) / \Lambda_{0}\right] \cdot 10^{5}$ & $x_{4}{ }^{*}=1.94$ \\
\hline
\end{tabular}

Resulting Least-Squares Adjusted Values of the Primitive Variables

$\alpha^{*}=(7.29729 \pm 0.00003) \cdot 10^{-3}$

$N^{*}=(6.02486 \pm 0.00016) \cdot 10^{23} \mathrm{~mole}^{-1}$ $e^{*}=(4.80286 \pm 0.00009) \cdot 10^{-10} \mathrm{esu}$

$\Lambda^{*}=1.002039 \pm 0.000014$

\section{Results of the 1955 Adjustment and Comparison with Earlier Adjustments}

By combining these four adjusted values with the list of auxiliary constants, such as $R_{\infty}, c$, certain simple atomic masses, etc. (the list, in fact, given in Table I), it becomes a simple matter to compute an impressive array of useful constants and conversion factors of physics and chemistry. Such a list of derived values, even though incomplete, easily fills five pages.

On examining these tables some readers of this paper, accustomed to the mks system of units, may be disappointed to discover that our table of output values is not expressed in these units, but in the cgs system. All the disciplines of physics and chemistry meet and are represented in the study of the fundamental constants, and the truth is that the mks system has not been adopted generally in all these domains. The Rydberg constant in reciprocal meters or the rest-mass of the electron in kilograms would seem strange to say the least. One hesitates to express the normal mole volume of an ideal gas in cubic meters per kilogram molecule. Kilogram-molecule units for the gas constant, the Avogadro number, or the Faraday constant, would seem equally unfamiliar and likely to be misunderstood. The mks system is primarily an engineering system whose primary utility lies in the field of electrical engineering and classical electromagnetic theory. We have retained the cgs units because of their wide use in all fields of physics and chemistry. It is a very simple matter of course to convert from the cgs to the mks system. Perhaps in our next adjustment Cohen and I may give such of the constants as are of particular interest to those who prefer mks units in terms of both systems so everyone will be happy. At present we are concerned with much more vital questions.

The great majority of the constants and conversion factors which we have computed and tabulated for your use have error measures (relative standard deviations) of the order of a few parts per hundred thousand. As far as our overdetermined set of input data, the best available in 1955, can tell us, these are the "best" values of the constants and the uncertainties with which we knew them as of that year. At any one epoch of time this is the best that can be done. But experience teaches us, when new experimental information has become available, to be prepared to revise these and perhaps even to revise them somewhat beyond the standard deviation ranges we have computed here since there is the everpresent possibility of systematic error in the data. The only test we (or anyone else) can have indicative of the possible presence of systematic error at any given time, is the compatibility of the equations. Our present 1955 data are not as overdetermined as one could wish (seven equations of observation in four unknowns), and there is not only always the possibility of two or more systematic errors partially or wholly concealing each other; there is also the possibility of systematic error in some of the auxiliary constants for which no test of compatibility may be available. Thus, while we all have faith that there are constants in nature, we must honestly admit that our knowledge of the "best" values of these constants at any one time (i.e., those most representative of the consensus of information at that epoch), is something which requires continual revision, great alertness, and devotion and willingness to make changes on the part of those who take responsibility for distilling these values from the experimental data. Fortunately the situation, as can be seen from Table XVIII, seems to have become more and more stable since about 1941, so that now the fluctuations in the "best" values of any given adjustment, relative to those of the previous adjustment a few years earlier, tend to stay pretty satisfactorily within the range of standard deviation, not only of the previous adjustment but also of all prior adjustments, at least back to 1941. Furthermore, this stability has been accompanied with very great progress in narrowing down the ranges of standard deviation. The next two figures show this history graphically for five well-known constants.

In order to include the wild early fluctuations before 1941, Fig. 18 is plotted to a much coarser scale than the next, a 1 per cent fluctuation being shown by the line segment terminated with arrows.

Fig. 19 shows the fluctuations in the same constants beginning only from 1939 onward, but depicts these to about five times larger scale than Fig. 18 , a 0.1 per cent fluctuation being indicated by the line segment terminated by arrows. The marked improvement in precision and stability with time is fairly evident. 
TABLE XVIII

Progress of Our Knowledge of Six Constants from 1929 to 1955

( $N$ given on physical scale of atomic weights-powers of 10 and units omitted)

\begin{tabular}{|c|c|c|c|c|c|c|c|c|c|c|c|c|}
\hline & & $N$ & & $e$ & & $m$ & & $h$ & & )$^{-1}$ & & $c$ \\
\hline $\begin{array}{l}\text { Birge, } 1929 \\
\text { Birge, } 1932\end{array}$ & 6.066 & \pm 0.006 & $\begin{array}{l}4.770 \\
4.769\end{array}$ & $\begin{array}{l} \pm 0.005 \\
\pm 0.004\end{array}$ & $\begin{array}{l}9.035^{1} \\
9.032\end{array}$ & $\begin{array}{l} \pm 0.010 \\
\pm 0.009\end{array}$ & $\begin{array}{l}6.547 \\
6.5443\end{array}$ & $\begin{array}{l} \pm 0.008 \\
\pm 0.0091\end{array}$ & $\begin{array}{l}137.29 \\
137.307\end{array}$ & $\begin{array}{l} \pm 0.11 \\
\pm 0.048\end{array}$ & $\begin{array}{l}2.99796 \\
2.99796\end{array}$ & $\begin{array}{l} \pm 0.00004 \\
\pm 0.00004\end{array}$ \\
\hline $\begin{array}{c}\text { Dunnington, } \\
1939\end{array}$ & & & 4.8025 & \pm 0.0004 & 1073 & \pm 0.0014 & 21 & \pm & & & 776 & 15 \\
\hline Birge, 1941 & 6.0245 & \pm 0.0011 & 4.8025 & \pm 0.0010 & 9.1066 & \pm 0.0032 & 6.624 & \pm 0.002 & 137.030 & \pm 0.016 & 2.99776 & \pm 0.00004 \\
\hline Birge, 1944 & 6.0250 & $2 \pm 0.00043$ & 4.8021 & \pm 0.0006 & & & & & & & & \\
\hline D \& C, 1947 & 6.0257 & \pm 0.0004 & 4.8024 & \pm 0.0005 & 9.1055 & \pm 0.0012 & 6.6237 & \pm 0.0011 & 137.027 & \pm 0.007 & 2.99776 & \pm 0.00004 \\
\hline$D \& C,{ }^{2} 1950$ & 6.0254 & $4 \pm 0.00011$ & 4.8022 & $3 \pm 0.00007$ & 9.10721 & $1 \pm 0.00025$ & 6.62377 & $7 \pm 0.00018$ & 137.042 & $9 \pm 0.0009$ & 2.997902 & \pm 0.000009 \\
\hline D \& C, 1952 & 6.0247 & $2 \pm 0.00036$ & 4.8028 & $8 \pm 0.00021$ & 9.1085 & \pm 0.0006 & 6.6252 & \pm 0.0005 & 137.037 & $7 \pm 0.0016$ & 2.99792 & \pm 0.000008 \\
\hline D \& C, 1955 & 6.0248 & $6 \pm 0.00014$ & 4.8028 & $6 \pm 0.00009$ & 9.1083 & \pm 0.0003 & 6.62517 & $7 \pm 0.00023$ & 137.037 & $3 \pm 0.0004$ & 2.997930 & \pm 0.000003 \\
\hline
\end{tabular}

1 This is the so-called "spectroscopic" value. The "deflection" value was $8.994 \pm 0.014$. See Part I, Section 2 , for explanation of these terms.

2 The original 1950 values were tabulated with an undetermined " $\Gamma$ correction" attached to each one because the magnitude of the BetheLongmire correction term for finite nuclear size (in the formula for computing $\alpha$ from the hydrogen hyperfine structure measurement of Prodell and Kusch) was not then well known. The subsequent work of Triebwasser, Dayhoff, and Lamb, giving $\alpha$ from their fine structure measurement in deuterium, removed some of the uncertainty and indicated need for a $\Gamma$ correction to the 1950 values of $86.2 \pm 0.9$ ppm. The values tabulated above are the 1950 values after making this correction.

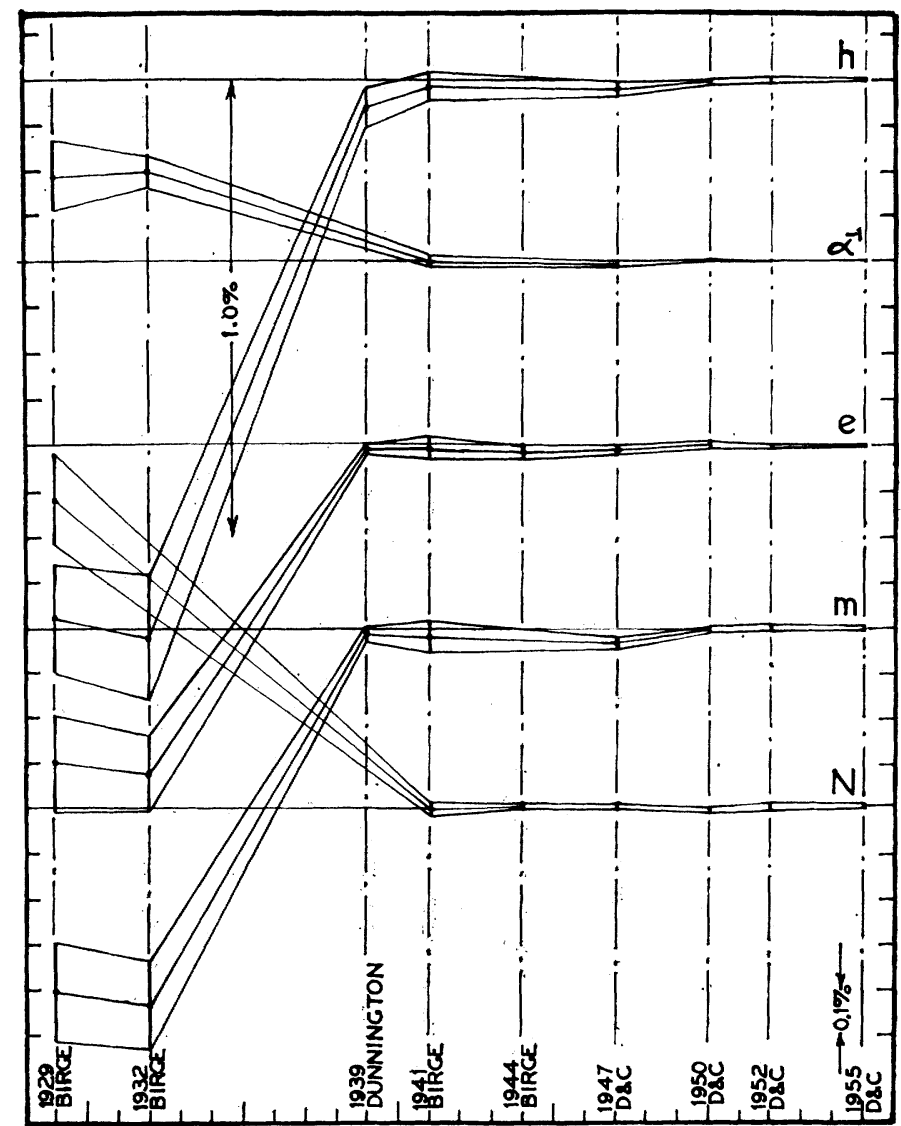

Fig. 18-History of our knowledge of five well-known constants since 1929. A 1 per cent fluctuation is shown by the line segment terminated with arrows.

\section{Discussion of "Weak Spots" Calling for Further Im- provement}

From these figures one might get the impression that we aim to redetermine the constants every three or four years as a matter of routine, just because a certain time has elapsed since the last adjustment. Nothing could be farther from the truth! There is obviously no point in a re-evaluation unless new data have been obtained, or new theoretical ideas developed, or new

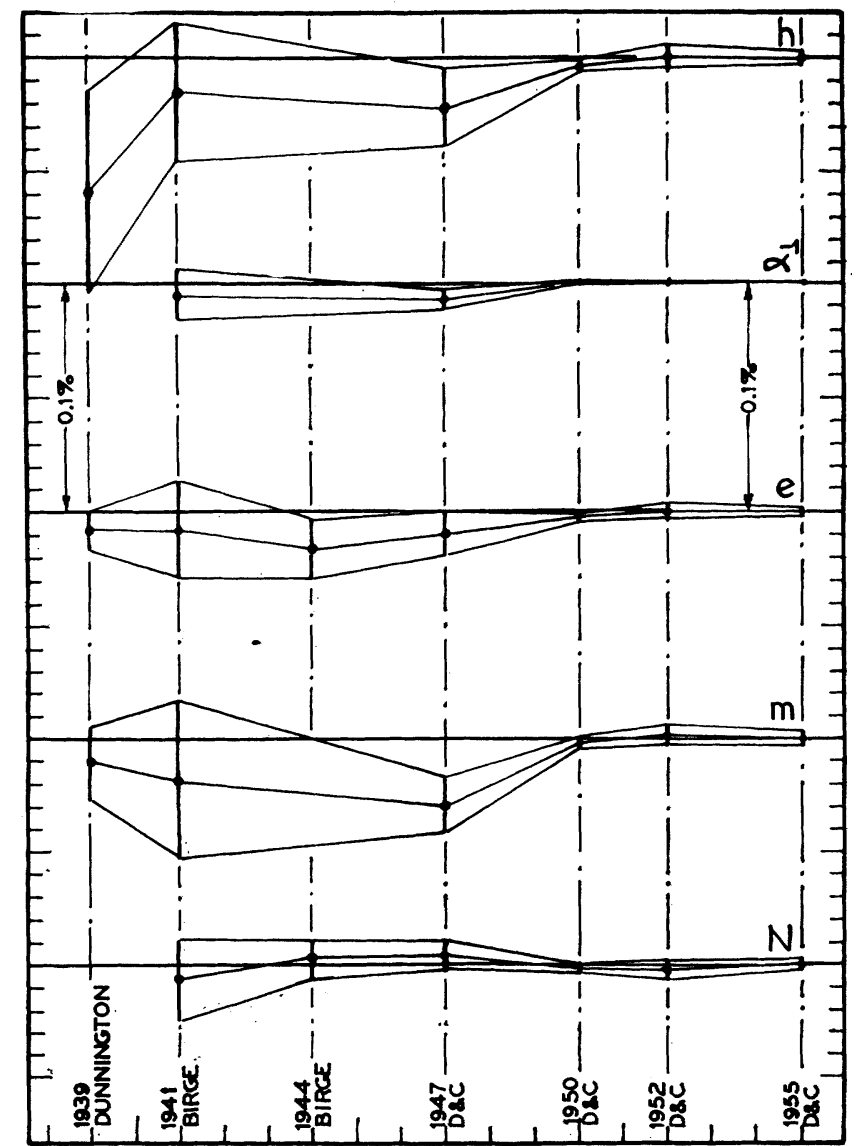

Fig. 19-History of our knowledge of five well-known constants since 1939. The fluctuations are here shown to five times larger scale than in Fig. 18. A 0.1 per cent fluctuation is shown by the line segment terminated with arrows.

sources of systematic error uncovered which call for a revision. Each re-evaluation shown in this figure was called for by at least one and more of ten several important new elements of information entering the picture. This raises the interesting question of when the next adjustment will be called for. Already some small errors of various kinds in the input data of the 1955 adjustment have been either definitely spotted or rendered highly probable, and research work to improve our 
knowledge in these weak spots is under way. With several of these redeterminations still unfinished I feel, however, that it would be premature to make an adjustment now. It seems likely some of the redeterminations now in progress will yield clear answers a year or two from now, and I think we should wait for these. It seems unlikely that any of these improvements will shift our present 1955 values by large amounts compared to their present estimated standard deviations. I shall now list briefly, for your information, these various "weak spots" in the data.

1) An extremely painstaking precision redetermination of the Faraday using the iodine coulometer is under way at the Rockefeller Institute for Medical Research by Dr. MacInnes assisted by Dr. Pray. The iodine coulometer, it is claimed, affords an internal check on the purity of the reaction (as explained in Part I, Section 2) in a more satisfactory fashion than does the silver coulometer. Also, since iodine unlike silver is an isotopically pure substance, it is hoped to avoid some of the uncertainty present with the silver voltameter because of possible variations in isotopic abundance (and hence in mean atomic weight) occasioned in the electrolysis itself. Silver on the contrary has two isotopes in nearly equal abundance, the most favorable proportion for a shift in the mean atomic weight. Electrochemists at the National Bureau of Standards are working on this problem too, I am told, using a perchlorate-silver voltameter, in a new way, by measuring the silver-plated off at the cathode instead of weighing the silver deposited at the anode (since this may contain an indeterminate amount of foreign matter other than silver called "inclusions"). They hope also to be able to obtain a sufficiently precise mass spectroscopic analysis of the silver they use to establish its mean-atomic weight with higher precision than heretofore.

2) The conversion constant, $\lambda_{g} / \lambda_{s}$, from $X$ units to milliangstrom units, which furnishes us with one of our most important data to bridge the gap between our macroscopic scale of lengths and those of atomic magnitudes, is seriously in need of re-examination. The 1940 determination of this constant by Tyrén in Sweden (as explained in Part I, Section 3), took no account of the then unknown Lamb shift in computing Tyrén's calibration spark-line wavelengths, and the details of this work seem now to have been lost so that the correction can no longer be made without repeating the experiment. Kirkpatrick, at the University of Wisconsin, is presently at work on such a repetition, using the 21 -foot concave grating-vacuum spectrometer of Prof. Mack. I am recently informed that Bearden, of Johns Hopkins, also has plans for a redetermination of $\lambda_{g} / \lambda_{s}$, using plane gratings with a crystal monochromator, in order to eliminate the $\alpha_{2}$ component of the $\alpha$ doublet. Better information on this constant is badly needed. Table XIX shows the changes to be expected in eight important output values for each ppm change which may turn out to be required in $\lambda_{g} / \lambda_{s}$.
3) I have already alluded to the discovery of an error in the original theoretical calculation of Karplus and Kroll [43] of the anomaly ratio, $\mu_{e} / \mu_{0}$, of the electron magnetic moment to the Bohr magneton. The recalculation by Petermann [72] has been independently verified by Sommerfield and also by Kroll with complete agreement. This increase of $14.3 \mathrm{ppm}$ in $\mu_{e} / \mu_{0}$ is estimated to produce the following changes in seven of the constants of our 1955 adjustment, if no other modification in that adjustment were made (see Table XX).

4) As already pointed out in Part II, Section 12, work of increased precision by Bergstrand and by Froome [74], [75], subsequent to our 1955 adjustment, has led to still better agreement and an even more precise value of the velocity of light so that this should now probably be taken at $c=299792.7 \pm 0.2 \mathrm{~km} \mathrm{sec}{ }^{-1}$, about $1 \mathrm{ppm}$ lower than we had used. I am told that the National Bureau of Standards also plans further work on this most important of all the constants.

5) The remarkable experimental determination of the hyperfine structure shift in hydrogen by Prodell and Kusch to $0.2 \mathrm{ppm}$ would, when combined with $R_{\infty}$ and $c$, yield the most accurate source of information presently available on the centrally important Sommerfield fine structure constant, $\alpha$, if only we knew how to compute with required precision certain correction terms to take into account the finite extension of the nuclear (proton) charge and magnetic dipole distributions. Some precious information on the "size" of these nuclear extended fields has become available recently from the remarkable high-energy electron scattering experiments at Stanford by Hofstadter, but unfortunately they do not yet seem to measure precisely what the theoreti-

TABLE XIX

Changes (IN PPM) to be Expected in Eight Important Output VALUES For EACH PPM Change IN The InPUT VALUE of $\lambda_{g} / \lambda_{s}$

\begin{tabular}{c|c|c|c|c|c|c|c}
\hline \hline$e$ & $m$ & $h$ & $\alpha$ & $\Lambda$ & $N$ & $F$ & $h c^{2} /(e \Lambda)$ \\
\hline 0.14 & 0.22 & 0.28 & 0.02 & 0.21 & -0.21 & -0.07 & -0.08 \\
\hline
\end{tabular}

(The last item is the voltage-wavelength conversion factor.)

TABLE XX

Changes (in PPM) to Be Expected in Seven Important Output VALUES FOR A 14.3-PPM ChANGE IN THE Electron Magnetic Moment Anomaly Ratio, $\mu_{e} / \mu_{0}$

\begin{tabular}{|c|c|c|c|c|c|c|}
\hline$e$ & $m$ & $h$ & $\alpha$ & $\Lambda$ & $N$ & $F$ \\
\hline-27.0 & -12.2 & -40.0 & -14.0 & -7.6 & 26.3 & -1.0 \\
\hline \multicolumn{7}{|c|}{ Corrected values after making $14.2-\mathrm{ppm}$ change in $\mu_{e} / \mu_{0}$} \\
\hline \multicolumn{7}{|c|}{$\begin{aligned} e & =4.80273 \times 10^{-10} \mathrm{esu} \\
m & =9.1082 \times 10^{-28} \mathrm{~g} \\
h & =6.62491 \times 10^{-27} \mathrm{erg} / \mathrm{sec} \\
\alpha & =7.29719 \times 10^{-3} \\
\alpha^{-1} & =137.0391 \\
\Lambda & =1.002031 \\
N & =6.02502 \times 10^{23}(\mathrm{~g} \text { mole })^{-1}(\text { physical atomic weight) } \\
F & =2.89366 \times 10^{14} \mathrm{esu}(\mathrm{g} \mathrm{mole})^{-1} \text { (physical atomic weight) } \\
F & =9652.18 \mathrm{emu}(\mathrm{g} \text { mole })^{-1}(\text { physical atomic weight) }\end{aligned}$} \\
\hline
\end{tabular}


cians need to know for the above-mentioned correction, in particular the part played by the virtual meson field in perturbing the electric and magnetic interaction between the electron and proton in hydrogen.

This completes my present list of the "weak spots" to which I referred.

\section{Has the Time Arrived to Replace Our Present Arbi- trarily Defined Standards by Some Rational System Based on the Universal Constants?}

When the science of physics was in its infancy, arbitrary units were chosen, such as the kilogram, the meter, and the second. Standards, together with techniques for their reproduction and comparison with other secondary standards, etc., were set up to maintain these units. Although the original motivation was commercial as well as purely scientific, no science of physical measurement could have been developed without this step. One of the important fruits of physical research has been the discovery that nature herself has fundamental units such as the charge on the electron, the rest-mass of the electron, Planck's constant of action, and the speed of light.

The thought lies close at hand that since nature supplies us with such reliable and invariable units in the form of the universal constants, it might be well to abandon the earlier and more arbitrary artificial units completely. This, however, does not seem to me to be advisable, at least for a long time to come. Of primary importance, before all else, in adopting a new fundamental unit or standard of measurement is the precision and reproducibility with which it can be compared with other physical magnitudes of the same dimensionality. Closely-related practical considerations in the choice of a suitable standard are convenience of maintenance with very high stability, ease of making copies to high precision to serve as secondary standards, and ease of forming subunits and multiples with accurately determinable very small and very large ratios to the primary unit or standard. In all this, highest precision is the guiding and all-important principle. The primary unit or standard of a physical quantity must clearly be defined in such a way as to have a precision and repro- ducibility consistent with the most highly developed and precise measuring technique available at the time. The moment physicists learned to compare lengths by means of light waves, with precision and reproducibility superior to the definition of the meter in terms of the two scratches on the meter bar at Sèvres, it became desirable to redefine the meter in terms of wavelengths of some judiciously chosen spectral line. Of course, the standard was redefined so as to be consistent with the old meter, but its precision was sharpened inside the uncertainty limits of the old definition. Similarly, now that we know of spectral lines which we can excite so as to be much more sharply defined and reproducible than the red cadmium line, we desire to abandon the old definition and redefine our unit more sharply still; but, of course, always so that we are not inconsistent with our previous definition. All this seems to me to be such obvious common sense as hardly to require any defense.

Now, as I have just shown, most of the universal physical constants, $e, m, h, c$, etc., together with a multitude of other constant quantities which can be expressed as functions of these, are only known at present in terms of our arbitrarily defined physical units with an accuracy of a few parts in $10^{5}$. Our present arbitrarily defined units are, on the other hand, known and reproducible, with considerably more accuracy than this. The meter, defined in terms of the red cadmium line, is reproducible to a part in $10^{8}$, and the new proposed definition in terms of krypton will improve upon this decidedly.

The second is now defined, as of the October, 1954 Tenth General Conference on Weights and Measures at Paris, France, as the fraction, 1/31,556,925.975, of the tropical year 1900; that is to say, to a precision of order 1 in $10^{10}$. The caesium clock of Essen and Parry at Teddington, England, actually achieves this order of accuracy, maintaining a time standard in terms of the measured frequency for the caesium atom of the central line of the hyperfine Zeeman pattern extrapolated to zero field, namely $\nu_{0}=9,192,631,830 \pm 10 \mathrm{cps}$. The advantage of an atomic time standard over the astronomical one is that the latter only permits the unit of time to be determined after a lapse of several years, if highest

TABLE XXI

Auxiliary Constants

Rydberg wave number for infinite mass

$$
R_{\infty}=109737.309 \pm 0.012 \mathrm{~cm}^{-1}
$$

Rydberg wave numbers for the light nuclei

$$
\begin{aligned}
R_{H} & =109677.576 \pm 0.012 \mathrm{~cm}^{-1} \\
R_{D} & =109707.419 \pm 0.012 \mathrm{~cm}^{-1} \\
R_{\mathrm{He}} 3 & =109717.345 \pm 0.012 \mathrm{~cm}^{-1} \\
R_{\mathrm{He}} 4 & =109722.267 \pm 0.012 \mathrm{~cm}^{-1}
\end{aligned}
$$

Velocity of light

$$
\begin{aligned}
& \text { Atomic mass of neutron (physical scale) } \\
& \qquad n=1.008982 \pm 0.000003 \\
& \text { Atomic mass of hydrogen (physical scale) } \\
& \qquad H=1.008142 \pm 0.000003 \\
& \text { Atomic mass ratio of hydrogen to proton } \\
& H / M_{p}=1.00054461 \text { (Computed using atomic mass of electron } \\
& \qquad \mathrm{Nm}=0.00054875)
\end{aligned}
$$

Atomic mass of the proton (physical scale)

$$
M_{p}=1.007593 \pm 0.000003
$$




\section{TABLE XXI Cont'd}

Atomic mass of deuterium (physical scale)

$$
D=2.014735 \pm 0.000006
$$

Atomic mass ratio of deuterium to deuteron

$$
D / M_{d}=1.00027244 \text { (Computed using atomic mass of electron, }
$$$$
N m=0.00054875 \text { ) (Physical scale) }
$$

Ratio of electron magnetic moment to proton magnetic moment of Koenig, Prodell and Kusch without diamagnetic correction

$$
\left[M_{p} /\left(N m \mu^{\prime}\right)\right]\left(1+\alpha / 2 \pi-2.973 \alpha^{2} / \pi^{2}\right)=658.2288 \pm 0.0004
$$

Correction factor $\mu_{e} / \mu_{0}$ for anomalous magnetic moment of electron

$$
\mu_{e} / \mu_{o}=\left(1+\alpha / 2 \pi-2.973 \alpha^{2} / \pi^{2}\right)=1.0011453
$$

(Computed using the value $1 / \alpha=137.04$ )

Gas constant per mole (physical scale)

$$
R_{0}=(8.31696 \pm 0.00034) \times 10^{7} \mathrm{erg} \mathrm{mole}^{-1} \mathrm{deg}^{-1}
$$

Standard volume of a perfect gas (physical scale)

$V_{0}=22420.7 \pm 0.6 \mathrm{~cm}^{3}$ atmos mole ${ }^{-1}$

TABLE XXII

Least-Squares Adjusted Output Values

(The quantity following each \pm sign is the standard error. Attention is called to the fact that the quantities in this table are "observationally correlated" so that in the computation of the error measures of derived values dependent on two or more of the values in this table the error matrix of Table XV must be used.)

Avogadro's constant (physical scale)

$$
N=(6.02486 \pm 0.00016) \times 10^{23}(\mathrm{~g} \text { mole })^{-1}
$$

Loschmidt's constant (physical scale)

$$
L_{0}=N / V_{0}=(2.68719 \pm 0.00010) \times 10^{19} \mathrm{~cm}^{-3}
$$

Electronic charge

$$
\begin{aligned}
e & =(4.80286 \pm 0.00009) \times 10^{-10} \mathrm{esu} \\
e^{\prime} & =e / c=(1.60206 \pm 0.00003) \times 10^{-20} \mathrm{emu}
\end{aligned}
$$

Electron rest mass

$$
m=(9.1083 \pm 0.0003) \times 10^{-28} \mathrm{~g}
$$

Proton rest mass

$$
m_{p}=M_{p} / N=(1.67239 \pm 0.00004) \times 10^{-24} \mathrm{~g}
$$

Neutron rest mass

$$
m_{n}=n / N=(1.67470 \pm 0.00004) \times 10^{-24} \mathrm{~g}
$$

Planck's constant

$$
\begin{aligned}
h & =(6.62517 \pm 0.00023) \times 10^{-27} \mathrm{erg} \mathrm{sec} \\
\hbar=h / 2 \pi & =(1.05443 \pm 0.00004) \times 10^{-27} \mathrm{erg} \mathrm{sec}
\end{aligned}
$$

Conversion factor from Siegbahn $\mathrm{X}$ units to milliangstroms

$$
\lambda_{g} / \lambda_{s}=1.002039 \pm 0.000014
$$

Faraday constant (physical scale)

$$
\begin{aligned}
F & =N e=(2.89366 \pm 0.00003) \times 10^{14} \mathrm{esu}(\mathrm{g} \text { mole })^{-1} \\
F^{\prime} & =N e / c=(9652.19 \pm 0.11) \mathrm{emu}(\mathrm{g} \text { mole })^{-1}
\end{aligned}
$$

Charge-to-mass ratio of the electron

Ratio $h / e$

$$
e / m=(5.27305 \pm 0.00007) \times 10^{17}{\text { esu } \mathrm{gm}^{-1}}^{-1}
$$$$
e^{\prime} / m=e /(m c)=(1.75890 \pm 0.00002) \times 10^{7} \mathrm{emu} \mathrm{gm}^{-}:
$$

$$
h / e=(1.37942 \pm 0.00002) \times 10^{-17} \mathrm{erg} \mathrm{sec}(\mathrm{esu})^{-1}
$$

Fine structure constant

$$
\begin{aligned}
\alpha=e^{2} /(\hbar c) & =(7.29729 \pm 0.00003) \times 10^{-3} \\
1 / \alpha & =137.0373 \pm 0.0006 \\
\alpha / 2 \pi & =(1.161398 \pm 0.000005) \times 10^{-3} \\
\alpha^{2} & =(5.32504 \pm 0.00005) \times 10^{-5} \\
1-\left(1-\alpha^{2}\right)^{1 / 2} & =(0.266252 \pm 0.000002) \times 10^{-4}
\end{aligned}
$$

Atomic mass of the electron (physical scale)

$$
N m=(5.48763 \pm 0.00006) \times 10^{-4}
$$

Ratio of mass of hydrogen to mass of proton ${ }^{1}$

$$
\begin{aligned}
H / M_{p} & =\left[1-\frac{N m}{H}\left(1-\frac{1}{2} \alpha^{2}\right)\right]^{-1} \\
& =1.000544613 \pm 0.000000006
\end{aligned}
$$

Atomic mass of proton (physical scale)

$$
M_{p}=H-N m=1.007593 \pm 0.000003
$$

Ratio proton mass to electron mass

$$
M_{p} /(N m)=1836.12 \pm 0.02
$$

Reduced mass of electron in hydrogen atom

$$
\mu=m M_{p} / H=(9.1034 \pm 0.0003) \times 10^{-28} \mathrm{~g}
$$

Schrödinger constant for a fixed nucleus

$$
2 m / \hbar^{2}=(1.63836 \pm 0.00007) \times 10^{27} \mathrm{erg}^{-1} \mathrm{~cm}^{-2}
$$

Schrödinger constant for the hydrogen atom

$$
2 \mu / \hbar^{2}=(1.63748 \pm 0.00007) \times 10^{27} \mathrm{erg}^{-1} \mathrm{~cm}^{-2}
$$

First Bohr radius

$$
\begin{aligned}
a_{0} & =\hbar^{2} /\left(m e^{2}\right)=\alpha /\left(4 \pi R_{\infty}\right) \\
& =(5.29172 \pm 0.00002) \times 10^{-9} \mathrm{~cm}
\end{aligned}
$$

Radius of electron orbit in normal $H^{1}$, referred to center of mass

$$
a_{0}{ }^{\prime}=a_{0}\left(1-\alpha^{2}\right)^{1 / 2}=(5.29158 \pm 0.00002) 10^{-9} \mathrm{~cm}
$$

Separation of proton and electron in normal $H^{1}$

$$
a_{0}{ }^{\prime \prime}=a_{0}{ }^{\prime} R_{\infty} / R_{H}=(5.29446 \pm 0.00002) \times 10^{-9} \mathrm{~cm}
$$

Compton wavelength of the electron

$$
\begin{aligned}
\lambda_{c e}=h /(m c)=\alpha^{2} /\left(2 R_{\infty}\right) & =(24.2626 \pm 0.0002) \times 10^{-11} \mathrm{~cm} \\
\chi_{c e}=\lambda_{c e} /(2 \pi) & =(3.86151 \pm 0.00004) \times 10^{-11} \mathrm{~cm}
\end{aligned}
$$

Compton wavelength of the proton

$$
\begin{aligned}
\lambda_{c p}=h /\left(m_{p} c\right) & =(13.2141 \pm 0.0002) \times 10^{-14} \mathrm{~cm} \\
\chi_{c p}=\lambda_{c p} /(2 \pi) & =(2.10308 \pm 0.00003) \times 10^{-14} \mathrm{~cm}
\end{aligned}
$$

Compton wavelength of the neutron

$$
\begin{aligned}
\lambda_{c n}=h /\left(m_{n} c\right) & =(13.1959 \pm 0.0002) \times 10^{-14} \mathrm{~cm} \\
\chi_{c n}=\lambda_{c n} /(2 \pi) & =(2.10019 \pm 0.00003) \times 10^{-14} \mathrm{~cm}
\end{aligned}
$$

Classical electron radius

$$
\begin{aligned}
r_{0} & =e^{2} /\left(m c^{2}\right)=\alpha^{3} /\left(4 \pi R_{\infty}\right) \\
& =(2.81785 \pm 0.00004) \times 10^{-13} \mathrm{~cm} \\
r_{0}{ }^{2} & =(7.94030 \pm 0.00021) \times 10^{-26} \mathrm{~cm}
\end{aligned}
$$

${ }^{1}$ The binding energy of the electron in the hydrogen atom has been included in the quantity. The mass of the electron when found in the hydrogen atom is not $m$, but more correctly $m\left(1-\frac{1}{2} \alpha^{2}+\cdots\right)$. 
TABLE XXII Cont'd

Thomson cross section

$$
(8 / 3) \pi r_{0}^{2}=(6.65205 \pm 0.00018) \times 10^{-25} \mathrm{~cm}^{2}
$$

Fine structure doublet separation in hydrogen

$$
\begin{aligned}
\Delta E_{H} & =(1 / 16) R_{H} \alpha^{2}\left[1+\alpha / \pi+\left(5 / 8-5.946 / \pi^{2}\right) \alpha^{2}\right] \\
& =0.365871 \pm 0.000003 \mathrm{~cm}^{-1} \\
& =10968.56 \pm 0.10 \mathrm{mc} \mathrm{sec}^{-1}
\end{aligned}
$$

Fine structure separation in deuterium

$$
\begin{aligned}
\Delta E_{D}=\Delta E_{H} R_{D} / R_{H} & =0.365970 \pm 0.000003 \mathrm{~cm}^{-1} \\
& =10971.54 \pm 0.10 \mathrm{mc} \mathrm{sec}^{-1}
\end{aligned}
$$

Zeeman displacement per gauss

$$
(e / m c) /(4 \pi c)=(4.66885 \pm 0.00006) \times 10^{-5} \mathrm{~cm}^{-1} \text { gauss }^{-1}
$$

Boltzmann's constant

$$
\begin{aligned}
k=R_{0} / N & =(1.38044+0.00007) \times 10^{-16} \mathrm{erg} \mathrm{deg}^{-1} \\
k & =(8.6167 \pm 0.0004) \times 10^{-5} \mathrm{ev} \mathrm{deg}^{-1} \\
1 / k & =11605.4 \pm 0.5 \mathrm{deg} \mathrm{ev}^{-1}
\end{aligned}
$$

First radiation constant

$$
c_{1}=8 \pi h c=(4.9918 \pm 0.0002) \times 10^{-15} \mathrm{erg} \mathrm{cm}
$$

Second radiation constant

$$
c_{2}=h c / k=1.43880 \pm 0.00007 \mathrm{~cm} \mathrm{deg}
$$

Atomic specific heat constant

$$
c_{2} / c=(4.79931 \pm 0.00023) 10^{-11} \mathrm{sec} \mathrm{deg}
$$

Wien displacement law constant ${ }^{2}$

$$
\lambda_{\max } T=c_{2} /(4.96511423)=0.289782 \pm 0.000013 \mathrm{~cm} \mathrm{deg}
$$

Stefan-Boltzmann constant

$\sigma=\left(\pi^{2} / 60\right)\left(k^{4} / \hbar^{3} c^{2}\right)=(0.56687 \pm 0.00010) \times 10^{-4} \mathrm{ergs} \mathrm{cm}^{-2} \mathrm{deg}^{-4} \mathrm{sec}^{-1}$

Sakur-Tetrode constant (physical scale of atomic weight)

$$
\begin{aligned}
\left(S_{0} / R_{0}\right)_{\mathrm{Ph}} & =\frac{5}{2}+\ln \left\{\left(2 \pi R_{0}\right)^{3 / 2} h^{-3} N^{-4}\right\} \\
& =-5.57324 \pm 0.00007 \\
\left(S_{0}\right)_{\mathrm{Ph}} & =-(46.3524 \pm 0.0014) \times 10^{7} \mathrm{erg} \mathrm{mole}^{-1} \mathrm{deg}^{-1}
\end{aligned}
$$

Sakur-Tetrode constant (chemical scale of atomic weights)

$$
\begin{aligned}
\left(S_{0} / R_{0}\right)_{\mathrm{Ch}} & =-5.57256 \pm 0.00007 \\
\left(S_{0}\right)_{\mathrm{Ch}} & =-(46.3467 \pm 0.0014) \times 10^{7} \mathrm{erg}^{\mathrm{mole}}{ }^{-1} \mathrm{deg}^{-1}
\end{aligned}
$$

Bohr magneton

$\mu_{0}=h e /(4 \pi m c)=\frac{1}{2} e \bar{\lambda}_{c \varepsilon}=(0.92731 \pm 0.00002) \times 10^{-20} \mathrm{erg}_{\text {gauss }^{-1}}$

Anomalous electron moment correction

$$
\left[1+\alpha /(2 \pi)-2.973 \alpha^{2} / \pi^{2}\right]=\mu_{e} / \mu_{0}=1.001145358 \pm 0.000000005
$$

(Computed using adjusted value $\left.\alpha=(7.29729 \pm 0.00003) \times 10^{-13}\right)$

Magnetic moment of the electron

$$
\mu_{e}=(0.92837 \pm 0.00002) \times 10^{-20} \mathrm{erg}_{\text {gauss }^{-1}}
$$

Nuclear magneton

$$
\begin{aligned}
\mu_{n} & =h e /\left(4 \pi m_{p} c\right)=\mu_{0} N m / H^{+} \\
& =(0.505038 \pm 0.000018) \times 10^{-23} \mathrm{erg} \mathrm{gauss}^{-1}
\end{aligned}
$$

Proton moment

$$
\begin{aligned}
\mu & =2.79275 \pm 0.00003 \text { nuclear magnetons } \\
& =(1.41044 \pm 0.00004) 10^{-23} \text { ergs gauss }^{-1}
\end{aligned}
$$

Gyromagnetic ratio of the proton in hydrogen, uncorrected for diamagnetism

$$
\gamma^{\prime}=(2.67523 \pm 0.00004) \times 10^{4} \text { radians sec }{ }^{-1} \text { gauss }^{-1}
$$

2 The numerical constant 4.96511423 is the root of the transcendental equation, $x=5\left(1-e^{-x}\right)$.
Gyromagnetic ratio of the proton (corrected)

$$
\gamma=(2.67530 \pm 0.00004) \times 10^{4} \text { radians } \mathrm{sec}^{-1} \text { gauss }^{-1}
$$

Multiplier of (Curie constant) ${ }^{1 / 2}$ to give magnetic moment per molecule

$$
(3 \mathrm{k} / \mathrm{N})^{1 / 2}=(2.62178 \pm 0.00010) \times 10^{-29}\left(\mathrm{erg} \mathrm{mol} \mathrm{de \gamma ^{-1 }}\right)^{1 / 2}
$$

Mass-energy conversion factors

$$
\begin{aligned}
1 \text { electron mass } & =0.510976 \pm 0.000007 \mathrm{mev} \\
1 \text { atomic mass unit } & =931.141 \pm 0.010 \mathrm{mev} \\
1 \text { proton mass } & =938.211 \pm 0.010 \mathrm{mev} \\
1 \text { neutron mass } & =939.505 \pm 0.010 \mathrm{mev}
\end{aligned}
$$$$
\lg =(5.61000 \pm 0.00011) \times 10^{23} \mathrm{mev}
$$

Quantum energy conversion factors

$$
\begin{aligned}
1 \mathrm{ev} & =(1.60206 \pm 0.00003) \times 10^{-12} \mathrm{erg} \\
E / \tilde{\nu}=h c & =(1.98618 \pm 0.00007) \times 10^{-16} \mathrm{erg} \mathrm{cm} \\
E \lambda_{y} & =(12397.67 \pm 0.22) \times 10^{-8} \mathrm{ev} \mathrm{cm} \\
E \lambda_{s} & =12372.44 \pm 0.16 \mathrm{kv} \times \mathrm{units} \\
E / \nu & =(6.62517 \pm 0.00023) \times 10^{-27} \mathrm{erg} \mathrm{sec} \\
E / \nu & =(4.13541 \pm 0.00007) \times 10^{-15} \mathrm{ev} \mathrm{sec} \\
\tilde{\nu} / E & =(5.03479 \pm 0.00017) \times 10^{15} \mathrm{~cm}^{-1} \mathrm{erg}^{-1} \\
\tilde{\nu} / E & =8066.03 \pm 0.14 \mathrm{~cm}^{-1} \mathrm{ev}^{-1} \\
\nu / E & =(1.50940 \pm 0.00005) \times 10^{26} \mathrm{sec}^{-1} \mathrm{erg}^{-1} \\
\nu / E & =(2.41814 \pm 0.00004) \times 10^{14} \mathrm{sec}^{-1} \mathrm{ev}^{-1}
\end{aligned}
$$

de Broglie wavelengths, $\lambda_{D}$, of elementary particles ${ }^{3}$

Electrons

$$
\begin{aligned}
\lambda_{D e} & =(7.27377 \pm 0.00006) \mathrm{cm}^{2} \mathrm{sec}^{-1} / \mathrm{v} \\
& =(1.552257 \pm 0.000016) \times 10^{-13} \mathrm{~cm}(\mathrm{erg})^{1 / 2} /(E)^{1 / 2} \\
& \left.=(1.226378 \pm 0.000010) \times 10^{-7} \mathrm{~cm} \mathrm{ev}\right)^{1 / 2} /(E)^{1 / 2}
\end{aligned}
$$

Protons

$$
\begin{aligned}
\lambda_{D_{p}} & =(3.96149 \pm 0.00005) \times 10^{-3} \mathrm{~cm}^{2} \mathrm{sec}^{-1 / \mathrm{v}} \\
& =(3.62253 \pm 0.00008) \times 10^{-15} \mathrm{~cm}(\mathrm{erg})^{1 / 2} /(E)^{1 / 2} \\
& =(2.86202 \pm 0.00004) \times 10^{-9} \mathrm{~cm}(\mathrm{ev})^{1 / 2} /(E)^{1 / 2}
\end{aligned}
$$

Neutrons

$$
\begin{aligned}
\lambda_{D n} & =(3.95603 \pm 0.00005) \times 10^{-3} \mathrm{~cm}^{2} \mathrm{sec}^{-1 / v} \\
& \left.=(3.62004 \pm 0.00008) \times 10^{-15} \mathrm{~cm}^{\mathrm{erg}}\right)^{1 / 2} /(E)^{1 / 2} \\
& =(2.86005 \pm 0.00004) \times 10^{-9} \mathrm{~cm}(\mathrm{ev})^{1 / 2} /(E)^{1 / 2}
\end{aligned}
$$

Energy of $2200 \mathrm{~m} / \mathrm{sec}$ neutron

$$
E_{2200}=0.0252973 \pm 0.0000003 \mathrm{ev}
$$

Velocity of $1 / 40 \mathrm{ev}$ neutron

$$
v_{0.025}=2187.036 \pm 0.012 \mathrm{~m} / \mathrm{sec}
$$

The Rydberg and related derived constants

$$
\begin{aligned}
R_{\infty} & =109737.309 \pm 0.012 \mathrm{~cm}^{-1} \\
R_{\infty} c & =(3.289848 \pm 0.000003) \times 10^{15} \mathrm{sec}^{-1} \\
R_{\infty} h c & =(2.17958 \pm 0.00007) \times 10^{-11} \mathrm{ergs} \\
R_{\infty} h c^{2} e^{-1} \times 10^{-8} & =13.60488 \pm 0.00022 \mathrm{ev}
\end{aligned}
$$

Hydrogen ionization potential

$$
\begin{aligned}
I_{0} & =R_{H}\left(h c^{2} / e\right)\left(1+\alpha^{2} / 4+\cdots\right) \times 10^{-8} \\
& =13.59765 \pm 0.00022 \mathrm{ev}
\end{aligned}
$$

${ }^{3}$ These formulas apply only to nonrelativistic velocities. If the velocity of the particle is not negligible compared to the velocity of light, $c$, or the energy not negligible compared to the rest mass energy, we must use $\lambda_{D}=\lambda_{c}[\epsilon(\epsilon+2)]^{-1 / 2}$ where $\lambda_{c}$ is the appropriate Compton wavelength for the particle in question and $\epsilon$ is the kinetic energy measured in units of the particle rest mass. 
TABLE XXIII

Error Elements by External Consistency for 1955 ADJUSTMENT

\begin{tabular}{|c|c|c|c|c|}
\hline \multicolumn{5}{|c|}{ Error matrix, $v_{i j}=\sigma_{i} \sigma_{j} \gamma_{i j}$ (in $\mathrm{ppm}^{2}$ ) } \\
\hline & $\alpha$ & $e$ & $N$ & $\lambda_{g} / \lambda_{s}$ \\
\hline $\begin{array}{l}\alpha \\
e \\
N \\
\lambda_{g} / \lambda_{s}\end{array}$ & $\begin{array}{r}21.52 \\
62.50 \\
-60.60 \\
17.70\end{array}$ & $\begin{array}{r}62.50 \\
373.2 \\
-480.0 \\
139.6\end{array}$ & $\begin{array}{r}-60.60 \\
-480.0 \\
726.0 \\
-210.6\end{array}$ & $\begin{aligned} & 17.70 \\
& 139.6 \\
&-210.6 \\
& 204.2\end{aligned}$ \\
\hline \multicolumn{5}{|c|}{ Standard errors $\sigma_{i}$ (in ppm) } \\
\hline \multicolumn{5}{|c|}{ Correlation coefficients $r_{i j}$} \\
\hline $\begin{array}{l}\alpha \\
e \\
N \\
\lambda_{g} / \lambda_{s}\end{array}$ & $\begin{array}{r}1.000 \\
0.697 \\
-0.485 \\
0.263\end{array}$ & $\begin{array}{r}0.697 \\
1.000 \\
-0.922 \\
0.500\end{array}$ & $\begin{array}{r}-0.485 \\
-0.922 \\
1.000 \\
-0.538\end{array}$ & $\begin{array}{r}0.263 \\
0.500 \\
-0.538 \\
1.000\end{array}$ \\
\hline
\end{tabular}

precision is desired, so that we have our information only in retrospect, while the atomic clock permits standardization to the same precision in a few minutes.

Masses can be compared with the standard, the International Prototype Kilogram, with a precision probably somewhat better than $0.01 \mathrm{ppm}$.

Our electrical standards are now "absolute," that is to say they are defined in terms of the fundamental length, mass, and time standards mentioned above. This difficult feat involved the construction of coils of wire of very accurately established geometrical dimensions to be used either as self-inductances, as mutual inductances, or as elements of precision current balances. The precision in the definition of the electrical units, in this way, is probably better than $\pm 10 \mathrm{ppm}$, and hence is also still superior to the precision of our present knowledge of most of the universal natural constants.

The convenience, accessibility, and superior precision of our existing arbitrarily defined standards, therefore, make their abandonment in favor of the universal natural constants as standards extremely improbable in the foreseeable future. But there are other reasons for hesitating to base a physical system on natural constants even if such important practical considerations were ignored. The truth is we do not as yet know enough to select wisely the natural constants on which to base such a system. In the present state of our understanding of physics there is, in fact, a great embarrassment of choice as regards natural constants that might be adopted as units of measurement. The mass of anyone of a dozen or more so-called fundamental particles might be chosen to replace the kilogram. Which mass is more fundamental, that of an electron, a proton, a neutron, $a \pi^{+}, \pi^{0}$, or $\pi^{-}$meson, etc.? As units of length any one of the famous hierarchy of four present themselves: The Rydberg wavelength, $R_{\infty}{ }^{-1}=4 \pi a_{0} / \alpha$; the Bohr radius, $a_{0}=h^{2} /\left(4 \pi^{2} m e^{2}\right)$; the Compton wavelength, $h /(m c)=2 \pi \alpha a_{0}$; and the Lorentz radius of the electron, $r_{0}=\alpha^{2} a_{0}$. These are lengths characteristic of the electron, but similar definitions for lengths smaller by the factor $1 / 1836$ can readily be written for the proton, and so on, for ali the other so-called fundamental particles! High energy physics may soon reveal an even more fundamental unit of length, the quantum cutoff length. The speed of light could be combined with any one of these fundamental lengths to furnish a fundamental unit of time. Almost the only physical magnitudes about which there would be little doubt as to choice are the unit of charge and the unit of velocity. For these the electronic charge and the velocity of light would certainly be uniquely indicated. Our present arbitarry standards, however, still afford a precision for defining velocity and charge many orders of magnitude superior to the accuracy with which the velocity of light and the electronic charge have been determined.

\section{REFERENCES}

[1] R. T. Birge, Reps. Prog. Phys. (British), vol. 8, p. 90; 1942.

[2] A. O. Nier, Phys. Rev., vol. 77, p. 792; 1950.

[3] E. B. Rosa and G. W. Vinal, Bull. NBS, vol. 13, p. 479; 19161917.

[4] S. J. Bates, Ph.D. dissertation, Univ. of Illinois, Urbana, Ill.; 1912 .

[5] S. J. Bates and G. W. Vinal, J. Am. Chem. Soc., vol. 36, p. 916; 1914.

[6] R. T. Birge, Rev. Mod. Phys., vol. 1, p. 1; 1929.

[7] R. T. Birge, Reps. Prog. Phys. (British), vol. 8, p. 114; 1942.

[8] A. F. Scott, "Electrochemical Constants," NBS Circular 524, Paper No. 1, p. 1; 1953.

[9] D. N. Craig and J. I. Hoffman, "Electrochemical Constants," NBS Circular 524, Paper No. 2, p. 13; 1953.

[10] D. A. MacInnes, "Electrochemical Constants," NBS Circular 524 , discussion following Paper No. 2, p. 20;1953.

[11] M. Siegbahn, "Spectroskopie der Roentgenstrahlen," J. Springer, Berlin, Ger.; 1931

[12] A. H. Compton and S. K. Allison, "X-Rays in Theory and Experiment," D. Van Nostrand Co., Inc., New York, N. Y.; 1934.

[13] E. Ingelstam, "Die K-Spectren der Schweren Elemente," Ph.D. dissertation, Uppsala, Sweden; 1937.

[14] Y. Cauchois and H. Hulubei, "Longueurs d'Onde des Emissiones X et des Discontinuites d'Absorption X," Hermann et Cie, Paris, France; 1947.

[15] W. Bragg, Proc. Roy. Soc. (London), vol. 88A, p. 428; 1913. Vol. 89 A, p. $246 ; 1914$. Vol. 89 A, p. $430 ; 1914$

[16] A. H. Compton, H. N. Beets, and O. K. DeFoe, Phys. Rev., vol. 25 , p. $625 ; 1925$.

[17] E. Bäcklin, Master's thesis, Uppsala, Sweden; 1928.

[18] A. H. Compton and R. L. Doan, Proc. Natl. Acad. Sciences, (U.S.), vol. 11, p. 598; 1926.

[19] J. Thibaud, J. Phys. Rad., vol. 8, p. 13; 1927. Vol. 8, p. 447; 1927.

[20] J. A. Bearden, Phys. Rev., vol. 37, p. 1210; 1931. Vol. 48, p. $385 ; 1935$.

[21] E. Bäcklin, Z. Phys., vol. 93, p. 450; 1935.

[22] M. Söderman, Nature, vol. 135, p. 67; 1935. Ph.D. dissertation, Uppsala, Sweden, 1934.

[23] F. Tyrén, Z. Phys., vol. 109, p. 722; 1938.

[24] R. T. Birge, Am. J. Phys., vol. 13 , p. $69 ; 1945$.

[25] J. A. Bearden, Phys. Rev., vol. 38, p. 2089; 1931.

[26] R. T. Birge, Am. J. Phys., vol. 13, p. 63; 1945.

[27] E. M. Purcell, H. C. Torrey, and R. V. Pound, Phys. Rev., vol. 69 , p. $37 ; 1946$.

N. Bloembergen, E. M. Purcell, and R. V. Pound, Phys. Rev., vol. 73 , p. $679 ; 1948$.

[28] F. Bloch, Phys. Rev., vol. 70, p. 460; 1946.

F. Bloch, W. W. Hansen, and M. Packard, Phys. Rev., vol 70, p. $474 ; 1946$

[29] H. A. Thomas, R. L. Driscoll, and J. A. Hipple, J. Res. NBS, vol. 44, p. 569; 1950. Phys. Rev., vol. 78, p. 787; 1950.

[30] N. F. Ramsey, Phys. Rev., vol. 78, p. 699; 1950.

[31] H. A. Thomas, Phys. Rev., vol. 80, p. 901; 1950.

[32] H. Sommer, H. A. Thomas, and J. A. Hipple, Phys. Rev., vol. 82, p. $697 ; 1951$.

[33] F. Bloch and C. D. Jeffries, Phys. Rev., vol. 80, p. 305; 1950.

[34] C. D. Jeffries, Phys. Rev., vol. 81, p. 1040; 1951.

[35] D. J. Collington, A. N. Dellis, J. H. Sanders, and K. C. Turberfield, Phys. Rev., vol. 99, p. 1622; 1955.

[36] K. R. Trigger, Abstract UA8, Washington, D. C. Meeting, Bull. Am. Phys. Soc., vol. 1, p. 220;1956. 
[37] S. Triebwasser, E. S. Dayhoff, and W. E. Lamb, Jr., Phys. Rev., vol. 89, p. $98 ; 1953$.

E. S. Dayhoff, S. Triebwasser, and W. E. Lamb, Jr., Phys. Rev., vol. 89, p. 106; 1953.

[38] W. E. Lamb, Jr. and R. C. Retherford, (I), Phys. Rev., vol. 79, p. $549 ; 1950$.

[39] W. E. Lamb, Jr. and R. C. Retherford, (II), Phys. Rev., vol. 81, p. $222 ; 1951$.

[40] W. E. Lamb, Jr., (III), Phys. Rev., vol. 85, p. 259; 1952.

[41] W. E. Lamb, Jr. and R. C. Retherford, (IV), Phys. Rev., vol. 86 , p. $1014 ; 1952$.

(Parts I and II of this series by Lamb and co-workers should be consulted for a basic description of the apparatus.)

[42] J. Schwinger, Phys. Rev., vol. 73, p. 416; 1948.

43] R. Karplus and N. M. Kroll, Phys. Rev., vol. 81, p. 73; 1951

[44] W. E. Lamb, Jr., Phys. Rev., vol. 85, p. 263; 1952. [See (134).]

[45] E. R. Cohen, Phys. Rev., vol. 88, p. 353; 1952

[46] J. W. M. DuMond and V. L. Bollman, Phys. Rev., vol. 51, p. $400 ; 1937$.

[47] W. K. H. Panofsky, A. E. S. Green, and J. W. M. DuMond, Phys. Rev., vol. 62, p. 214; 1942 .

[47a] G. Felt, T. Harris, and J. W. M. DuMond, Phys. Rev., vol. 92, p. $1160 ; 1953$.

[48] J. A. Bearden, F. T. Johnson, and H. M. Watts, Phys. Rev., vol. 81 , p. $70 ; 1951$.

[48a] J. A. Bearden and G. Schwarz, Phys. Rev., vol. 79, p. 674; 1950.

[49] A. Nilsson, Arkiv. Fysik, vol. 6, pp. 544-551; 1953.

[50] E. R. Cohen and W. F. Hornyak, Phys. Rev., vol. 72, p. 1127 1947.

A. Tollestrup, W. A. Fowler, and C. C. Lauritsen, Phys. Rev., vol. 78, p. $372 ; 1950$.

C. W. Li, W. Whaling, W. A. Fowler, and C. C. Lauritsen, Phys. Rev., vol. 83, p. 512; 1951.

[51] D. M. Van Patter and W. Whaling, Revs. Mod. Phys., vol. 26, p. $402 ; 1954$.

[52] A. H. Wapstra, Physica, vol. 21, p. 367; 1955.

[53] E. R. Cohen, Phys. Rev., vol. 88, p. 353; 1952.

[54] J. H. Gardner and E. M. Purcell, Phys. Rev., vol. 76, p. 1262; 1949.

[55] J. H. Gardner, Phys. Rev., vol. 83, p. 996; 1951

56] H. Sommer, H. A. Thomas, and J. A. Hipple, Phys. Rev., vol. 82, p. $697 ; 1951$

[57] W. V. Houston, Phys. Rev., vol. 30, p. 608; 1927.

[58] J. W. Drinkwater, O. Richardson, and W. E. Williams, Proc. Roy. Soc. (London), vol. 174, p. 164; 1940.

[59] D. Y. Chu, Phys. Rev., vol. 55, p. $175 ; 1939$

[60] S. H. Koenig, A. G. Prodell, and P. Kusch, Phys. Rev., vol. 88, p. $191 ; 1952$

[61] I. I. Rabi, S. Millman, P. Kusch, and J. R. Zacharias, Phys. Rev., vol. 55 , p. $526 ; 1939$.

[62] R. Beringer and M. A. Heald, Phys. Rev., vol. 95, p. 1474; 1954

[63] A. A. Michelson, F. G. Pease, and F. Pearson, Astrophys. J., vol. 82 , p. $26 ; 1935$.

[64] W. C. Anderson, Rev. Sci. Instr., vol. 8, p. 239; 1937. J. Opt. Soc. Am., vol. 31, p. 187; 1941 .

[65] K. Bol, Master's thesis, Stanford University, Stanford, Calif., 1950. Phys. Rev., vol. 80 , p. $298 ; 1950$.

[66] E. Bergstrand, Nature, vol. 163, p. 338; 1949. Vol. 165, p. 405; 1950. Arkiv. Fysik, vol. 2, p. 119; 1950. Vol. 3, p. 479; 1951.

[67] I. C. C. Mackenzie, Ordnance Survey Professional Papers, No. 19, (Her Majesty's Stationary Office, London, Eng., 1954)

[68] L. Essen, Proc. Roy. Soc. (London), vol. 204 A, p. 260; 1950. Nature, vol. 167 , p. $758 ; 1951$.

[69] K. D. Froome, Proc. Roy. Soc. (London), vol. 213 A, p. 123 ; 1952. Vol. 223 A, p. $195 ; 1954$

[70] L. Essen and K. D. Froome, Nature, vol. 167, p. 512; 1951.
[71] L. Essen and K. D. Froome, Proc. Phys. Soc. B., vol. 64, p. 862; 1951.

[72] A. Petermann, Helv. Phys. Acta, vol. 30, p. 407; 1957.

[73] P. Franken and S. Liebes, Jr., Phys. Rev., vol. 104, p. 1197; 1956.

[74] K. D. Froome, Nature, vol. 181, p. 258; January 25, 1958.

[75] E. Bergstrand, private communication. See also Ann. Française de Chronomet.

[76] J. W. M. DuMond, D. A. Lind, and B. B. Watson, Phys. Rev., vol. 75 , p. $1226 ; 1949$.

D. E. Muller, H. C. Hoyt, D. J. Klein, and J. W. M. DuMond, Phys. Rev, vol. 81, p. 468; 1951.

[77] E. R. Cohen, "Proc. Turin Conf. in honor of A. Avogadro," Nuovo Cim., p. 110; suppl. 1957.

[78] E. R. Cohen, K. M. Crowe, and J. W. M. DuMond, "The Fundamental Constants of Physics," in "Interscience Monograph in Physics and Astronomy, vol. I," Interscience Publishers, New York, N. Y.; 1957.

[79] E. R. Cohen and J. W. M. DuMond, "The Fundamental Constants of Atomic Physics," in "Handbuch in der Physik," S. Flügge, Springer-Verlag, Berlin, Ger., vol. 35; 1957

[80] P. S. Laplace, "Theorie Analytique des Probabilities," 3rd ed., Oeuvres 7, Paris; 1886.

[81] C. F. Gauss, "Theoria Combinationis Observationum Erroribus Minimis Obnoxiae," Werke, Bd. 4, Gottingen, Ger.; 1873.

[82] A. C. Aitken, Proc. Roy. Soc. Edinburgh, vol. 55, p. 42; 1935.

[83] P. S. Dwyer, Ann. Math. Stat., vol. 15, p. 82; 1944.

[84] J. W. M. DuMond and E. R. Cohen, Rep. Natl. Res. Coun. on Atomic Constants; December, 1950.

[85] E. R. Cohen, Phys. Rev., vol. 81, p. 162; 1951.

[86] A. Bjerhammar, Kgl. Tek. Högskol. Handl. No. 49; 1951.

[87] D. C. Brown, Ballistics Research Labs. Rep. 937, Aberdeen, Md.; 1955.

[88] E. Whittaker and G. Robinson, "Calculus of Observations," London, Eng., p. 224; 1944

[89] R. A. Fisher, "Statistical Methods for Research Workers," Edinburgh, Scotland, 1952, (Table 14). These tables have been reproduced in many texts on mathematical statistics.

[90] E. R. Cohen, J. W. M. DuMond, T. W. Layton, and J. S. Rollett, Rev. Mod. Phys., vol. 27, p. 363; 1955.

[91] Spec. Tech. (AEC) Rep. No. 18 [Calif. Inst. Tech. Contract AT (04-3)-63] available on request; gives a full account of the computer program including the complete code and the detailed flow sheet

92] E. R. Cohen, Phys. Rev., vol. 101, p. 1641; 1956.

[93] E. R. Cohen, Proc. Turin Conf, in honor of A. Avogadro, Nuovo Cim., p. 123; suppl. 1, 1957.

[94] A. G. Prodell and P. Kusch, Phys. Rev., vol. 79, p. 1009; 1950.

[95 H. A. Bethe and C. Longmire, Phys. Rev., vol. 75, p. 306; 1949.

[96] J. W. M. DuMond and E. R. Cohen, Rev. Mod. Phys., vol. 25, pp. 698,$705 ; 1953$.

[97] E. R. Cohen and J. W. M. DuMond, "The Fundamental Constants of Atomic Physics," in "Handbuch der Physik," S. Flügge, Springer-Verlag, Berlin, Ger., vol. 55, p. 67; 1957.

[98] R. Hofstadter and R. W. McAllister, Phys. Rev., vol. 98, p. $217 ; 1955$.

[99] R. W. McAllister and R. Hofstadter, Phys. Rev., vol. 102, p. $851 ; 1956$.

[100] E. E. Chambers and R. Hofstadter, Phys. Rev., vol. 103, p. $1454 ; 1956$

[101] H. F. Ehrenburg and R. Hofstadter, Phys. Rev., vol. 110, p. $544 ; 1958$.

[102] M. R. Yearian and R. Hofstadter, Phys. Rev., vol. 110, p. 552; 1958.

[103] R. Hofstadter, Rev. Mod. Phys., vol. 28, p. 214; 1956. (Review article.)

[104] R. Hofstadter, Ann. Revs. Nucl. Sci., vol. 7; 1957. (Review article.) 